\title{
WestVirginiaUniversity
}

THE RESEARCH REPOSITORY @ WVU

Graduate Theses, Dissertations, and Problem Reports

2002

\section{Telecommunications for a deregulated power industry}

Biju Naduvathuparambil

West Virginia University

Follow this and additional works at: https://researchrepository.wvu.edu/etd

\section{Recommended Citation}

Naduvathuparambil, Biju, "Telecommunications for a deregulated power industry" (2002). Graduate Theses, Dissertations, and Problem Reports. 1529.

https://researchrepository.wvu.edu/etd/1529

This Thesis is protected by copyright and/or related rights. It has been brought to you by the The Research Repository @ WVU with permission from the rights-holder(s). You are free to use this Thesis in any way that is permitted by the copyright and related rights legislation that applies to your use. For other uses you must obtain permission from the rights-holder(s) directly, unless additional rights are indicated by a Creative Commons license in the record and/ or on the work itself. This Thesis has been accepted for inclusion in WVU Graduate Theses, Dissertations, and Problem Reports collection by an authorized administrator of The Research Repository @ WVU. For more information, please contact researchrepository@mail.wvu.edu. 


\title{
Telecommunications for a Deregulated Power Industry
}

\author{
by \\ Naduvathuparambil Biju \\ Thesis submitted to the \\ College of Engineering and Mineral Resources \\ at West Virginia University \\ in partial fulfillment of the requirements \\ for the degree of \\ Master of Science \\ in \\ Electrical Engineering
}

Professor Matthew C. Valenti, Ph.D., Chair

Professor Ali Feliachi, Ph.D.

Professor Bojan Cukic, Ph.D.

Lane Department of Computer Science and Electrical Engineering

Morgantown, West Virginia
2002

Keywords: Deregulation, power, wide area measurement systems, phasor measurement units, spread spectrum, orthogonal frequency division multiplexing

Copyright 2002 Naduvathuparambil Biju 


\section{Telecommunications for a Deregulated Power Industry \\ Naduvathuparambil Biju}

(ABSTRACT)

Telecommunication plays a very important role in the effective monitoring and control of the power grid. Deregulation of the US power industry has enabled utilities to explore various communication options and advanced technologies. Utilities are increasingly investing in distributed resources, dynamic real-time monitoring, automated meter reading, and value added services like home energy management sytems and broadband access for its customers.

Telecommunication options like power line communications (PLC) and satellites are fast replacing legacy telephone and microwave systems in the US. Many utilities are showing interest in PLC technology for its use in parametric communications and home broadband access. PLC technology is not new, tracing its roots back to the 1920's and the early days of electrification. However, only until recently, PLC was only capable of transporting just a few bits per second. But all this is changing as communication technology develops. Present day communication techniques like spread spectrum (SS) and orthogonal frequency division multiplexing (OFDM) help to mitigate the hostile nature of the power line channel and enable data transfer rates on the order of a few megabits per second over the power lines. Such high data rates, apart from being used for wide area measurement systems (WAMS), can also be used to provide value added services like broadband access to homes, thereby differentiating utilities in a highly competitive power market by the nature and reliability of services provided.

The objective of this thesis is to study the communication options that are available for utilities today. Phasor measurement units (PMUs) are analyzed in detail and communication delays due to the use of PMUs in wide area measurement systems (WAMS) are also studied. The highlight of this thesis is a close look at the characteristics of the power line channel by presenting a power line channel model and the use of digital modulation techniques like SS and OFDM, which help overcome the effects of such a hostile medium of communication. Various case studies and possible future applications of PLC are also presented. 


\section{Acknowledgments}

This work which highlights the importance of power line communications as a telecommunication alternative would not have been possible without the US DOE/EPSCoR WV State Implementation Award.

Foremost, I would like to thank Dr. Matthew C. Valenti who has been a terrific advisor and under whom I was a research assistant during my duration at the university. His insight, invaluable suggestions, and guidance helped shape this thesis. I would also like to thank Dr. Ali Feliachi, who led the team which was responsible for the DOE project, and Dr. Bojan Cukic, for their suggestions and help. I would also like to thank my colleagues: Jian Sun, Bin Zhao, and Naveen Chandran, at the wireless communications research laboratory (WCRL), for their help.

Finally, on a personal note, I would like to thank my wife Rashmi for all her support and understanding throughout the process and to whom I dedicate this thesis. 


\section{Contents}

Acknowledgments $\quad$ iii

List of Figures vii

List of Tables $\quad$ ix

1 Overview of Deregulated Power Systems 1

1.1 Introduction . . . . . . . . . . . . . . . . . . 1

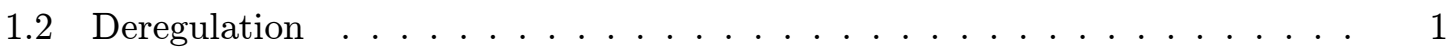

1.2.1 The Need for Deregulation _. . . . . . . . . . . . . . . 1

1.2.2 Deregulation Objectives . . . . . . . . . . . . . 2

1.2.3 Market Structure under Deregulation . . . . . . . . . . . . 3

1.3 Technology at Work for the Future . . . . . . . . . . . . . . . . 4

1.4 Telecommunication Issues . . . . . . . . . . . . . . . . . . . 9

1.5 Theme of the Thesis . . . . . . . . . . . . . . . 11

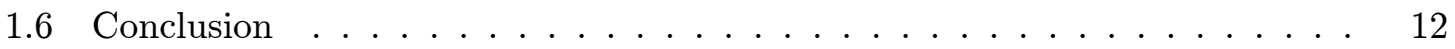

2 Wide Area Measurement Systems 13

2.1 Introduction . . . . . . . . . . . . . . . . . . . . 13

2.2 The Need for Wide Area Measurement Systems . . . . . . . . . . . . . . . 13

2.3 Concept of WAMS . . . . . . . . . . . . . . . . . . 14

2.4 Phasor Measurement Unit . . . . . . . . . . . . . . . . . . . 15

2.4.1 Technology behind PMUs . . . . . . . . . . . . . . . . 15

2.4.2 Technique behind PMU . . . . . . . . . . . . . . . . . . 19

2.4.3 Applications of PMUs . . . . . . . . . . . . . . . . . 21

2.5 IEEE 1344 Synchrophasor Format . . . . . . . . . . . . . . . 22

2.5.1 Message Format . . . . . . . . . . . . . . . . 24

2.5.2 Data Frame . . . . . . . . . . . . . . . . . . . 24

2.5.3 Header Frame . . . . . . . . . . . . . . . . . . 26

2.5.4 Configuration Frame . . . . . . . . . . . . . . . 26

2.5.5 Format of Messages Received by the PMU . . . . . . . . . . . 27

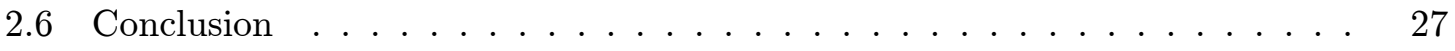


3 Survey of Communication Techniques 28

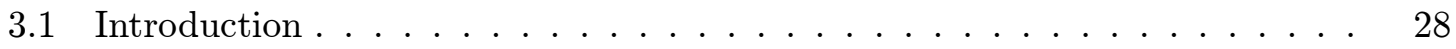

3.2 Fiber-Optic Cables . . . . . . . . . . . . . . . . . . 28

3.2.1 Optical Transmitter . . . . . . . . . . . . . . . . . . 29

$3.2 .2 \quad$ Optical Receivers . . . . . . . . . . . . . . . . . . . . 30

3.2 .3 Channel Capacity . . . . . . . . . . . . . . . . . 30

3.2.4 Fundamentals of Fiber-Optics . . . . . . . . . . . . . . . . . . . . . . . 31

3.2.5 Signal Degradation in Fiber-Optic Cables . . . . . . . . . . . . 34

3.2 .6 Case Studies . . . . . . . . . . . . . . . . . . . 35

3.3 Satellites . . . . . . . . . . . . . . . . . . . . . 35

3.3.1 Design Considerations for Satellites . . . . . . . . . . . . . 36

3.3.2 Satellite Communication Architecture . . . . . . . . . . . . . . . 37

3.3 .3 Case Studies . . . . . . . . . . . . . . . . . . . . . . 39

3.4 Telephone lines . . . . . . . . . . . . . . . . . . . . . . . 39

3.5 Microwave Links . . . . . . . . . . . . . . . . . . . 40

3.6 Power Lines as a Communication Medium . . . . . . . . . . . . . . . 41

3.7 Conclusion . . . . . . . . . . . . . . . . . . 43

4 Communication Delay in WAMS $\quad 44$

4.1 Introduction . . . . . . . . . . . . . . . . . . . 44

4.2 Communication Issues in WAMS . . . . . . . . . . . . . . . . . . 45

4.3 Communication Delays . . . . . . . . . . . . . . . . . . . . . . 47

4.3.1 Communication Delay Causes . . . . . . . . . . . . . . 48

4.3.2 Communication Delay Calculations . . . . . . . . . . . . . 50

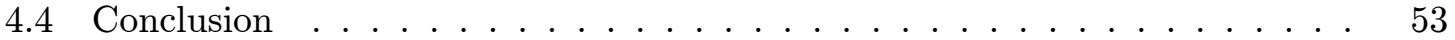

5 Power Line Communications - Overview and Channel Model 54

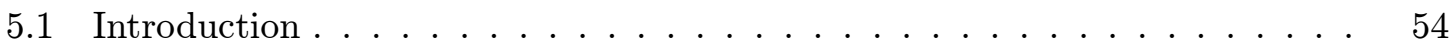

5.2 The Electric Power Supply _. . . . . . . . . . . . . . 54

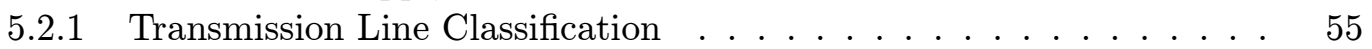

5.2 .2 Transmission Line Modelling . . . . . . . . . . . . . . . . 56

5.3 Concept of Power Line Communications . . . . . . . . . . . . . . . 58

5.4 Characteristics of the Power Line Channel . . . . . . . . . . . . . . . 59

5.4 .1 Attenuation . . . . . . . . . . . . . . 63

5.4 .2 Impulsive Noise . . . . . . . . . . . . . . . . . . . 64

5.4 .3 Impedance Mismatch . . . . . . . . . . . . . . . . . . 64

5.5 Power Line Channel Model . . . . . . . . . . . . . . . . . . . 65

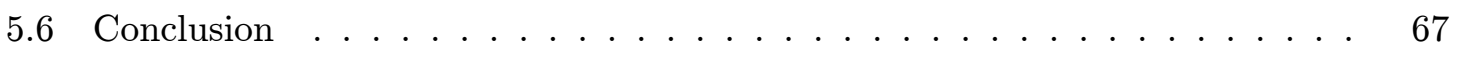

6 Modulation and Coding for PLC $\quad 68$

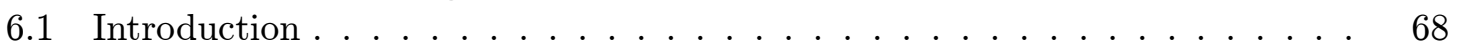

6.2 The Multi-Access Channel . . . . . . . . . . . . . . . . . . 68

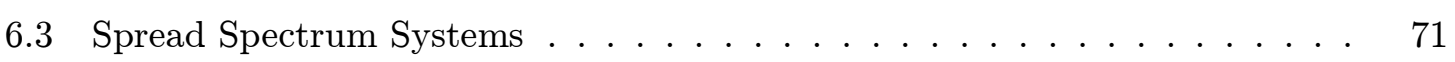

6.3.1 Basic Synchronous CDMA Model . . . . . . . . . . . . . . . 72

6.3.2 Basic Asynchronous CDMA Model . . . . . . . . . . . . . . . 74 
6.3.3 The Spreading Sequence . . . . . . . . . . . . . . 76

6.3 .4 Simulation Results . . . . . . . . . . . . . . . 77

6.4 Rake Receivers . . . . . . . . . . . . . . . . . . . . . 79

6.4.1 Introduction to RAKE Receivers . . . . . . . . . . . . . . 79

6.4.2 Simulation Results . . . . . . . . . . . . . . . . 80

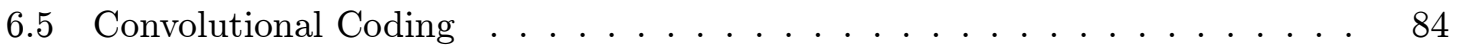

6.5.1 Introduction to Convolutional Coding . . . . . . . . . . . 84

6.5.2 Simulation Results . . . . . . . . . . . . . 84

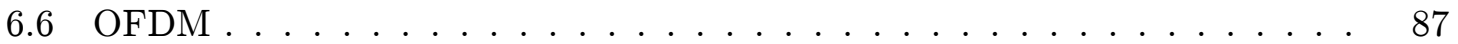

6.6 .1 Introduction to OFDM $\ldots \ldots \ldots \ldots$. . . . . . . 87

6.6 .2 Simulation Results . . . . . . . . . . . . . . . . . 89

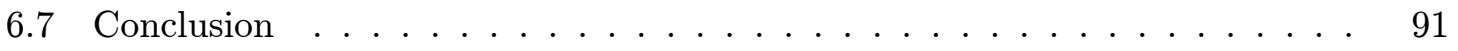

7 Power Line Communication System Design $\quad 92$

7.1 Introduction . . . . . . . . . . . . . . . . . . . . . 92

7.2 PLC System Implementation _ . . . . . . . . . . . . . . . . 92

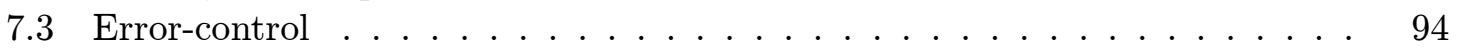

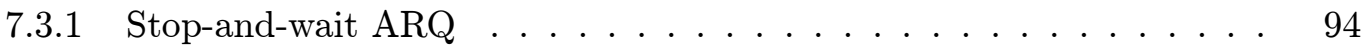

7.3 .2 Go-back-N ARQ . . . . . . . . . . . . . . . . . . 95

7.3.3 Selective-repeat ARQ . . . . . . . . . . . . . . . . 97

7.4 Power Line Communication System Design . . . . . . . . . . . . . . . . . 98

7.4 .1 Protocol Design . . . . . . . . . . . . . . . . . . . . . . . . 98

7.4.2 Access Protocol . . . . . . . . . . . . . . . . . 99

7.4.3 A Combined CSMA/ARQ Protocol . . . . . . . . . . . . 101

7.4 .4 Packet Structure . . . . . . . . . . . . . . . . . . 102

7.5 Throughput Considerations . . . . . . . . . . . . . . 103

7.6 Conclusion ............................ 105

8 PLC System Implementation and Simulations $\quad 106$

8.1 Introduction . . . . . . . . . . . . . . . . 106

8.2 History of PLC . . . . . . . . . . . . . . . 106

8.3 The Power Line Channel . . . . . . . . . . . . . . . . . . . . . . 109

8.4 PLC Simulations . . . . . . . . . . . . . . . . . . . . 110

8.5 Comparison of DS-SS and OFDM . . . . . . . . . . . . 111

8.6 Possible Applications of PLC . . . . . . . . . . . . . . . . . 113

8.7 PLC Case Studies . . . . . . . . . . . . . . . . . . 115

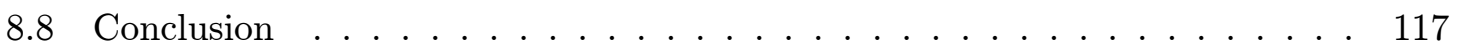

$\begin{array}{ll}\text { A List of Variables } & 118\end{array}$

B List of Acronyms $\quad 121$

$\begin{array}{ll}\text { References } & 125\end{array}$ 


\section{List of Figures}

1.1 A Typical ISO structure . . . . . . . . . . . . . . . . . 3

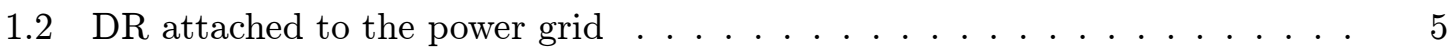

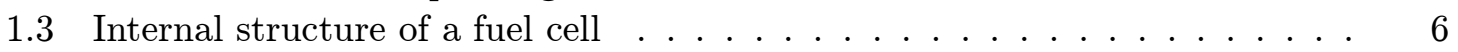

1.4 DC loop Bus . . . . . . . . . . . . . . . . . . 8

1.5 A typical utility substation communication link . . . . . . . . . . 9

2.1 Placement of PMUs in WAMS . . . . . . . . . . . . . . 15

2.2 PMU block diagram . . . . . . . . . . . . . . . . . 16

2.3 Phasor representation of a sinusoid waveform . . . . . . . . . . 20

2.4 Data frame format . . . . . . . . . . . . . . . 25

2.5 Header frame format . . . . . . . . . . . . . . . 26

3.1 Generic optical communication system . . . . . . . . . . . . . . . . 29

3.2 Block diagram of an optical transmitter . . . . . . . . . . . . . . 30

3.3 Block diagram of an optical receiver . . . . . . . . . . . . . . . . . . 31

3.4 Light transmission inside a fiber-optic cable . . . . . . . . . . . . . 32

3.5 Basic satellite communication . . . . . . . . . . . . . . 36

3.6 Satellite communication routing . . . . . . . . . . . . . . 38

3.7 Basic concept of power line communications . . . . . . . . . . . . . . . 42

4.1 General system setup of WAMS . . . . . . . . . . . . . . . . 45

4.2 System overview of PMU implementation by SCE . . . . . . . . . . . . . . . . 48

4.3 Delays in a power system . . . . . . . . . . . . . . 49

5.1 Transmission line characteristics f . . . . . . . . . . . 56

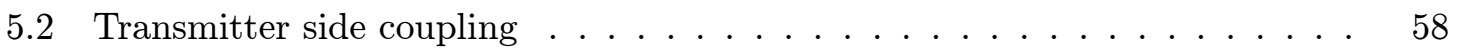

5.3 Receiver side coupling . . . . . . . . . . . . . . . . . . 59

5.4 Multipath propagation . . . . . . . . . . . . . . 62

5.5 Power Line Channel Model . . . . . . . . . . . . . . . . . . . 66

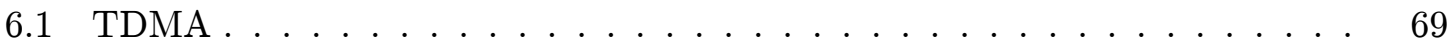

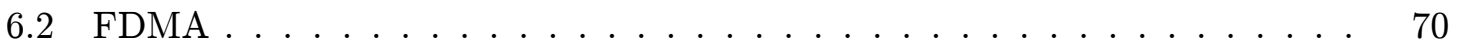

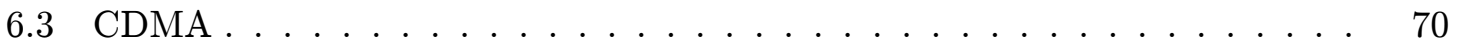

6.4 DS-SS block diagram . . . . . . . . . . . . . . . . . 72 


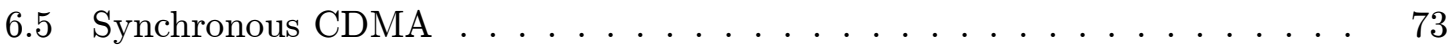

6.6 Synchronous CDMA - receiver end . . . . . . . . . . . . . 73

6.7 Asynchronous CDMA . . . . . . . . . . . . . . . . . 75

6.8 Performance of synchronous and asynchronous CDMA in an AWGN channel, with $\mathrm{G}=7 \ldots \ldots \ldots \ldots \ldots$

6.9 Performance of synchronous and asynchronous CDMA in a flat fading Rayleigh

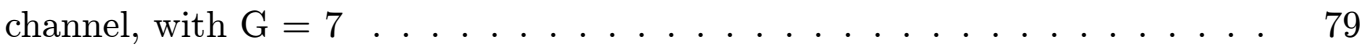

6.10 Multipath propagation, single user, 2 multipath components . . . . . . . 80

6.11 Rake receiver, 2 fingers . . . . . . . . . . . . . . . . . . 81

6.12 Rake receiver performance in comparison with theoretical bounds for single user in Rayleigh fading channel, $\mathrm{G}=7 \ldots \ldots$. . . . . . . . . 83

6.13 Convolutional encoder . . . . . . . . . . . . . . . . 85

6.14 Performance of coded CDMA - convolutional code in Rayleigh fading channel, rate $=\frac{1}{2}, G=7 \ldots \ldots \ldots \ldots \ldots \ldots \ldots \ldots \ldots \ldots \ldots \ldots \ldots \ldots$

6.15 Rake receiver performance - synchronous model - convolutional code, in a Rayleigh fading channel, rate $=\frac{1}{2}, G=7 \ldots \ldots \ldots$

6.16 Rake receiver performance - asynchronous model - convolutional code, in a Rayleigh fading channel, rate $=\frac{1}{2}, G=7 \ldots \ldots \ldots$. . . . . 87

6.17 OFDM system . . . . . . . . . . . . . . . . . 88

6.18 Comparison between uncoded-OFDM - 2 ray model - 8 subcarriers and singleuser BPSK signalling in Rayleigh fading channel . . . . . . . . . . . . . 90

6.19 Convolutionally coded (rate $=\frac{1}{2}, G=7$ ) OFDM versus uncoded BPSK - 2 ray model in Rayleigh fading channel - 8 subcarriers . . . . . . . . . . 90

$7.1 \quad$ PLC system . . . . . . . . . . . . . . . . . . . . . . . . . 93

7.2 Stop-and-wait ARQ timing diagram . . . . . . . . . . . . . . 94

7.3 Go-back-N ARQ timing diagram . . . . . . . . . . . . . . . . . 96

7.4 Selective-repeat ARQ timing diagram . . . . . . . . . . . . . . 97

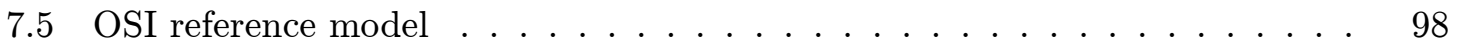

7.6 Block diagram of TDMA transmitter . . . . . . . . . . . . . . . . . 99

7.7 Block diagram of TDMA receiver . . . . . . . . . . . . . . . . 100

7.8 Packet structure for PLC . . . . . . . . . . . . . . . . . . 102

7.9 Throughput versus BER for the combined CSMA/ARQ protocol and for a packet size of 1024 bits . . . . . . . . . . . . . . . 105

8.1 Power Line Channel Model . . . . . . . . . . . . . . . . . . . . . . . . . . 109

8.2 Basic OFDM transmitter . . . . . . . . . . . . . . . . . . . 110

8.3 Basic OFDM receiver . . . . . . . . . . . . . . . . . . 111

8.4 BER performance of uncoded SS without rake receiver, uncoded SS with 2-finger rake receiver, and uncoded OFDM in a PLC channel . . . . . . . . 112

8.5 Sample XML program . . . . . . . . . . . . . . . . . . . 115

$8.6 \mathrm{UCA}$ architecture . . . . . . . . . . . . . . 116 


\section{List of Tables}

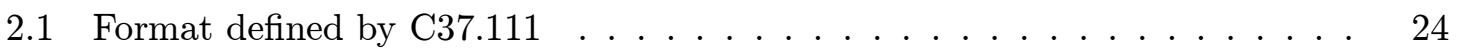

2.2 SMPCNT word definition $\ldots \ldots \ldots \ldots \ldots \ldots \ldots$

4.1 Delay calculations associated with various communication links . . . . . 53

5.1 Transmission line parameters - approximate . . . . . . . . . 57

5.2 Impulse noise classification . . . . . . . . . . . . . . . . . 64 


\section{Chapter 1}

\section{Overview of Deregulated Power Systems}

\section{$1.1 \quad$ Introduction}

Deregulation of the power industry is occurring at a rapid pace. The power market is slowly shifting from a supplier-driven orientation to a consumer-driven orientation. Deregulation promises to bring competition to the forefront, therefore improving power quality and reliability while fastening the pace of technology innovations in the power industry. This chapter provides a dynamic view of what deregulation will bring to the power market in terms of technological advances in power generation and distribution. It will also provide motivation regarding the need for reliable telecommunication systems for an industry which is constantly improvising standards to satisfy the needs of the average consumer. The chapter will conclude by discussing the theme and scope of this thesis.

\subsection{Deregulation}

\subsubsection{The Need for Deregulation}

The North American power grid network is one of the largest and most complex networks in the world. Effective control and monitoring of the grid is crucial to providing reliable power to consumers and industry. However, in recent years, the demand for power has been increasing at a fast pace and the supply is not adequate to meet the ever-growing 
needs of consumers. Traditionally, utilities would add new transmission capacity to handle expected load increases, but because of difficulty in obtaining permits and uncertainty about achieving an adequate rate of return on investment, the total transmission circuit miles are declining while the total demand for transmission resources continues to grow [1]. For example, during the years 1985-1990, 10,000 miles of transmission capacity was added in the US. However, during 1990-1995, only 4,000 miles was added [1].

Today, central station fossil and nuclear plants supply $87 \%$ of the electricity in the United States and most of this electricity is generated by coal, oil, or gas fired plants that are over 30 years old [2]. The reliable operation of such generators is always an impediment to the efficiency required to meet growing demands. The dependence on fossil fuel is tremendous and this needs to be minimized, since fossil fuels will get depleted in the not so far future. The existing distribution systems are also not able to provide value added services like energy management services. Stability of the present power grid structure is another important factor. Under the present scenario, stability of the grid is controlled by localized monitoring stations, which could lead to cascaded outages emerging from local transients in the grid.

\subsubsection{Deregulation Objectives}

As outlined by Electric Power Research Institute (EPRI) and indicated by [1], the objectives of deregulation can be outlined as follows:

- Development of a more efficient, electronically controlled power delivery system to support the quality, security and competitive market requirements of the future.

- Creation of integrated, customer-managed service networks to provide a growing array of customized options and intelligent end-use capabilities.

- Effective management of resources through technology that resolves conflicts between population growth, economic development and resource availability. 


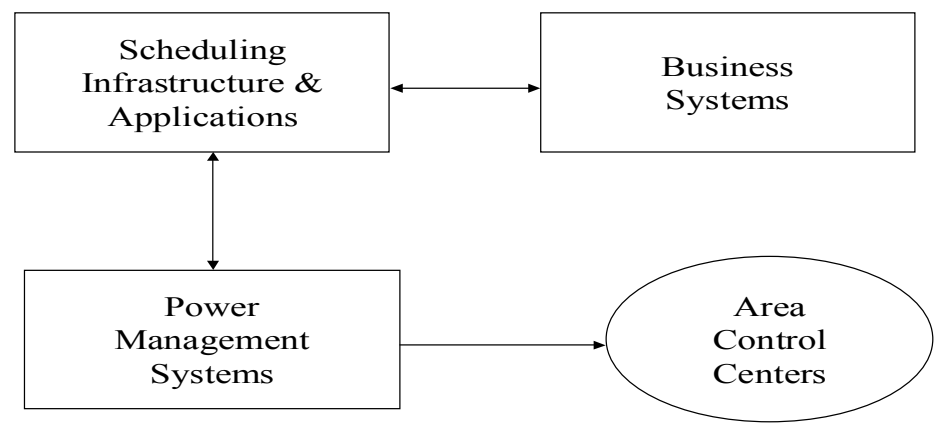

Figure 1.1: A Typical ISO structure

The main objective of deregulation is to provide quality and reliability in power generation and delivery through the use of technology. It is intended to create a competitive oligopolistic market, which would ensure competitive pricing without price caps and usage of advanced technology by the utilities for the generation, distribution, and monitoring of power. Deregulation calls for the creation of a market economy wherein there is complete access to the grid and free flow of information, leading to transparency of operations. To facilitate the entire process, market structures have been put in place which will eventually take over the power grid operations.

\subsubsection{Market Structure under Deregulation}

A typical deregulated market structure can be analyzed by taking the case of the California market structure. The main elements of the California electric market are the independent system operator (ISO), the power exchange (PX) and the scheduling coordinators (SC) [3]. The ISO, shown in Fig. 1.1, is primarily responsible for ensuring the stability of the grid, managing the traffic on the grid, and providing information to all the participants in the market. The PX is primarily responsible for the creation of a competitive spot market for energy, determining hedging rates, and performing settlement procedures for the market. The PX can also act as the SC for its market participants, thereby ensuring that schedules and pricing are adhered to. 
In the ISO block diagram, the scheduling block is responsible for the bidding \& schedule management, grid congestion management, and maintaining balance between demand \& supply. The business system looks after administration matters, invoice generation, and billing statements. The power management system is responsible for maintaining the security and reliability of the power grid. It is in constant communication with the various area control centers that control the stability of the grid. Apart from creating a transparent market environment, deregulation also brings with it new innovations and technological advances, that are presented in the next section.

\subsection{Technology at Work for the Future}

By 2010, a unified, digitally controlled transmission grid will be needed to move large amounts of power precisely and reliably throughout North America and to manage an exponentially growing number of commercial transactions [1]. The use of technology will ensure that power quality is maintained irrespective of the exponential increase in demand. Technological products and services that will be used in the future (some are being currently tested for trial runs) include:

- Distributed resources (DR): This would be one of the biggest outcomes of deregulation. Distributed resources are primarily power generation sources that can be placed at or near customer sites. DR can satisfy customer energy requirements by using them as an on-site energy source and can also provide additional income to customers by way of connecting them to the grid when not required, thereby selling power to the market. Some experts predict that $20 \%$ or more of all new generating capacity built in the United States over the next 10 to 12 years could be for distributed applications, representing a potential market of several tens of gigawatts [2]. Fig. 1.2 shows an example schematic layout of DR when present on the grid.

Some examples of DR include:

1. Micro-turbines: Micro-turbines are basically small gas turbines that burn fuels such as natural gas to produce electricity. Because the low-emission gas turbine 


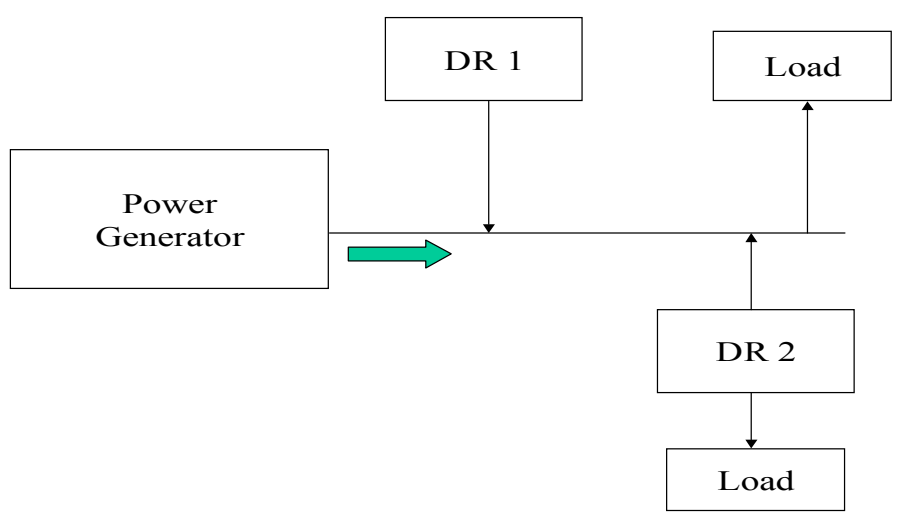

Figure 1.2: DR attached to the power grid

engine has relatively few moving parts, it is quite reliable and can operate continuously for long periods and with little maintenance. In addition, micro-turbines also feature cogeneration capabilities, which means they can harness the heat created by the production of electricity to supply heat for applications such as hot water, enabling facilities to be more cost and energy-efficient. Micro-turbines offer power in the range of $25-100 \mathrm{~kW}$ with efficiencies of about around $30 \%$.

2. Fuel Cells: Fuel cells are one of the most promising options for DR. Although they are an expensive technology, their advantages include high efficiency and reliability. Fig. 1.3 indicates the schematic of a fuel cell.

Pressurized hydrogen fuel is injected into the fuel cell from the anode. As soon as it comes in contact with platinum from the catalyst, the fuel separates into hydrogen ions and electrons. The electrolyte layer does not allow the passage of electrons through it, but it allows the hydrogen protons to pass through. The electrons flow out of the anode into the cathode, which has positive potential, thereby generating electricity (direct current). Oxygen is injected into the fuel cell from the cathode where it eventually breaks up into negative charged ions after coming in contact with the catalyst on the side of the cathode. The negative charged oxygen ions bond with positive charged hydrogen ions, in the process 


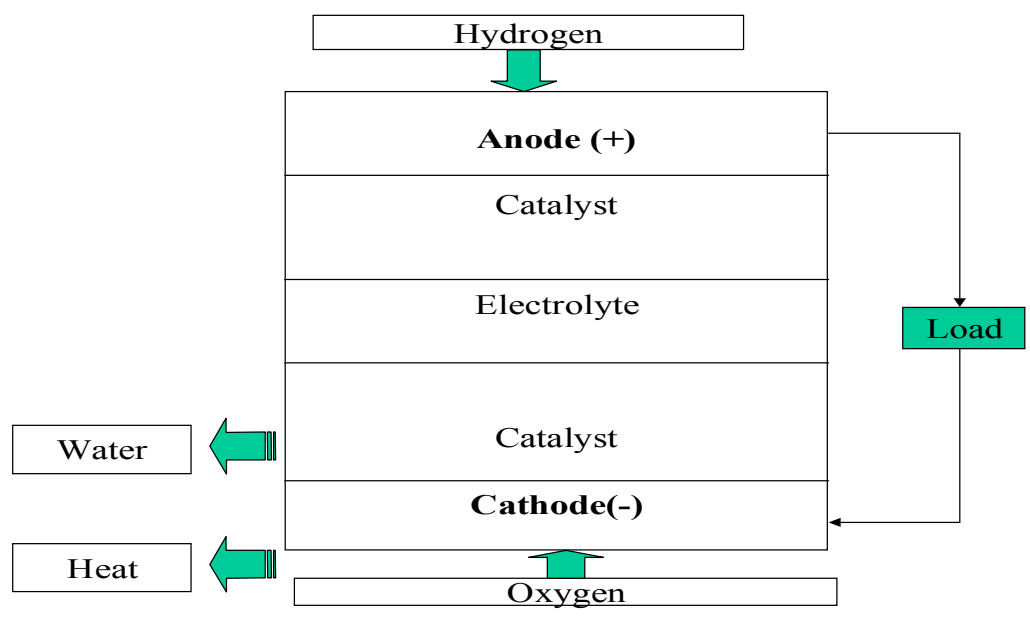

Figure 1.3: Internal structure of a fuel cell

releasing water and heat. Fuel cells come in a wide range of power, from as low as $1 \mathrm{~kW}$ (PEM fuel cells) to as high as $5 \mathrm{MW}$ (molten carbonate fuel cells) [2]. The efficiency of fuel cells ranges from $40 \%$ to $70 \%$. Fuel cells can be used as a distributed utility power, on-site customer power generation, grid-independent power, and for electric vehicles. Compact and modular, fuel cells can be installed almost anywhere, including in most buildings and perhaps someday in mass-market automobiles [4].

3. Photovoltaic cells (PV): These are primarily solar cells that capture heat from the sun and convert it into electricity. Bell Labs originally developed this concept back in the 1950s. PV cells made of crystalline silicon have electrons knocked out of their crystal structure when exposed to sunlight. Impurities added to the silicon make it a semi-conductor thereby preventing electrons from falling back into place. These electrons build up a negative charge in the n-type region, and can only travel back to the positively charged holes left in the p-type region if an external path is created. This flow of electrons creates an electric current in the wire, which may be used directly or stored for later use. PV cells can be stacked to form PV arrays. PV arrays have power ratings in the range of $1 \mathrm{~kW}$ to $1 \mathrm{MW}$. The main problem with PV cells is that they are expensive to produce because of their low efficiency, which is basically around $20 \%$. 
- Advanced Control Technologies: Advanced control technologies like flexible AC transmission system controllers (FACTS) are required to provide almost real-time correction of grid instabilities. Conventional electromechanical controllers are too slow in reacting to transients and this could result in outages if the instability is not rectified almost instantly. FACTS controllers, on the other hand, are based on fast switching thyristors, and can monitor and control the flow of power very effectively. These systems improve grid reliability by counteracting transient disturbances and oscillations almost instantaneously. American Electric Power Company (AEP) is the first utility to install FACTS for real-time grid control.

- Advanced Storage Technologies: Storage of power plays an important role in the future. It ensures presence of power on the grid in the absence of primary generating units. It can also provide a cost-effective solution in the case of photovoltaic cells, which can work only in the presence of solar energy or in the case of wind turbines, which can function only in the presence of wind. Research on super conducting magnetic energy storage is being conducted which could ulimately lead to connecting these advanced technology devices to the power grid. Such devices provide for increased storage capacity and reduced cost of operations towards maintaining storage units.

- Efficient Transmission Lines: Attenuation and loss of power is a major problem during power transmission. In the future, DC loops could carry current because it is easier to carry DC current than AC current since DC current experiences lower resistive losses and no dielectric losses [5]. The costs involved with DC looping would also be lower than AC looping because of the fact that the former requires less insulation. They can also be placed in the same ducts as gas and water pipes because they eliminate the generation of AC induced currents. DC loops may become a common feature in distribution systems by 2010 [5]. Fig. 1.4 indicates a schematic of a DC loop bus and the presence of $\mathrm{AC} / \mathrm{DC}$ converters.

- Automated Metering Systems: Automated metering systems can help utilities 


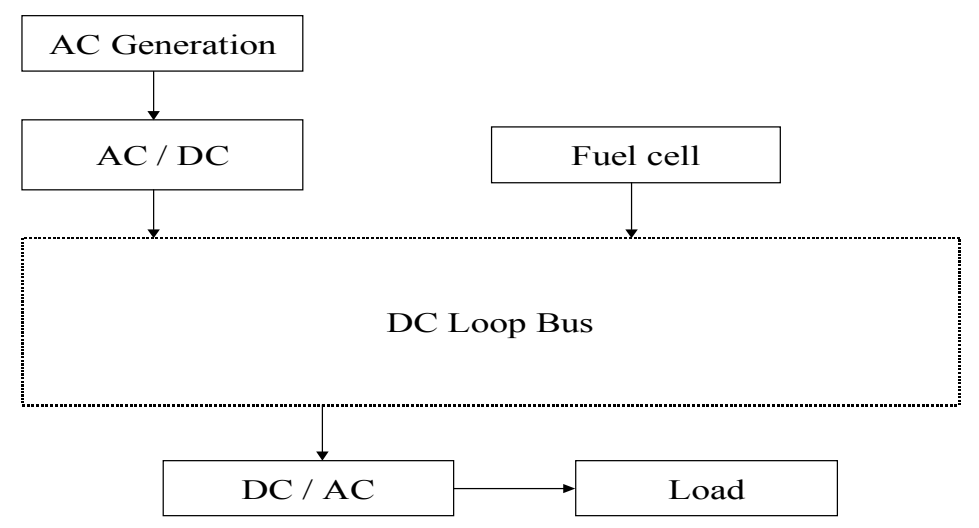

Figure 1.4: DC loop Bus

in providing better and interactive service to the consumers. It will help customers monitor and control their power usage. Many utilities like Southern Company, Pacific Power \& Light, Potomac Electric and Power Company (PEPCO), and Detroit Edison are working with power line communication technology companies like Intellon, Amperion, and Current Technnologies in order to provide automated meter reading (AMR) services to its customers.

- Energy Management Systems (EMS): Energy management systems will play an important role in the future. EMS is basically the ability of the utility customer to control his electricity consumption based on the prevailing electricity rates. EMS interfaces provide information to the customer, information like point-of-time electricity rates and average electricity consumption over a required period of time. Many utilities are increasingly looking at EMS options. Some examples include [6]:

1. Southern California Edison (SCE) is currently experimenting with screen phone interfaces, which will help customers in monitoring their power usage.

2. Boston Edison also plans to incorporate EMS into its portfolio of offerings, with the help of RCN Inc.

3. Pacific Gas and Electric plans to use the Internet towards creating an efficient EMS. 


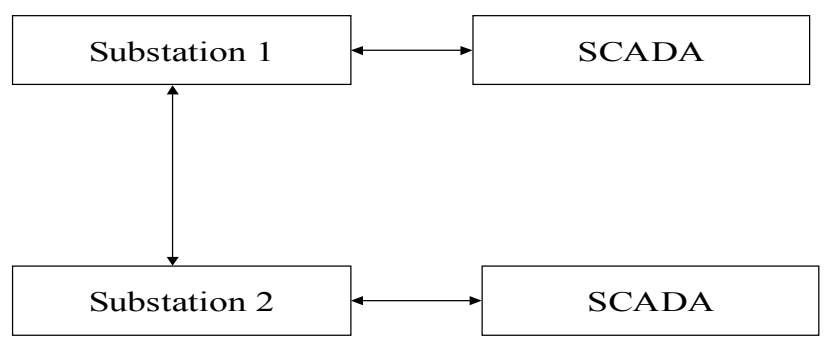

$\longleftrightarrow$ Communication Link

Figure 1.5: A typical utility substation communication link

\subsection{Telecommunication Issues}

Telecommunication plays a very crucial role in integrating systems and ensuring smooth operation by way of exchange of data and information between various systems that are responsible for monitoring and control of the power grid. The purpose of telecommunications for the utilities can be outlined as follows:

- Power System Monitoring: This is the most important function. Data has to be collected from different monitoring nodes (SCADA supervisory control and data acquisition) and has to be sent to the controlling centers, which are responsible for controlling instabilities and transients in the grid. The feasibility of wide-area control applications depends heavily on the realization of standard communication and standardized data modeling of intelligent electronic devices (IEDs) that are responsible for data input to the network. Satellites have been effectively used in monitoring remote locations. Communication networks are crucial for intra-substation and intersubstation communications. Fig. 1.5 presents a schematic of a typical telecommunication network used by the power industry.

- Asset Monitoring: For utilities, it is important to monitor the status of assets, especially in remote areas. A reliable communication link is required to establish the 
correct status and improve the efficiency of operations.

- Remote Monitoring, Diagnostics and Control: It is not always possible to provide manual attention, especially in remote areas that are hard to reach. Telecommunication links can provide up to the minute information from sensors based in remote locations. The data can then be used to remotely control parameters that are not functioning within their stipulated limits.

- Workforce Management: Workforce movement can be monitored and controlled according to the requirements of area control centers.

- Automated Metering and Related Services: Communication links can be established to enable remote meter reading. This will reduce cost of operations and increase efficiency.

- Emergency Communications: In general, it is important to maintain a reliable communication link, especially in case of emergencies. This is also true for utilities, which depend primarily on legacy communication links like telephone lines and have had to switch to satellite based very small aperture terminals (VSATs) when natural calamities like storms throw their telephone-based communication link into disarray.

- Energy Management Services: Utilities can use their communication networks to provide energy management services to customers. Fiber-optic cables and the Internet have been used successfully towards the implementation of EMS. It is beneficial to the customers since it reduces their power usage and it is beneficial to the utilities since it reduces peak time power consumption. Utilities are able to provide better quality of service (QoS) by implementing EMS.

- New Sources of Revenue: Utilities are traditionally afraid to invest in new technology because of the risk of low return on investment (ROI). However, if utilities 
invest in telecommunication infrastructure, they can not only improve their own operations but they can also compete with local phone and cable companies to provide broadband communication alternatives to their customers. This will help in generating additional revenue and ensure better ROI.

\subsection{Theme of the Thesis}

The purpose of this thesis is to provide a detailed inspection of telecommunication issues that arise due to the deregulation of the power industry. It also provides a detailed survey of the use and technical features of phasor measurement units (PMUs) and their integration into wide area measurement systems (WAMS).

Because communication delay is an important aspect governing the performance of WAMS, special consideration is given to the calculation of delay. Large delay in measuring and transmission of crucial data can have a negative impact on control procedures because it might be too late before any action can be initiated to control power grid instabilities. The PMU communicates with control centers using the IEEE 1344 data format and this is independent of the communication link involved during transmission and reception of data. Various communication links can be utilized for the purpose including fiber optics, telephone lines, power lines, satellites, and microwave links. Each communication link has certain advantages and disadvantages associated with it. Certain performance measures for the various links include communication delays involved, economics of setting up and maintaining a particular link, and reliability of the link involved. This thesis looks at various communication links and presents communication delays associated with using each type of a link along with other merits and disadvantages.

The thesis also looks at digital communication using power lines as a medium. It provides an analysis of the power line channel characteristics and suggests a channel model that can be used for power line communications (PLC). The particular modulation techniques of spread spectrum (SS) and orthogonal frequency division multiplexing (OFDM) are presented along with their bit error rate (BER) performance in additive white Gaussian noise (AWGN), fading, and power line channels. The functioning and implementation of 
rake receivers is also presented and it is shown that rake receivers are useful when the information signal undergoes multipath propagation. Just as multipath propagation can take place in a wireless channel due to reflections from surrounding buildings, it can also take place in power line channels due to improper termination of lines leading to fluctuating inductance and capacitance values. Forward error correction (FEC) coding of data is used to mitigate the effects of hostile channels. Coding techniques are presented in this thesis that involve convolutional codes and viterbi decoding. The BER performance of coded data using SS and OFDM modulation techniques are also presented in this thesis.

Apart from the physical layer, the thesis also looks at the upper layers of the open system interconnect (OSI) model and suggests a protocol and system design which can be used for PLC. The protocol design is explained and the advantages and disadvantages of using carrier sense multiple access (CSMA) or time division multiplex access (TDMA) for such a scheme is also discussed.

\subsection{Conclusion}

This chapter discussed the need for, and consequences of, deregulation in the power industry and also highlighted the need for an efficient and reliable communication link for utilities. Future technology was highlighted and the telecommunication needs of the industry were presented. Finally, the theme of the thesis was also presented. 


\section{Chapter 2}

\section{Wide Area Measurement Systems}

\subsection{Introduction}

Local control centers are effective only for controlling local transients or oscillations and they more or less operate independently of each other. The operation of local control centers is not an optimum solution when it comes to dealing with cascaded outages. Wide area protection centers are required to increase the quality and reliability of power. This chapter provides a brief summary of wide area measurement systems (WAMS) and discusses the issue of integrating phasor measurement units (PMUs) into WAMS. The chapter also discusses the IEEE 1344 data format that is used by PMUs to communicate with control stations.

\subsection{The Need for Wide Area Measurement Systems}

The growing trend towards restructuring the power industry and the ever increasing demand for power exchange calls for employing wide area measurement systems (WAMS) for almost real-time measurements that could be used to maintain or improve the stability of the system. Conventional SCADA (supervisory control and data acquisition) / EMS (energy management systems) stability control systems do not provide efficient solutions in the case of cascaded outages as these systems are designed to act locally, based on set operational parameters. These systems do not provide for dynamic coverage of a wide area power network. WAMS, on the other hand, provide for a dynamic coverage of the network and are able to handle cascaded outages through coordinated and optimized 
stabilizing actions [7]. Emerging techniques like phasor measurement units (PMUs) are being used in WAMS, and these complement conventional SCADA / EMS systems thereby providing a wide area system view and increased system stability.

\subsection{Concept of WAMS}

As customers demand new and better quality of services and as the demand for power increases, competing power companies must employ advanced techniques to be able to expand their transmission and distribution capabilities, but at the same time be able to maintain these electric networks with a high level of reliability and security. Apart from the increase in demand for power, WAMS provides for almost real-time fault corrections and ensures power grid stability. In five to ten years, a wide area measurement system that includes an integrated suite of self-adjusting instruments is expected to be available for continuous measurement of all system parameters and monitoring of component status at a high enough sampling rate to be used for practical real time control [8].

In WAMS, PMUs are strategically placed throughout a wide coverage area. The PMUs form part of local devices called system protection terminals (SPT) [7]. SPTs are able to run complete or parts of distributed control algorithms and can communicate directly with other SPTs, substation equipment and system protection centers (SPC), which are responsible for monitoring and control of the power grid. Communication delay plays a crucial role in WAMS by determining the time lag before control action is initiated to dampen power system oscillations.

Fig. 2.1 represents the concept of WAMS. The PMUs measure voltage, current, and frequency phasors using the discrete Fourier transform (DFT) and can detect transients or surges within milliseconds of their occurrence [9]. PMUs use the IEEE 1344 data format for communication with the central monitoring station. In addition to the propagation delay of the particular link, the message format of the PMU and data rate of the link determine the communication delay in the system. Furthermore, there is also a processing delay due primarily to the window size of the DFT. A detailed discussion of communication delays in WAMS is given in chapter 4 of this thesis. 


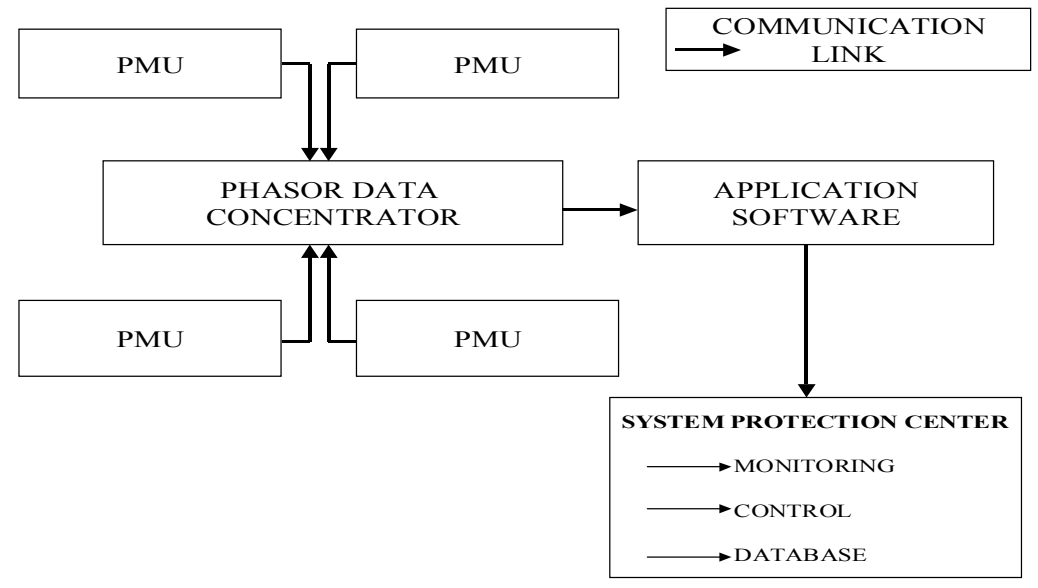

Figure 2.1: Placement of PMUs in WAMS

\subsection{Phasor Measurement Unit}

Phasor measurement units (PMUs) provide for dynamic measurement of power transmission and distribution systems. PMUs also help in implementation of wide-area measurement and control schemes. The primary function of the PMU is to measure phasors (voltage / current / frequency) by way of sampling and processing the analog power signal. Phasor measurements derived from these units could then be relayed to a central processing and control unit with the help of the communication techniques discussed in chapter 3.

\subsubsection{Technology behind PMUs}

PMUs are emerging as the measurement devices of the future. These units measure the voltage, current and frequency phasors at a particular location and can detect transients or surges within milliseconds of their occurrence. The basic technique behind the $\mathrm{PMU}$ is that it converts an analog power signal into discrete samples, from which phasors are constructed using the discrete Fourier transform (DFT). The samples are taken over one cycle or over multiple cycles of the power signal. Data windowing is used to smoothen the frequency spectrum and prevent / reduce overshoot of the signal caused due to discontinuities of the signal in the time domain (Gibbs phenomenon). The use of the DFT in calculating phasors is a better technique than using zero-crossing of the waveforms because zero-crossings could be distorted and could vary due to the presence of harmonics and noise, thus affecting the reliability of the measuring unit. Fig. 2.2 represents the block diagram of 


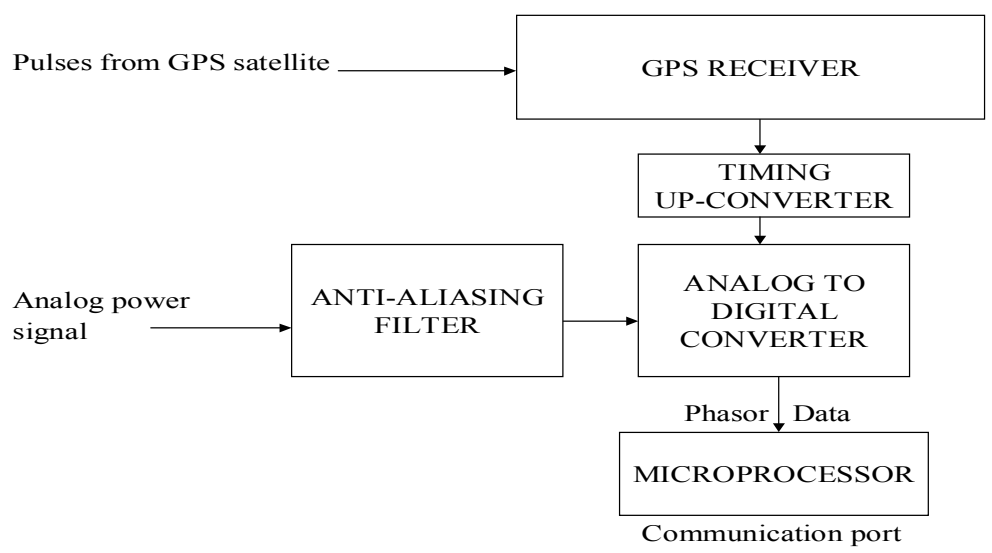

Figure 2.2: PMU block diagram

a phasor measurement unit using the GPS satellite system as a source for synchronization and timing.

Synchronization and time tagging of data is essential to the operation of the PMU. The sources for synchronization pulses needed to sample the analog power signal can be identified as follows [10]:

- AM radio broadcasts: AM radio broadcasts with timing pulses embedded in the signal can be used to obtain synchronization pulses for the PMU. Although AM broadcasts are the most economical sources for synchronization signals, the disadvantages with such type of a system include limited coverage, attenuation of signal, and low accuracy (these systems rarely achieve accuracy better than $1 \mathrm{~ms}$ ) [10].

- Fiber-optic transmission: Fiber-optic transmission offers accuracy in the range of a few microseconds, but these are expensive systems and operate using packet switching digital interfaces, which add further delay to the process.

- Satellite transmission: Satellite transmission is the optimum solution available, at present, in terms of coverage and economy. The systems that are being considered include the GPS satellite system and the INMARSAT satellite system. The global 
positioning system (GPS) is a United States Dept. of Defense satellite based radio and navigation system. It consists of 24 satellites in six orbits at an approximate altitude of 10,000 miles above the earth. The positioning of the orbits and the satellites is such that at least four satellites are in view from locations on the surface of the earth, especially below 60 degrees. The problem of selective availability (SA) with GPS satellites can be rectified by use of the new INMARSAT satellite system, which will be used for synchronizing PMUs and at the same time provide for better accuracy, in the future. INMARSAT satellites have a GPS-like transponder that can be used by slightly modified GPS receivers on earth. SA will not be a problem for INMARSAT as this satellite system is geo-stationary and it will also provide for better accuracy (on the order of 1 microsecond). The one microsecond accuracy has been chosen because it meets all accuracy objectives laid out for power network control (one microsecond corresponds to an angular accuracy of 0.0022 degrees for a $60 \mathrm{~Hz}$ system and 0.018 degrees for a $50 \mathrm{~Hz}$ system).

Currently, PMUs under trial tests are using synchronization pulses from GPS satellite systems. The technique of choice at present is the Navstar GPS satellite transmissions, which transmits one-second pulses, which are then used for the synchronization process. For practical use in the United States, INMARSAT satellite system will be used in the future.

Channels present on the satellites transmit positional coordinates of the satellites from which the location of a receiver station on earth could be identified. Also, as stated earlier, these satellites transmit a one-pulse-per-second signal, along with an identifier for the signal that can be interpreted by the earth-based receivers. In other words, the satellites can not only be used to provide synch pulses but can also be used to provide the physical location of the PMU, the latter playing an important role in tracking and monitoring, during the integration of PMUs into a WAMS scenario.

The GPS receiver not only provides the 1 pulse-per-second (pps) signal but also a time-tag as well. This signal is then divided into the required number of sampling pulses per second by using a phase-locked oscillator. The sampling instant would then be identified as the pulse number within a one-second interval identified by the GPS time-tag. The maximum sampling timing error $E_{t}$, in seconds, is given by [10] 


$$
E_{t}=E_{s}+R_{i} A_{c}
$$

where, $E_{s}$ is the maximum error of the synchronizing signal in seconds, $R_{i}$ is the repetition interval of the synch pulse in seconds and $A_{c}$ is the sampling clock accuracy in seconds / seconds. For example, a GPS-based 1 pps synchronizing signal with an error of 1 sec coupled with a sampling clock with an accuracy of $10^{-6}$ yields a combined sampling error of 2 seconds.

An anti-aliasing filter is used to eliminate aliasing errors in the DFT calculations. 16-bit A/D converters are used to enhance precision in measurement of the analog signals (earlier 12-bit A/D converters were being used). The sampling rate is chosen from the list of Least Common Multiple sample frequencies as defined in the IEEE standard C37-111. The minimum required resolution of the $\mathrm{A} / \mathrm{D}$ converter is given by [10]

$$
R_{\min }=\frac{\operatorname{In} \frac{2 \sqrt{2} F_{s}}{E_{\min }}}{\operatorname{In}(2)}
$$

where $R_{\min }$ is the minimum resolution in bits, $F_{s}$ is the full-scale rating of the signal and $E_{\min }$ is the desired error requirement. 18-bit converters will also be introduced and will eventually replace the existing 16-bit A/D converters, thereby providing an increased level of measurement accuracy.

The exact format for time tagging is defined by the IEEE standard 1344, which is discussed in detail in the latter half of the chapter. The IEEE standard defines the formats for output files provided by the phasor measurement units. Two files (header and configuration) are defined for interpreting the phasor data, as well as the format for the real-time binary output file, which consists of the phasor data and the time-tag. This standard will ensure a standard procedure for accessing PMU data from PMUs that are manufactured by different companies.

The PMU transmits data in the IEEE-1344 format by means of any communication link to a central data processing unit. At present, a majority of the data transmitted for monitoring and control purposes is through telephone lines, by way of modems. These 
PMUs, synchronized by the GPS pulses, form the basis for data collection over a wide area, resulting in effective monitoring and control over a large coverage area the idea behind the implementation of WAMS.

Optimal PMU placement (OPP) is an important aspect of WAMS since it will increase the effectiveness of WAMS [11]. System clustering algorithms are used to ensure proper placements of these PMUs such that effective monitoring of the entire grid can take place with limited number of PMUs.

\subsubsection{Technique behind PMU}

In a PMU, the analog signal is converted into digital data by the process of sampling. For example, if we want to measure the voltage of the signal, then samples are taken for each cycle of the waveform and then using DFT, the fundamental frequency component is extracted from the DFT as follows:

$$
X=\frac{\sqrt{2}}{N} \sum_{k=1}^{N} x_{k} e^{-j k\left(\frac{2 \pi}{N}\right)}
$$

where $N$ is the total number of samples in one period, $X$ is the phasor and $x_{k}$ are the waveform samples. From the equation, we can see that the phasor information is dependent on the number of samples of the waveform and will give a correct representation of the fundamental frequency component even in the presence of transient signals. An error naturally occurs if the measured frequency of the signal is different from that of the nominal frequency and this error is then used as a feedback mechanism to correct the frequency of the signal.

Fig. 2.3 indicates the magnitude and the phase angle of the phasor $\mathrm{X}$ that is derived by taking the discrete Fourier transform of the complex-valued samples (voltage / current). The circle in the graph indicates the region of uncertainty of the phasor. This is due to the presence of frequencies in the signal that are not harmonics of the fundamental frequency and noise in the signal. The source of this noise could be the channel or the electronic equipment related to the power network. The sampling rate should be increased 


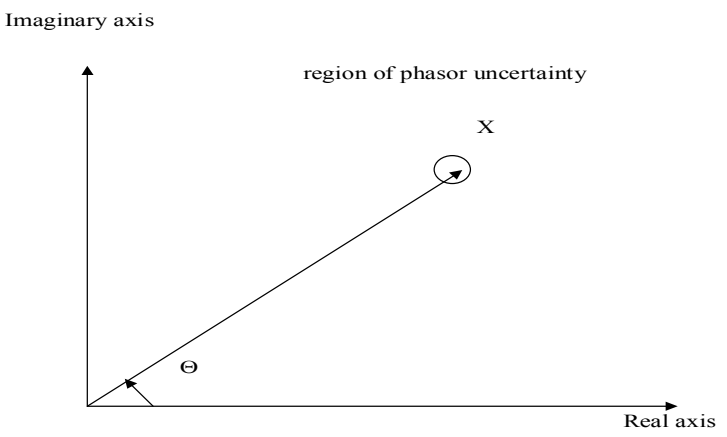

Figure 2.3: Phasor representation of a sinusoid waveform

in the presence of a large area of uncertainty in order to minimize measurement errors. It has been found that the error in estimation is inversely proportional to the square root of the length of the data window.

Equation 2.3 provides for the phasor measurement for one of the phases. Similarly, phasors can be measured for the other two phases of a three-phase supply. The positive sequence phasor can be calculated as follows:

$$
X_{1}=\frac{1}{3}\left(X_{a}+\alpha X_{b}+\alpha^{2} X_{c}\right)
$$

where $\alpha=e^{\frac{j 2 \pi}{3}}$ and $X_{a}, X_{b}$, and $X_{c}$ are the DFT phasor coefficients of each of the three phases. The phasors can be used like "speedometers" to indicate if a part of the system is being overloaded and becoming unstable [12]. The phasor measurement unit derives its inputs from the secondary sides of the three phases of a potential or current transformer and outputs the corresponding positive sequence voltage or current phasor. In the context of power system monitoring and control, positive sequence measurements are used more often than the three phase quantities. The parameters (voltage, current, frequency) are measured over a wide area, at the same instant, using synch pulses obtained from GPS satellite systems. Such a synchronized nature of measurement, over a wide area, provides for locating and correcting transients and imbalances in the grid over a wide region. 


\subsubsection{Applications of PMUs}

The main uses / applications of PMUs include:

- Measuring frequency and magnitude of the phasors: The recursive positive sequence phasor calculation, which is performed with a fixed frequency-sampling clock, produces a complex-valued phasor that has an angular velocity that is equal to the difference between the reference fundamental frequency and the actual frequency of the power signal. If the positive sequence phasor (voltage / current) is

$$
X_{1}=\left|X_{1}\right| e^{j \theta}
$$

then the actual power system frequency is given by

$$
\omega=\omega_{0}+\frac{d \theta}{d t}
$$

where $\omega_{0}$ is the fundamental frequency. The above can be easily understood by an example. When the frequency of the power signal is $61 \mathrm{~Hz}$, the phasor rotates one revolution per second in the counter clockwise direction and if the power signal frequency is $59 \mathrm{~Hz}$, then the phasor rotates one revolution per second in the clockwise direction. This technique is very sensitive and can detect small changes in the frequency of power systems. Frequency deviations of $0.001 \mathrm{~Hz}$ have been measured in the laboratory and in the field [9].

- State estimation: State estimation is the most important part of monitoring a power system. State estimation is the knowledge of all bus voltages and currents at various nodes and branches of a given power network or area. The technology that is presently being used dates back to the 1960s and is based on measurements that are unsynchronized. This results in the inability to dynamically compute the state of the entire system. PMUs can change this scenario, primarily because of the nature of their working. PMUs can relay information on a continuous basis to the data acquisition centers, which can then generate a state vector that will follow the dynamics of the system [13]. With normal dedicated communication circuits operating at 4800 
or 9600 baud, a continuous data stream of one phasor measurement every 2-5 cycles (33.3 $83.33 \mathrm{msec})$ can be sustained [9].

- Instability prediction: Presently, stability analysis of power systems is performed off-line with the help of computer modeling and simulations. Synchronized phasor measurements can enable real-time stability analysis and instability prediction, therefore avoiding the time lag required to recreate the instability scenario on a computer generated model.

- Adaptive relaying of power swings: Out-of-step relays detect power swings on the grid with the help of apparent impedance changes observed by a distant relay [14]. Presently, these out-of-step relays are set based on data observed from stability simulations that are conducted for all reasonable contingencies. The problem with this technique is that the actual conditions may vary as compared to the simulations that were performed for stability testing due to power swings. PMUs can be used to dynamically set the relays thus enabling a better response to power swings [15].

- Improved control and tracking of power systems: Differential protection schemes can be employed using PMU data, thereby minimizing the area of damage by isolating the concerned area. Apart from this, controllers like variable series capacitors (VSC), universal power flow controllers (UPFC), and power system stabilizers regulate the grid based on local feedback, which is then processed by an in-built mathematical model to ensure that the system working meets the objectives laid out. The problem here is that these models may not represent the actual conditions that could exist. Synchronized phasor measurements could provide direct feedback to these controllers, thereby providing for better control over the power system.

\subsection{IEEE 1344 Synchrophasor Format}

Synchronized phasor measurements are forming an integral part of the monitoring and control of the power grid. A significant amount of hardware and software development is taking place in this area and there is a need to integrate the different processes of various 
organizations involved in this field of activity. The IEEE 1344 is a standard that acts as the basis for synchronized phasor measurements [10]. It addresses the following issues:

- Synchronization of data sampling.

- Data to phasor conversions.

- Formats for timing input and phasor data output from the phasor measurement unit (PMU).

The standard not only helps in establishing a basis for phasor measurements, but also facilitates the exchange of data between users who are involved in monitoring and control.

All phasor measurements are synchronized to the universal time co-ordinates (UTC), provided by the US Naval observatory obtained by any of the sources mentioned previously. The signal is basically a 1 pps pulse train where the rising change of the pulse marks the change of second of the UTC time [10]. Phase-locked systems ensure that the receiver locks onto the synchronizing signal. The number of data samples taken per period is an integer and is numbered from 0 to $\mathrm{N}-1$, where 0 is the first sample and coincides with the rising edge of the 1 pps pulse train. The sample rate is chosen as defined by the IEEE standard C37.111 and a few examples are given in Table 2.1. If there is a loss of time / phase lock with the synch pulses, then the PMU will set a flag indicating that the data samples were collected using un-locked data. The synch pulses will then be slewed into alignment at a maximum rate of $70 \mathrm{~s} / \mathrm{s}$. (this corresponds to the fact that synchronization will take place in 2 hours for an offset of $0.5 \mathrm{~s}$ ).

As stated earlier, time-tagging of the data samples and the phasors is done with UTC time. The UTC time format is provided to the PMU in the IRIG-B format, which provides time once per second in seconds through day of the year in a binary coded decimal (BCD) format The BCD is amplitude modulated with a $1 \mathrm{KHz}$ carrier signal. The IRIG-B data format could also be used with modified Manchester coding with a clocking rate of 1 
Table 2.1: Format defined by C37.111

\begin{tabular}{|c||c||c|}
\hline Samples/Cycle & Number of Samples-60Hz & Number of Samples-50Hz \\
\hline 4 & 240 & 200 \\
\hline 6 & 360 & 300 \\
\hline 8 & 480 & 400 \\
\hline 12 & 720 & 600 \\
\hline 16 & 960 & 800 \\
\hline 24 & 1440 & 1200 \\
\hline
\end{tabular}

$\mathrm{kHz}$ with the rising edge indicating a binary 1 and the falling edge indicating a binary 0 (high precision time code format). The data edges are in phase with UTC and this digital format eliminates the need for a separate 1 pps synchronization. This method also has the advantage of being integrated with cable or fiber-optic systems easily.

The time tag (4 Bytes (B)) of the data samples indicates the second of the century (SOC measured from midnight of January 1, 1900) the data sample was measured. The time tag of the phasor data has two parts. The first part is the SOC (4B) the same as the time tag of the data sample and the second part (2B) represents the sample number of the last sample used in the phasor calculation.

\subsubsection{Message Format}

The PMU transmits messages in three types of frames data frame, header frame and configuration frame. The first 4 bytes of each frame are the SOC time mark, which provides a useful combination for frame synchronization. This is followed by the sample count (SMPCNT) word (2 B) that determines the type of the frame and is defined by table 2.2. The three most significant bits of the SMPCNT word identify the message type. Apart from this, every frame is terminated by a cyclic redundancy check (CRC) code to validate the data in the frame. The CRC uses the generator polynomial $D^{16}+D^{12}+D^{5}+1$.

\subsubsection{Data Frame}

The data frame provides information regarding phasor data and the state of digital inputs on each channel. It also defines the trigger status of frequency, angle, over-current, 
Table 2.2: SMPCNT word definition

\begin{tabular}{|l||l||l|}
\hline Bits 15-14-13 & Frame Type & Definitions \\
\hline 000 & Data frame & Bits 0-12: sample count \\
\hline 001 & Header frame & Bit 12: first frame flag \\
\hline & & Bit 11: last frame flag \\
\hline & & Bits 0-10: frame count for file \\
\hline 010 & Configuration frame & Bit 12: first frame flag \\
\hline & & Bit 11: last frame flag \\
\hline & & Bits 0-10: frame count for file \\
\hline $011-100$ & Reserved & Bits 0-12: to be assigned \\
\hline $101-111$ & User-defined & Bits 0-12: user defined \\
\hline
\end{tabular}

First

transmitted

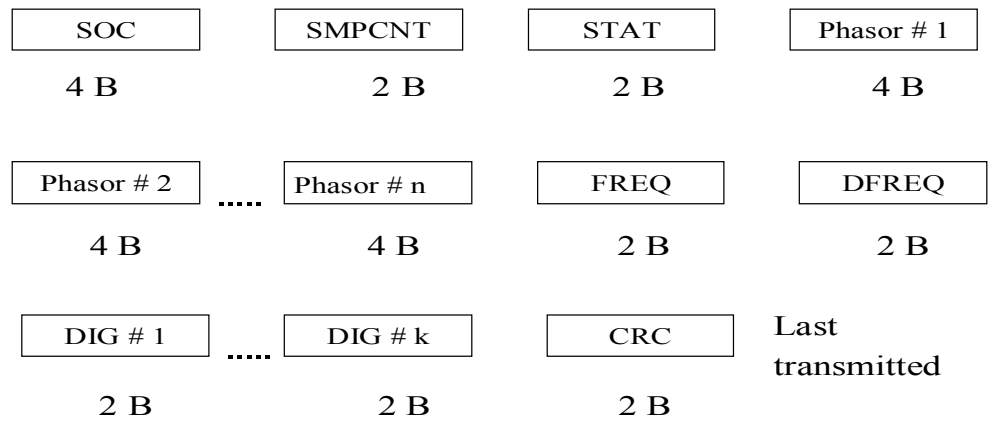

Figure 2.4: Data frame format

under-voltage, and rate of frequency change. The format of the data frame is given by Fig. 2.4 .

The SOC is the second of the century as described earlier and has a length of 4 bytes. The SMPCNT, with a length of 2 bytes, identifies the type of message and keeps a count of the frame. The status word STAT, with a length of 2 bytes, defines the validity of the data and the validity of the synchronization status. It also defines the trigger status of frequency, angle, over-current, under-voltage and rate of frequency change. Each Phasor data has a length of 4 bytes, 2 bytes for the real part and 2 bytes for the imaginary part of the phasor. The frequency (FREQ) defines the deviation of the frequency from the nominal value. DFREQ defines the rate of change of the frequency. DIG, digital channel, data 


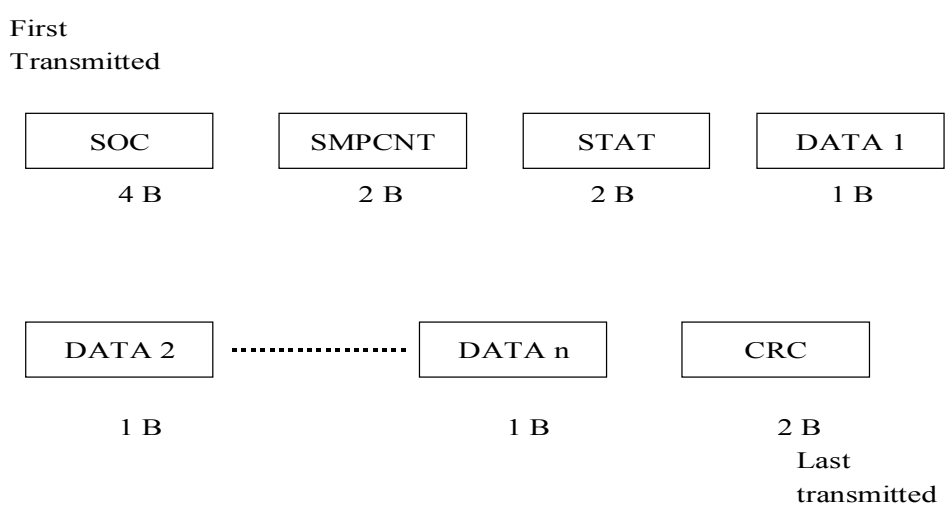

Figure 2.5: Header frame format

defines the state of the digital inputs on each channel that the data is being sent.

\subsubsection{Header Frame}

The header frame is an ASCII file that contains information about the PMU, the data source, algorithms, and the analog filters used. This frame has the same SOC, SMPCNT, STAT and CRC as all the other frames, but apart from this, it does not have a fixed format. The header frame format is specified by Fig. 2.5

\subsubsection{Configuration Frame}

This is a machine-readable binary file, which contains the following information:

- Station name (16 B) and PMU identification code (8 B).

- Number of phasors (2 B).

- Number of digital channels (2 B).

- Channel and phasor names (16 B for each phasor and each digital channel). 
- Nominal line frequency $(50 / 60 \mathrm{~Hz}),.(1 \mathrm{~B})$.

- Transmission period of the phasors $(2 \mathrm{~B})$ in terms of the number of fundamental frequencies.

\subsubsection{Format of Messages Received by the PMU}

The PMU also receives messages which indicate when to start transmitting data / files and when to stop transmitting data / files. The received data format has a 4 byte SOC, 8 byte IDCODE that is used to verify the message by matching it with a code number stored in the PMU. This is followed by the 2 byte command code, which initiates action from the PMU. The last 2 bytes are made up of the CRC code.

\subsection{Conclusion}

WAMS is an important concept and represents an important technological leap in measurement and control technology. WAMS allows efficient and dynamic control of the power grid in almost real-time and helps prevent cascaded outages. This chapter presented an overview of WAMS and the integration of PMUs into WAMS. It also discussed the data formats that are used by PMUs to communicate with the control centers. 


\section{Chapter 3}

\section{Survey of Communication Techniques}

\section{$3.1 \quad$ Introduction}

Various communication links are used by utilities for communication purposes. These links include fiber-optics, satellites, telephone lines, microwave links, and power lines. The decision to use a particular type of communication link is based on the effectiveness of the link in doing the job, the communication delays that the link adds to the system, and the infrastructure costs involved in setting up a particular communication link. The links can not only be used for communication purposes by the utilities, but also as a means of revenue generation by providing broadband access to its customers.

\subsection{Fiber-Optic Cables}

Fiber-optic transmission systems have many advantages over copper wire transmission systems. Fiber-optic cables have lower transmission losses and wider bandwidths as compared to copper wires. They are light, flexible and easy to install compared to copper cables, which are bulky. This is an important factor when it comes to the installation of communication links in crowded underground metropolitan ducts. Other advantages of fiber-optic cables include immunity to electromagnetic interference and very low fiber-tofiber cross talk, which results in high data security. A generic optical communication system is presented in Fig. 3.1. 


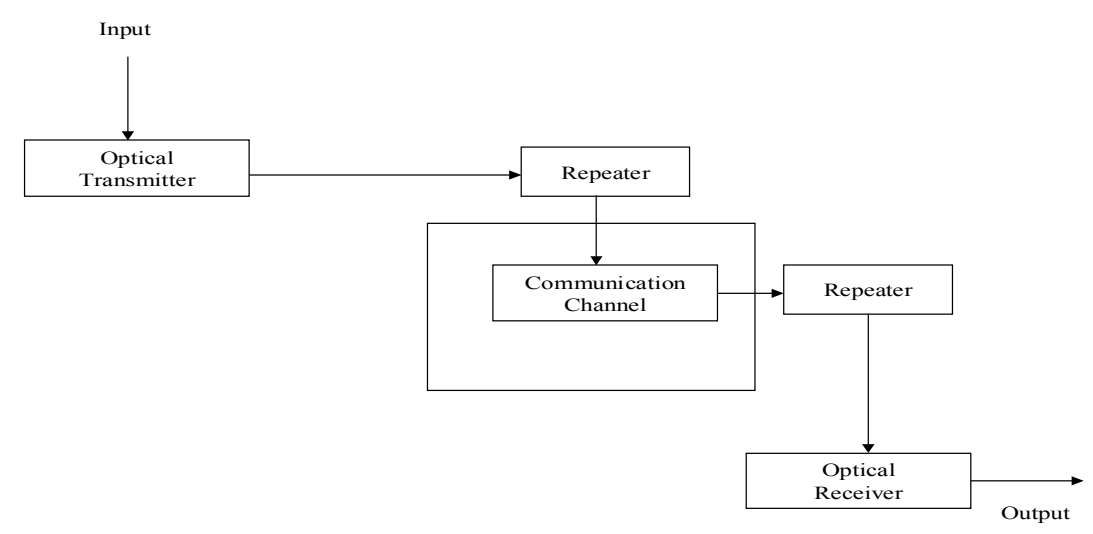

Figure 3.1: Generic optical communication system

The generic model consists of three blocks transmitter, channel and receiver which is common to any communication link. Optical communication systems can be broadly classified as guided transmission systems in which the optical beam is spatially confined by using fiber-optic cables, and unguided transmission systems in which the optical beam propagates through space similar to the propagation of radio waves. The latter requires accurate pointing between the transmitter and the receiver and is used for inter-satellite communications.

\subsubsection{Optical Transmitter}

The optical transmitter converts the electric signal into an optical signal and injects it into the fiber. Fig. 3.2 represents a typical optical transmitter. The optical source is a light emitting diode (LED) or a semiconductor laser, although the latter is more widely used because the semiconductor laser is much more powerful than an LED in terms of light emission and a laser is also relatively easier to modulate. External modulators could be used to modulate the beam, but these are rarely used since the output of a semiconductor source can be modulated directly by varying the injection current. The coupler is a micro-lens that focuses the optical signal into the optical fiber. Optical transmitters can be made to operate up to about $1015 \mathrm{~Gb} / \mathrm{sec}$ [16]. 


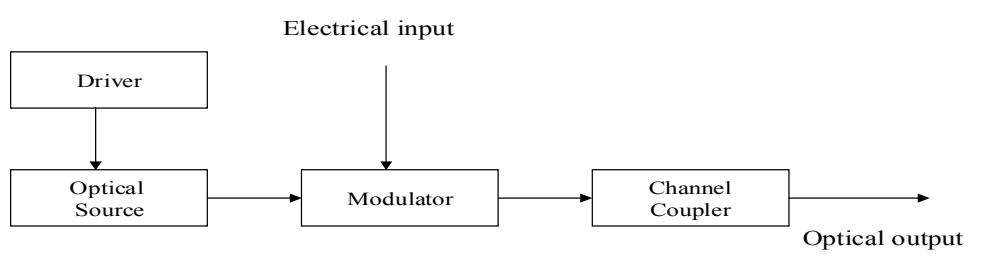

Figure 3.2: Block diagram of an optical transmitter

\subsubsection{Optical Receivers}

Fig. 3.3 represents an optical receiver. The optical receiver converts the optical beam into an electrical signal, from which the data bit is interpreted. The coupler focuses the optical beam onto a photo-detector. The demodulator determines the bit logic. The performance of an optical communication system is characterized by its bit error ratio (BER).

The receiver sensitivity also plays an important role in determining the BER. For any receiver, the process of photo-detection itself introduces some amount of noise. This is referred to as quantum noise or shot noise, and is primarily due to the particle nature of the electrons in the photo-detector circuit. The receiver electronics can also result in thermal noise and amplifier noise as well. Hence, the receiver sensitivity is degraded by all possible noise elements, which are directly related to the bit rate, and therefore limits the rate at which the channel can transport data.

\subsubsection{Channel Capacity}

The channel capacity of a noisy communication channel is defined as

$$
C=B \log _{2}\left(1+\frac{S}{N}\right)
$$




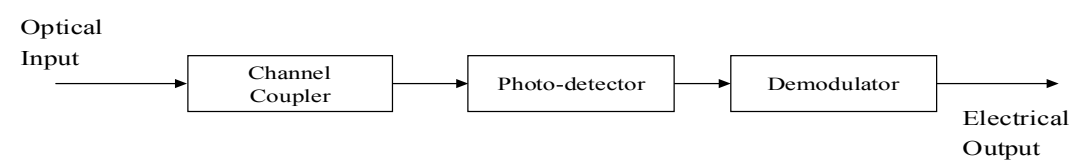

Figure 3.3: Block diagram of an optical receiver

where $C$ is the channel capacity in bits/sec, $B$ is the bandwidth of the channel in $\mathrm{Hz}, S$ is the average signal power, and $N$ is the average noise power. Channel capacity cannot be increased indefinitely by limitlessly increasing the bandwidth offered by the channel. This is due to the fact that shot noise increases linearly with bandwidth. Hence by using

$$
N=N_{o} B
$$

where $N_{o}$ is the spectral density of shot noise and by taking the limits of infinite bandwidth, the channel capacity is limited by $[16]$

$$
C \leq C_{\max }=\left(\frac{S}{N_{o}}\right) \log _{2} e
$$

The channel capacity for optical communication systems is much higher than that of conventional copper cable systems, on the order of tens of Gigabits/second.

\subsubsection{Fundamentals of Fiber-Optics}

To understand the concept of fiber-optic transmission, it is important to understand Snells law, which indicates the transmitted ray direction, and is given by

$$
\frac{\sin \theta_{t}}{\sin \theta_{i}}=\frac{n_{1}}{n_{2}}
$$




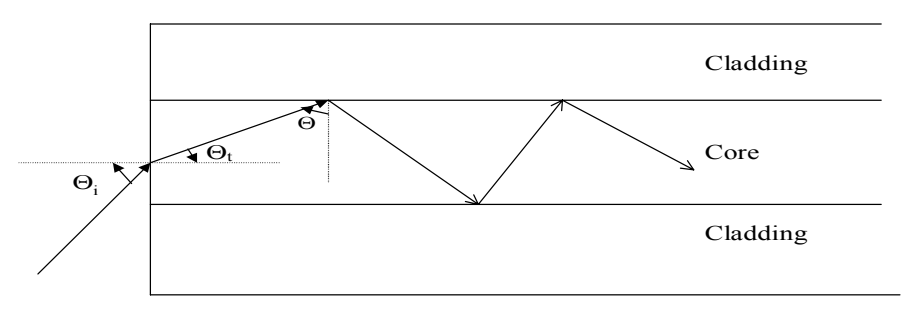

Figure 3.4: Light transmission inside a fiber-optic cable

where $\theta_{t}$ is the angle of transmission, $\theta_{i}$ is the angle of incidence, and $n_{1}$ and $n_{2}$ are the refractive indices of the incident and transmission regions respectively (core and cladding). The equation indicates that the transmitted ray is bent towards the normal when traveling from a medium having a low refractive index into a medium with a higher refractive index. Fig. 3.4 shows an example of light transmission inside a fiber-optic cable.

The light rays are trapped inside within the core of the fiber-optic cable since the refractive index of the core $n_{1}$ is greater than the refractive index of the cladding $n_{2}$. The critical angle is given by

$$
\sin \theta_{c}=\frac{n_{1}}{n_{2}}
$$

The incident angle must be greater than the critical angle for total internal reflection so as to allow the propagation of light rays in the core of the cable. To obtain total reflections, the boundaries must be smooth; otherwise diffuse reflections will scatter light out of the guiding layer. Similarly, inhomogeneities in the fiber will increase scattering of light, resulting in losses.

All ray angles for propagating waves lie between $\theta_{c}$ and $90^{\circ}$, and the corresponding effective refractive indices are in the range

$$
n_{2} \leq n_{e f f} \leq n_{1}
$$


where

$$
n_{e f f}=n_{1} \sin \theta
$$

where $\theta$ is the angle depicted in Fig. 3.4 [17]. Even thought the above mentioned conditions are met, it is not necessary that all the waves satisfying the above criteria will propagate. The core of the fiber-optic cable can be considered to be a resonant cavity. Waves propagating through such a resonant cavity will result in constructive and destructive interference patterns. The resonance conditions must be satisfied in order to maintain constructive interference and allow the waves to propagate. Hence, only certain rays are allowed to propagate and this corresponds to the modes of the waveguide. The waveguide can be single mode or multimode. Multimode transmission suffers from the problem of intermodal dispersion. There are many rays propagating in multimode fibers and each ray travels at a different speed relative to that of the other rays. The speed is primarily dependent on the angle at which the ray propagates. This results in lengthening of the optical pulses, i.e the pulses spread out in time as it travels along the length of the fiber. Apart from the number of modes that are allowed to propagate, the waves could either be transverse electric (TE) polarized, which means that the electric field is transverse to the direction of propagation of the wave, or transverse magnetic (TM) polarized, which means that the magnetic field is transverse to the direction of propagation [18].

As stated earlier, the fiber cable consists of an inner core and an outer cladding. When the refractive index of the core is uniform throughout the core and there is a sudden decrease at the junction to the cladding, it is called a step-index fiber. When the refractive index of the core gradually decreases until it reaches the junction where the core meets the cladding, it is known as graded-index fiber. Graded-index fibers reduce the problem of intermodal dispersion and have much larger bandwidths as compared with step-index fibers. Signal transmission in a fiber takes place by wavelength division multiplexing (WDM). Around 40 wavelengths, each separated by about 1 nanometer, are used as individual channels and bunched together in a single fiber in order to carry information bits. When more than 40 wavelengths are used in a single fiber, the technique is called dense wavelength division multiplexing (DWDM) and when there are less than 10 wavelengths in a channel, it is called coarse wavelength division multiplexing (CWDM). The concept of WDM is similar to orthogonal frequency division multiplexing (OFDM) [19]. 


\subsubsection{Signal Degradation in Fiber-Optic Cables}

Light rays cannot travel far in a fiber-optic cable. Repeaters have to be inserted periodically to maintain the average signal power. Signal degradation in fiber-optic cables can take place due to the following reasons [20]:

- Fiber Materials: The difference in refractive index between the core and the cladding is in the order of 0.01. To produce similar materials with such a difference in their refractive indices, trace amounts of doping material like fluorine or various oxides are added to the silica. The dopants in the fiber can lead to scattering of light and thus the loss of signal power.

- Attenuation: The attenuation of the light signal takes place due to absorption, scattering and radiative losses of the optical energy. The attenuation coefficient is generally expressed, in db per kilometer, as

$$
\alpha=10 \log \frac{P_{\text {in }} / P_{\text {out }}}{L}
$$

where $P_{\text {in }}$ is the input optical power, $P_{\text {out }}$ is the output optical power and $L$ is the length of the fiber.

Absorption is due to the fiber material and could be caused by atomic defects in the glass composition, extrinsic absorption by impurity atoms in the glass, and intrinsic absorption by the basic constituents of the fiber material. Scattering takes place due to the fiber material and also due to structural imperfections in the fiber material. Radiative losses are due to microscopic or macroscopic disturbances in the fiber geometry.

The advantages of using fiber-optics include its immunity to RF and atmospheric interference, and the massive bandwidth that it provides. A small part of the bandwidth can be utilized for usage in WAMS. The remaining bandwidth can be used by the utilities for providing secondary services like Internet access and videoconferencing, which would provide for additional revenue generation [6]. The disadvantage of using fiber-optics is its 
high initial investment and the lack of long haul digital fiber-optic systems expertise within many utilities [21].

\subsubsection{Case Studies}

Boston Edison and RCN Inc. are investing about 300 million USD in enhancing their fiber-optic network so as to provide broadband access and energy management services to the customers of Boston Edison. Utilities like Southern California Edison and Pacific Gas and Electric are also jumping into the race to provide energy management services to its customers through fiber-optic cables. Public Service Electric and Gas has teamed up with Lucent Technologies for a hybrid fiber-coax infrastructure, which would employ a variety of customer interfaces. The interfaces for such a project are provided by Honeywell [2].

\subsection{Satellites}

A satellite is primarily a repeater (dumb or intelligent) in space. It communicates with terrestrial communication centers called earth stations. Fig. 3.5 represents the concept of satellite communication with an earth station. Satellites can be classified as geostationary, medium earth orbiting, or low earth orbiting satellites. Geostationary satellites (GEO) are at an altitude of 35,786 kilometers above the equator. Satellites at this altitude rotate around the earth at the same speed of rotation as the earth and hence appear to be stationary from the surface of the earth. Medium earth orbiting satellites (MEO) are at altitudes between roughly 10,000 and 20,000 kilometers. Low earth orbiting satellites (LEO) have orbital altitudes between 750 and 1500 kilometers [22]. LEOs can provide communication links for remote areas and system installations. Some examples of LEOs include Iridium, Globalstar, and Orbcomm. Globalstar provides mobile phone services for both cellular and satellite calls, short messaging services, global roaming, fax transmissions, and data transmissions (9600bps). Orbcomm provides for tracking of remote \& mobile assets, monitoring of remote utility meters, gas storage tanks, wells \& pipelines, and communication with remote sites and personnel. 


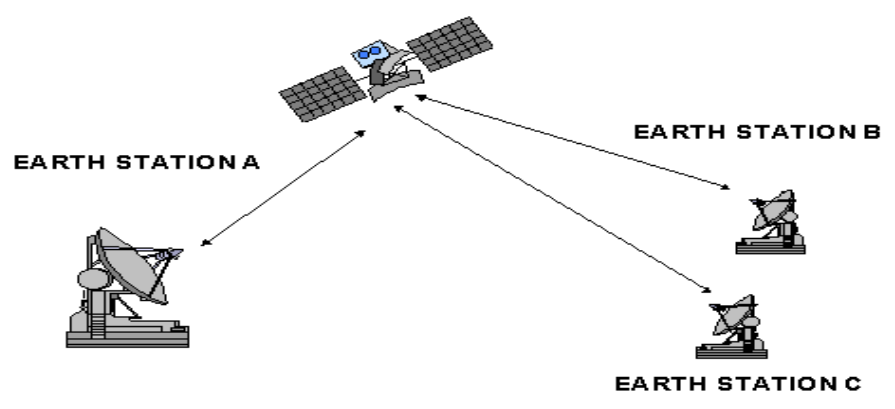

Figure 3.5: Basic satellite communication

\subsubsection{Design Considerations for Satellites}

Designing a satellite communication system is not an easy job. The type of satellite designed is a direct function of size, weight and power (SWAP). The systems that are designed today are generally power constrained and not capacity constrained. Hence an optimum satellite design is the one which minimizes SWAP and maximizes capacity and throughput.

There are various factors that have to be considered while designing a satellite communication system. These include the fact that non-geostationary satellite nodes move with time above a rotating earth, the fact that satellite network topologies change over time as the satellites cross the poles, the fact that maximum delay between two terrestrial end-points, including satellite hops, should not exceed $400 \mathrm{~ms}$ for real-time voice to avoid echo delays, the fact that routing to a called party should terminate as close to the party as possible in order to avoid costly terrestrial subscriber charges, and the fact that call setup time should not exceed 6 seconds [23]. Satellite design aspects should also include considerations like on-board battery life and on-board buffer size.

The operation of such satellites at high frequencies also generates problems of its own. As frequency increases, the effect of rain / water molecules also increases. Rain degrades signals in two ways - by attenuating the signal and by increasing the system noise 
temperature of the earth terminal. Attenuation of the signal takes place due to scattering and absorption of the electromagnetic waves. As the frequency increases, the wavelength decreases and its size becomes comparable to that of a rain drop, leading to increased chances for scattering and absorption. The system noise temperature at the earth terminal increases due to the fact that the antenna sees the warm rain / water molecules instead of the cold sky [24].

\subsubsection{Satellite Communication Architecture}

Globalstar has 48 satellites orbiting the earth at low altitudes (LEO) of about 876 miles above the earth. These satellites are placed in eight orbital planes, with each plane consisting of six satellites. These satellites are inclined at an angle of 52 degrees to provide earth coverage from 70 degrees North latitude to 70 degrees South latitude. There are eight satellites in space to provide back-up in case of emergencies and an additional twelve on earth as standby in case of launch failures. Orbcomm, on the other hand, has 35 satellites in space and these rotate above the earth at an altitude of about 600 miles. Each satellite consists of an antenna, a trapezoidal body that houses the electronics and sensors, two solar panels on each side, and a magnetometer [24]. The LEOs are generally powered by the two or more deployable solar panels, each generating about 1.1 kilowatts of power. The magnetometer on the satellite is used for attitude control and is backed up by sun and earth sensors. Fig. 3.6 indicates a schematic indicating how satellites are used by utilities for communication purposes.

Satellites communicate with earth stations called gateways. A gateway consists of three or four dish antennas having cassegrain feed and is operated by the regional service providers. A gateway can service more than one country at times. The gateways consist of dish antennas, a switching station and remote operating controls. A gateway is the most important link in the entire network as it is primarily responsible for the planning and coordination of satellite usage (Ground Operations Control Centers) and is also responsible for controlling the orbit of the satellites and their subsequent tracking (Satellite Operations Control Center). These centers also oversee satellite launches, satellite deployments, and spacecraft telemetry data. Globalstar currently uses a total of 78 gateways at present with a planned increase in the number of gateways commissioned. 


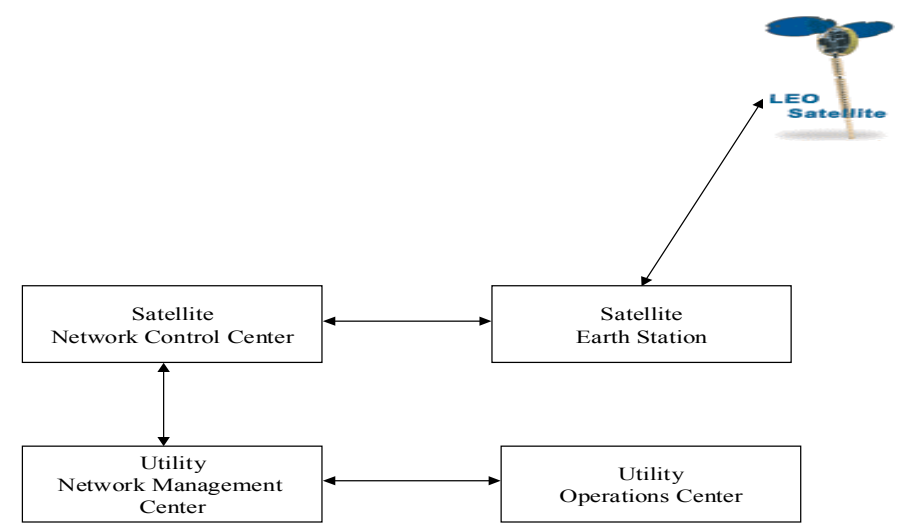

Figure 3.6: Satellite communication routing

The satellite data network center acts as a link between the network management center of the client and the earth stations of the satellite service provider. It oversees the entire operations and co-ordination between the various centers in order to ensure proper functioning of the network. The gateways offer perfect integration with local and regional telephony and wireless networks. They utilize the standard T1 / E1 interface to the existing PSTN / PLMN systems. These gateways are also responsible for the encryption of data to provide security for communications. Apart from data encryption, the entire software for the network is located at these gateways, which enable easy trouble-shooting. Satellites use techniques like bent-pipe architecture and path diversity to increase system reliability and reduce the effects of shadowing and interference [25].

The bent-pipe architecture is something akin to that of using mirrors in the sky. When a signal is transmitted, a satellite picks it up and relays the same signal, with or without signal processing, to the receiving station. In the case of buildings and terrains, which might block the signal transmission or reception, multiple satellites can be used to pick up the signal from the transmitting station and beam the signal down to the receiving station. The use of multiple satellites enables soft-handoff thereby making sure that the signal is not dropped. Using multiple satellites increases the overall reliability of the communication system. 
The main disadvantage of moving LEO satellites is that they have large Doppler spreads (on the order of tens of $\mathrm{KHz}$ in Globalstar). This large Doppler spread leads to a certain amount of uncertainty during channel search. Another characteristic of LEO satellite channels is the presence of specular reflections. Hand held user terminals may pick up strong specular reflections from surrounding large objects and this leads to a slow and cyclic variation of signal strength as a function of satellite elevation angle over time. Another disadvantage of low-earth orbiting satellites is that they have to be replaced within a span of five to eight years because of satellite damage due to radiation from the inner Van Allen electromagnetic belts.

\subsubsection{Case Studies}

Scientific Atlanta provides a fixed-site ORBCOMM satellite communicator, which supports a variety of utility automation applications like service connection, power outage detection, equipment monitoring, and security monitoring. Salt River Project (SRP) uses Orbcomm satellites to monitor and analyze vital operations data from remote locations. SRP teamed up with Orbcomm to produce SPATIA, which is interconnected to Orbcomm satellites and provides a single source system for data collection, communication and information processing ${ }^{1}$. The system provides users with near real-time data that can be used to track and collect a wide variety of inputs, including water use, water quality, energy use, and natural gas use. Satellites are also used by utilities to monitor and control remote data acquisition and status of equipment. Dancer Communications Inc. uses satellites to enable its utility customers to simultaneously monitor different operating parameters associated with pumps, generators, compressors, microturbines, and other remote equipment. Carolina Power and Light uses VSATs because these are easier to install and can withstand extreme weather conditions. Southern California Edison (SCE) developed and employed the worlds first smallest VSAT for substation SCADA [26].

\subsection{Telephone lines}

Telephone lines still form the backbone for utility communications. They offer data rates of up to $56 \mathrm{kbps}$. In substations, due to the need for isolation circuits, this

\footnotetext{
${ }^{1}$ www.orbcomm.com
} 
speed drops to a maximum of $9.6 \mathrm{kbps}$. The main advantage of using phone lines is that they are easy to set up and economical to use. Dial-up or leased lines could be considered, though the latter would be a slightly more expensive choice. Data modems are used to interface computers, computer networks, and other digital terminal equipment (DTE) to analog communication lines and radio channels. A modem is also sometimes called data communication equipment (DCE).

Modems can be classified as synchronous or asynchronous modems. Synchronous modems are faster (provide upto $56 \mathrm{kbps)}$ ) and use trellis-coded phase shift keying (PSK) and quadrature amplitude modulation (QAM). Whenever a modem is not communicating with another modem, it is said to be in command mode. The modem constantly polls the DTE for data and responds to AT command sets, which order the modem to perform a particular function. The AT command set is composed of ASCII characters and was developed by Hayes for its modems before being widely accepted.

Digital telephone circuits like T1 offer higher data rates. Digital telephone lines use digital pulses for encoding the data instead of analog signals. A T1 carrier time-division multiplexes pulse coded modulation (PCM) encoded samples from 24 voice-band channels for transmission over a single metallic wire pair or optical fiber transmission system. Each voice-band channel has a bandwidth of approximately $300 \mathrm{~Hz}$ to $3000 \mathrm{~Hz}$ [27].

\subsection{Microwave Links}

Microwaves are generally described as electromagnetic waves with frequencies that range from approximately $500 \mathrm{MHz}$ to $300 \mathrm{GHz}$ or more. Terrestrial microwave systems use frequency modulation (FM) or digitally modulated (PSK or QAM) signalling.

Point to point microwave links are being used by utilities to a great extent. Microwave links provide a better option as compared to leased lines, since they are easy to set up and are highly reliable. Digital microwave links are set to replace analog links in the near future. Digital microwave links fall into two categories: frequency bands less than $10 \mathrm{GHz}$ and frequency bands greater than $10 \mathrm{GHz}$. Bands which are below $10 \mathrm{GHz}$ have long propagation distances up to 30 miles. Bands over $10 \mathrm{GHz}$ are distance limited to 
less than 5 miles. The main disadvantages of using microwave links are signal fading and multipath propagation. To serve the needs of the utilities, high speed data rate capability and noise immunity of digital microwave links can be preferred over analog microwave links [21]. Analog microwave links are fast becoming obsolete in todays digital era. Repeaters are used for long haul communication purposes and diversity techniques are employed to improve the signal to noise ratio (SNR). Kansas City Power \& Light has opted for a wireless communications system to provide energy management services to its utilities. Allegheny Energy uses microwave links to communicate between substations and control stations.

\subsection{Power Lines as a Communication Medium}

Power lines exist along the length and breadth of any country and can provide a reliable and fast means for communications due to their ubiquitous nature. They also offer last-mile conductivity and data rates on the order of Mbps and hence can provide a cost-effective solution. But power lines were never really made for transmission and reception of data and hence provide a hostile environment to their use as a medium / channel. The disadvantages associated with transmission over power lines are high noise levels, high signal attenuation and distortion, and the inability of high frequency signals to pass through distribution transformers.

Power line communications has immense potential in broadband communication applications and can be considered to be an economical option as compared to fiber optics and satellite transmissions that are currently being used for broadband communications. Fig. 3.7 indicates the basic concept of power line communications (PLC). The power signal is the $120 \mathrm{~V} \mathrm{p}-\mathrm{p} 60 \mathrm{~Hz}$ signal. The data signal can be transmitted at frequencies ranging from $20 \mathrm{kHz}$ to $4 \mathrm{MHz}$. Filters are used to isolate the power and data signals. High pass filters are used to decouple the data signal from the power signal and the data signal is then forwarded for additional processing.

Power lines will definitely offer an economic communication link for broadband communications in the future. Fairly good data rates provide for an excellent communication link by way of power lines. Power line communication (PLC) can be considered for use in wide area measurement systems (WAMS) because they offer data rates on the 


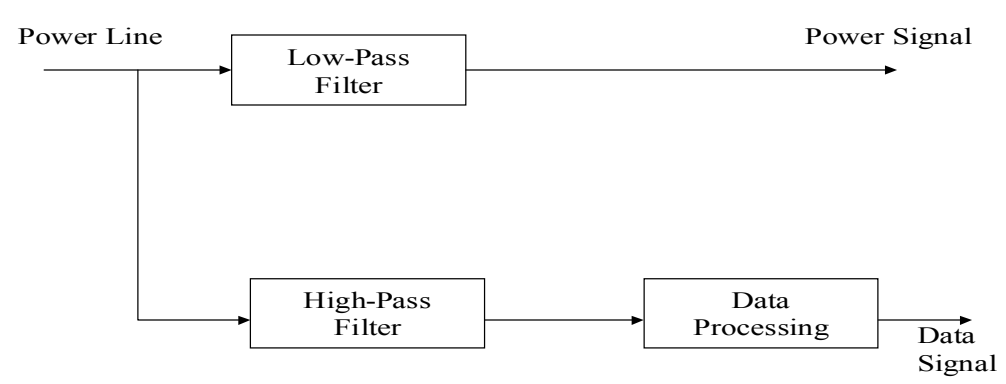

Figure 3.7: Basic concept of power line communications

order of $4 \mathrm{Mbps}$ via the electricity supply grid. Power line technology uses the medium and low voltage electric supply grid for transmission of data and voice. It can be used for both WAMS communication requirements and substation networking [28]. Using spread spectrum techniques like direct sequence spread spectrum (DSSS) and frequency hopping spread spectrum (FHSS) or orthogonal frequency division multiplexing (OFDM) can result in tolerance of self interference due to multipath propagation and also interference due to co-channel or adjacent channel signals, thereby improving bit error rate (BER) [29].

Nortel Communications is one of the leading players in the area of power line communications. Nortel has completed many trial PLC installations in England, enabling broadband access to residential homes. Asea Brown Boveri (ABB) has also introduced telecommunication systems that can be used for real time communications over power lines. Intellon Corporation is best known for its research in the area of power line communications. Intellon is the manufacturer of many integrated circuits (ICs) that are presently being used by companies which are working towards developing PLC broadband solutions. Telecommunication giant Motorola is also researching its entry in this sector of telecommunications. Detroit Edison has invested around 10 million USD in Echelon Corporation, the company that is one of the front runners in power line communications. Major utilities like Southern Company, Pacific Power \& Light (PPL), and Potomac Electric \& Power Company (PEPCO) are also involved in power line communications. 


\subsection{Conclusion}

The chapter presented a survey of different types of communication links that are used by utilities for their communication needs. The chapter provided an overview of each type of communication link with special focus on fiber-optic cables and satellites as these are the major types of communication links employed by utilities today. PLC, an emerging communication technology, was also highlighted. Case studies were presented, illustrating how power companies are currently using advanced communication technologies and are moving away from legacy communication systems. 


\section{Chapter 4}

\section{Communication Delay in WAMS}

\subsection{Introduction}

Wide area measurement systems (WAMS) will dynamically alter the monitoring and control scenario of the power network grid in the near future. WAMS control, in almost real-time, the oscillations and transient instabilities in the grid. The bottom line however is that an effective and reliable communication link is required to ensure a minimal time lag between monitoring and controlling the situation. The effectiveness of WAMS is greatly dependent on future technologies like flexible AC transmission systems (FACTS) and communication delays in the information network.

The type of communication link involved and the length and processing of messages in the information network determines the overall latency of the system and its ability to react almost instantaneously to outages and transients in the power grid. If not effectively controlled, local outages could lead to cascaded outages, which could result in large area blackouts. Such blackouts could prove detrimental to the market competitiveness of the utility in general and the economy as a whole. It is therefore necessary to ensure the effective utilization of communication links, future technologies, and advanced concepts like WAMS. 


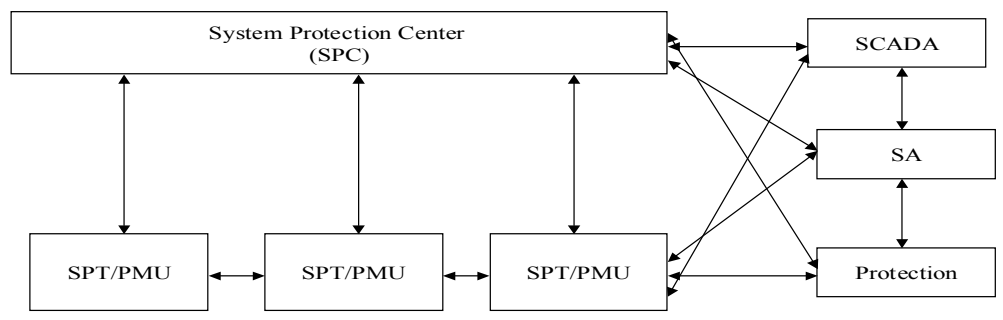

Figure 4.1: General system setup of WAMS

\subsection{Communication Issues in WAMS}

WAMS help to monitor and control cascaded outages. WAMS offers almost realtime protection against transients and oscillations that could cause the grid to be unstable. Fig. 4.1 offers a system setup view of WAMS [7].

The system protection center (SPC) offers a dynamic view of the state of the system and is responsible for the monitoring and control of large power networks. The system protection terminals (SPTs) are smart terminals and are local control points in the larger picture provided by SPC. The phasor measurement units (PMUs) are measuring devices that are present at the SPTs. Supervisory control and data acquisition systems (SCADA) can work closely with PMUs to ensure proper monitoring of the grid. The substation automation (SA) network is responsible for maintaining effective information transfer between the various participants in WAMS. Monitoring and Control algorithms are run in the SPC, though parts of these algorithms can also be run at local SPTs in a distributed manner. Distributed management of algorithms results in minimal time lag before action is initiated to bring grid instabilities under control.

Coordination between different SPTs is crucial to maintaining grid stability. Small signal angle instabilities can be controlled more effectively by SPTs running local algorithms, but voltage and frequency instability require a wide system view. Correction techniques, for 
voltage and frequency instabilities include load shedding, generation acceptance/rejection, and coordinated islanding of the grid. To ensure that correction actions are effective, a wide area system view of the states is required, which would offer information regarding which power units are operating at which level and what is the actual load pattern and level. Remedial actions for voltage and frequency instabilities can only be optimized if the actual situation of the observed area is known [7].

WAMS enhance system reliability by early detection and immediate avoidance of possible catastrophic events. The economic advantages of WAMS include the fact that it is an appropriate system for business support in an unbundled and open access utility environment, that it is designed for an open-access market where production and transmission patterns will more often vary than in a closed market, and that it creates maximum profitability by reducing downtime and by optimizing system performance.

PMUs play a crucial role in measuring the actual state of the system. PMU based state estimation is a linear model and hence is much faster than conventional nonlinear state estimation models. The information about a certain area can be easily determined by a PMU based state estimation [30].

The technology of phasor measurements plays an important role in determining the effectiveness of WAMS. Apart from technology, the other aspect that plays an important role in determining the effectiveness of WAMS is the communication delay that various types of communication links impose on the system. Systems with large communication delays exhibit large time delays before correcting instabilities. Such time delays could cause corrective measures to fail and local outages could end up becoming cascaded outages. As stated earlier, communication delays are based on the type of communication link involved, the length of the message, in this case the information transfer between the PMU and control stations, and the processing involved at respective stages of information transfer. PMUs communicate with control stations based on the IEEE 1344 Synchrophasor standard, which was described in detail in Chapter 2. In brief, the PMU transmits messages (according to the IEEE 1344 format) in three types of frames: 
- Data frame: The data frame provides information regarding phasor data and the state of the digital inputs on each channel. It also defines the trigger status of frequency, angle, over-current, under-voltage and rate of frequency change.

- Header frame: The header frame is an ASCII file that contains identification information about the PMU, data source, algorithms, and the analog filters used.

- Configuration frame: This is a machine-readable binary file that contains PMU identification code, number of phasors, number of digital channels, channel and phasor names, nominal line frequency, and the transmission period of the phasors.

The PMU also receives messages that indicate when to start or stop the transmission of data from the PMU. The data format for this primarily consists of the time-tag and the command code, which informs the PMU of the action that needs to be taken [10]. Time-tagging is very essential for PMUs since it helps in providing almost real time data to the control centers and helps identify the instantaneous state of the system something that conventional SCADA systems cannot do. Many utilities are seriously considering the implementation of PMUs in WAMS. Southern California Edison (SCE) has implemented a trial run of up to 32 PMUs in the state of California. Fig. 4.2 represents the SCE case study and provides a system overview of PMU implementation.

\subsection{Communication Delays}

Communication links used by WAMS include both wired (telephone lines, fiberoptics, power lines) and wireless (satellites) options. Delays associated with the link act as a crucial indicator to the amount of time lag that takes place before action is initiated. The delays are an important aspect and should be incorporated into any power system design or analysis, as excess delays could ruin any control procedures adopted to stabilize the power grid. The communication options for WAMS include fiber- optic cables, satellites, analog and digital telephone lines, point-to-point microwave links, and power lines. 


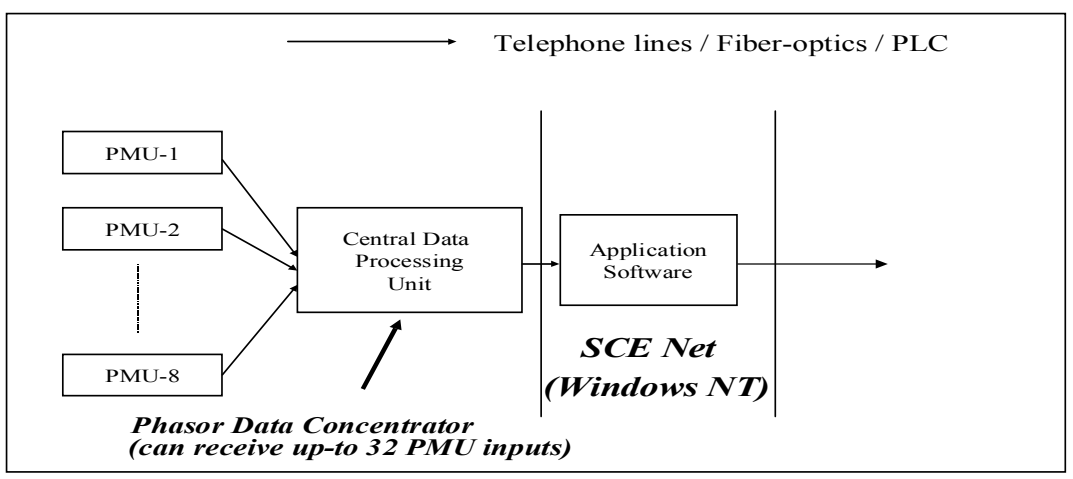

Figure 4.2: System overview of PMU implementation by SCE

\subsubsection{Communication Delay Causes}

Unavoidable time delays in distributed control systems impact the performance of the system as a whole in its ability to efficiently control system instabilities [31]. Communication delays primarily depend on the type of communication link involved, the length of messages used in the information transfer, for the purpose of monitoring and control, and the information processing involved. Fig. 3 indicates the delays in a power system. In the case of PMUs using the IEEE 1344 data format, the communication delay causes can be classified as [32]:

- Phasor transducer (PT) and voltage transducer (VT) delays: A transducer is a processing device that instantaneously converts points on analog signal waveform into measurable terms like root mean square (RMS) voltage, RMS current, RMS power, waveform frequency, and relative angles for current and voltage. In other words, Transducers are used to measure the RMS voltages and currents at the instant of sampling. Digital transducers are currently being used because these are more immune to noise and extraneous factors when it comes to measuring the voltage or current waveforms RMS values.

- Window size of the DFT: Window size of the discrete Fourier transform (DFT) is the number of samples required to compute the phasors using DFT. Window size 


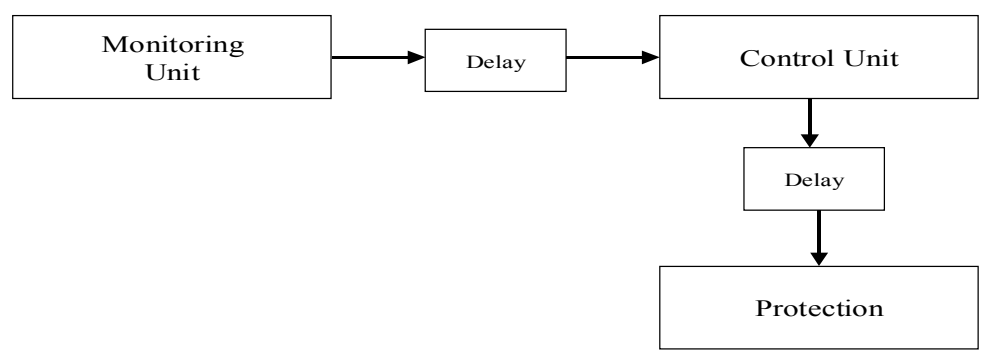

Figure 4.3: Delays in a power system

plays an important role in determining the amount of delay. It is obvious that smaller window sizes will produce lesser delay, but the problem here is that smaller window sizes leads to larger area of uncertainty in calculation of the phasor values. Larger window sizes will no doubt increase the amount of delay, but will have minimal uncertainty regarding phasor values. A balance has to be maintained between window size and reliability of phasor information.

- Processing time: The processing time required in converting the transducer data into phasor information with the help of DFT. Processing mainly involves the use of DFT in calculating the fundamental frequency component of the sample voltage or current. Filters that are used before and after processing, also contribute to the delay.

- Data size of the PMU output: Data size of the PMU message is the size of the information bits contained in the data frame, header frame and the configuration frame. The length of the message influences the amount of delay because longer the message size, longer will be the delay and vice-versa. The data is assumed to travel close to the speed of light in a fiber-optic cable and two-thirds the speed of light in a copper wire. 
- Data error-coding: The data or message that is transmitted is encoded by cyclic redundancy check (CRC) to ensure that the message is received correctly at the receiving station. Other forms of coding like Reed-Solomon codes are also used, by satellites, to reduce the bit error rate (BER) of the communication system. The encoding and subsequent decoding of data also adds to the amount of delay.

\section{- Transitions between the communication link and the data processing equip-} ment: Transitions between the communication link and the data processing equipment leads to delays that are caused at the instances when data is retrieved or emitted by the communication link. Transitions between packet switched networks and circuit switched networks also adds to the transition delay. Hybrid technologies could also produce bottlenecks when digital networks like fiber-optic cables are used alongside analog telephone lines.

- Communication link involved: The communication link involved could be anything from a fiber-optic cable to a power line cable. The type of communication link and the physical distance involved in transmitting the PMU output to the central processing unit can add to the delay. For long-haul communications, repeaters are required to boost the average signal power because of signal losses and attenuation during transmission through the medium. The use of repeaters, with increase in the physical distance, adds to the delay.

- Data concentrators: Data concentrators are primarily data collecting centers located at the central processing unit and are responsible for collecting all the PMU data that is transmitted over the communication link. Data concentrators collect the data and forward the same to monitoring and control algorithms that run at the system protection center, for analysis and decision-making purposes.

\subsubsection{Communication Delay Calculations}

Delay calculations form an important aspect of WAMS. These delays indicate the viability of a particular communication medium, since large communication delays amount 
to slower controller actions that can correct power grid instabilities and oscillations. Communication delay can be expressed as

$$
\tau=\tau_{f}+\tau_{p}+\frac{L}{R}+\theta
$$

where $\tau$ is the total link delay, $\tau_{f}$ is the fixed delay associated with transducers used, DFT processing, data concentration and multiplexing, $\tau_{p}$ is the link propagation delay, $L$ is the amount of data transmitted, $R$ is the data rate of the link, and $\theta$ is the associated random delay jitter.

Delay calculations can be made on the assumptions that there are 1012 phasor measurements, each 4 bytes in length, 10 input status channels, each 2 bytes in length, and the combined delay caused by processing, concentrators, multiplexing and transducers is estimated to be around $75 \mathrm{~ms}$. This is the fixed delay and is independent of the communication medium used.

The propagation delay is dependent on the medium and thus is a function of both the medium and the physical distance separating the individual components of WAMS. We have assumed that media like fiber-optic cables, power lines, and telephone lines, on an average, have a propagation delay of around $25 \mathrm{~ms}$. In the case of LEO satellites, the propagation delay could be as high as $200 \mathrm{~ms}$, because of the distance involved and gateways that connect terrestrial networks to the satellite. There would be further delay involved if there is some processing and queuing at the gateways or if there is some on-board processing performed by the satellite itself. There is also additional delay caused by the amount of data that has to be transported across the medium and the data rate of the medium. There could be additional delay in satellite transmission because LEOs generally use multiple satellites for communication and, inter-satellite communication and routing through the network, could add to the delay.

The analog to digital converter (ADC) in the PMU has 16-bit resolution and sampling is assumed to be 720 samples per second. For the PMU, it is estimated that the length of the data frame is around 640 bits, the length of the header frame is around 200 bits and the length of the configuration frame is around 2800 bits. The normal operation of the PMU requires only the transmission of the data file. The header and configuration files can 
be transmitted at equipment startup or on a request basis. For worst-case delay scenarios, we have assumed that there is a regular transmission of the header and configuration files along with the data files.

Considering the total length of the PMU packet, the delay caused due to the amount of data transmitted and the data rate, can then be estimated to be around $125 \mathrm{~ms}$, considering a telephone line channel with an average capacity of $33.6 \mathrm{kbps}$. In the case of substations, where isolation circuits are required, the telephone line channel capacity could drop to as low as $9.6 \mathrm{kbps}$, creating a bottleneck, and thereby increasing the delay to more than $200 \mathrm{~ms}$. Even though power lines can reach speeds of up to $4 \mathrm{Mbps}$, delay calculations due to the amount of data transmitted and the data rate, can be assumed to be similar to telephone line delay calculations. This assumption is valid because power lines suffer from an enormous amount of interference and satisfactory BER performance would require data rates in the range of a few kbps.

Fiber-optic cables do not contribute to the delay associated with the length of the PMU packet because of their high data rates, and hence the associated delay can be considered to be negligible. The responsivity and sensitivity of photodetectors used in fiber-optic transmission can cause some amount of delay, but for all practical purposes can be considered to be negligible. The delay analysis of digital microwave links would be similar to that of fiber-optic channels because the data rate for the former can be considered to be unlimited for all practical purposes [21]. The length of the PMU packet also introduces additional delay in the case of satellites because of narrow bandwidth channels, noise, and interference.

Table 4.1 shows the delays of various communication links when using PMUs in a WAMS environment and could provide useful delay statistics that can be integrated into simulation and performance analysis of WAMS. All the above-mentioned communication link options can provide for almost real-time correction of unstable situations that could lead to large area cascaded outages. The advantages and disadvantages of each communication link have to be weighed by the utilities and then the right link has to be chosen based on the required control dynamics and operating economics of the power system. 
Table 4.1: Delay calculations associated with various communication links

\begin{tabular}{|l||c|}
\hline Communication link & $\begin{array}{c}\text { Associated delay- } \\
\text { one way (milliseconds) }\end{array}$ \\
\hline Fiber-optic cables & $\approx 100-150$ \\
\hline Digital microwave links & $\approx 100-150$ \\
\hline Power line (PLC) & $\approx 150-350$ \\
\hline Telephone lines & $\approx 200-300$ \\
\hline Satellite link & $\approx 500-700$ \\
\hline
\end{tabular}

\subsection{Conclusion}

The communication delay causes were studied in this chapter. Communication delay measurements due to the usage of PMUs in measurement systems are provided for various communication mediums. These results could be integrated into realistic power system modeling, design and analysis, which would determine the effectiveness of WAMS in the real-time control of the power grid. 


\section{Chapter 5}

\section{Power Line Communications - Overview and Channel Model}

\section{$5.1 \quad$ Introduction}

Power lines are offering new communication options for the future. Utilities can take advantage of power line communications (PLC) for communication purposes. Power line communication equipment can be used as part of a dynamic wide area protection system (WAP). PLC can be used as a viable and economic option for communicating between SCADA / RTU terminals and area control centers. PLC has been used especially in developing countries like Guam. Guam Power Authority (GPA) uses PLC as a backup to its fiber-optic communication network.

Power line communications has immense potential for broadband communication applications and can be considered to be an economical option as compared to fiber optics and satellite transmissions that are currently being used for broadband communications. This chapter provides an insight into power lines as a communication medium by inspecting the characteristics of power lines as a carrier and concludes by suggesting a channel model.

\subsection{The Electric Power Supply}

The electric power supply is classified based on the voltage levels of the transmission cables. Transformers that ensure correct voltage levels and minimum energy losses 
interconnect the transmission cables.

\subsubsection{Transmission Line Classification}

The transmission lines can be classified according to their voltage magnitudes as follows [33]:

- High-Voltage Cables: The high voltage cable carries voltage levels in the range $110-380 \mathrm{kV}$ and is primarily used in transporting electric power over large distances, which could be as large as several hundred kilometers. High voltage lines are intended to reduce transmission loss over long distances and the core is generally constructed using aluminum. The transmission losses include heat loss due to the ohmic resistance of the cable material and leakage loss due to leakage currents in the insulator, which covers the core of the cable. The leakage losses are much smaller than the heat losses and are affected by climatic conditions and the earths thermal resistivity in the case of buried power cables.

At high voltage levels, apart from heat loss and leakage loss, corona loss also plays an important part in transmission losses. Corona loss is due to the discharge of air particles around the cable and occurs because of the intense electric field around the high voltage transmission cable. Coronal discharge occurs if the surface voltage gradient of the transmission cable is greater than the earths dielectric rating which is around $30 \mathrm{kV} / \mathrm{cm}$. Coronal loss not only contributes to power loss, but also interferes with radio reception.

- Medium Voltage Cables: The voltage levels in medium voltage cables vary between 10-20 kV. These generally supply electric energy to rural areas, small towns, industries, and plants. The length of typical medium voltage cables varies between 5 and $25 \mathrm{kms}$.

- Low Voltage Cables: The voltage level in the low voltage cables is around 120/240 $\mathrm{V}$ and these are used for connecting the power grid network (via high level and medium 


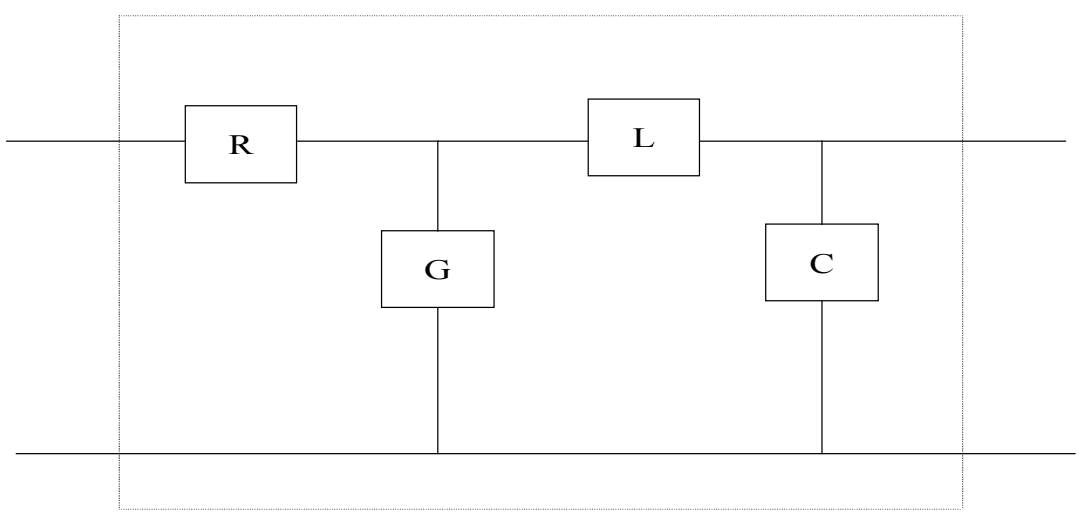

Figure 5.1: Transmission line characteristics

level voltage cables) to residential buildings. The supply radius of low voltage cables is between 100 and 500 meters. The core is constructed using materials like copper and aluminum. The insulator is usually either polyvinyl chloride (PVC) or vulcanized polyethylene (VPE).

\subsubsection{Transmission Line Modelling}

Fig. 5.1 characterizes a typical transmission line. $\mathrm{R}$ is the resistance of the transmission line, $\mathrm{L}$ is the inductance, $\mathrm{G}$ is the conductance, and $\mathrm{C}$ is the capacitance.

The characteristic impedance of the transmission line is given as:

$$
Z_{L}=\sqrt{\frac{R+j \omega L}{G+j \omega C}}
$$

The characteristic impedance presents the impedance of the transmission line to the power generation and distribution system. It provides power system designers with an estimate of system impedance levels. Power system impedance levels are important, because for efficient power distribution, the impedance of the source (generation and transmission) must be very small when observed from the load side.

The other important factor that needs to be considered while designing the transmission system is the propagation constant given by 
Table 5.1: Transmission line parameters - approximate

\begin{tabular}{|l||c||c||c|}
\hline Type of Cable & Inductance-mH/km & Capacitance-nF $/ \mathbf{k m}$ & $Z_{L^{-}}$ohms \\
\hline High-voltage & 0.813 & 13.890 & 242.77 \\
\hline Medium-voltage & 0.400 & 00.500 & 33.00 \\
\hline Low-voltage & 0.310 & 00.800 & 27.00 \\
\hline Overhead-lines & 1.57 & 00.013 & 358.00 \\
\hline
\end{tabular}

$$
\gamma=\alpha+j \beta
$$

where $\alpha$ is the attenuation constant and $\beta$ is the phase constant. The attenuation constant provides an insight into the signal attenuation properties of the transmission line and the phase constant indicates the amount of phase change the signal undergoes while passing through the length of the transmission line.

The propagation constant is specified, in simpler terms, as

$$
\gamma=\sqrt{(R+j \omega L)(G+j \omega C)}
$$

The above equations can be combined in order to obtain the phase constant and the attenuation constant. The attenuation constant is approximated as

$$
\alpha \approx \frac{R}{2 Z_{L}}+\frac{G Z_{L}}{2}
$$

and the phase constant is approximated by

$$
\beta \approx \omega \sqrt{L C}
$$

Table 5.1 indicates certain approximate values of typical transmission line parameters for different types of transmission lines [33]. 


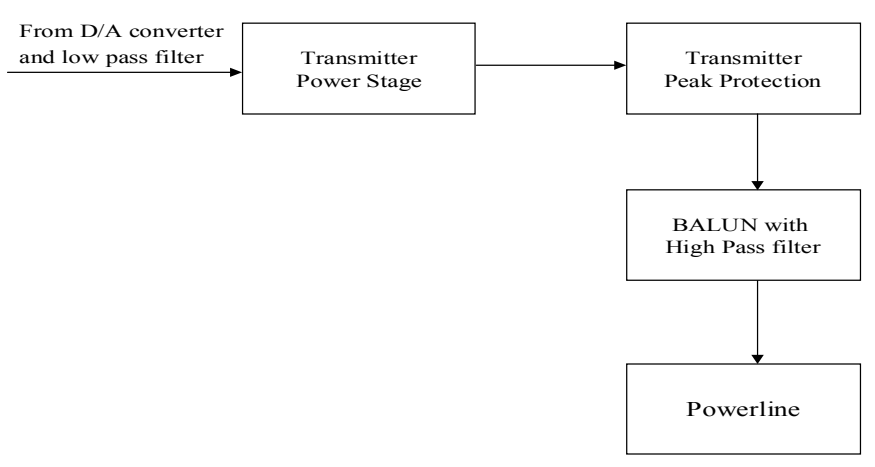

Figure 5.2: Transmitter side coupling

\subsection{Concept of Power Line Communications}

The power signal is basically a $60 \mathrm{~Hz}$ signal. The data signal can be transmitted at frequencies ranging from $20 \mathrm{kHz}$ to $250 \mathrm{kHz}$ and more. Companies like Intellon and Inari have developed PLC systems that operate in frequency bands, in the range of a few $\mathrm{MHz}$. Filters are used to isolate the power and data signals. High pass filters are used to decouple the data signal from the power signal and the data signal is then forwarded for additional processing. Signal coupling of the information data into the power network is an important aspect of PLC.

Signal coupling, generally implemented in parallel, is an important aspect of power line communications. The signal has to be in a frequency band that is much higher than that of the mains frequency and most of its harmonics, and at the same time, the signal must have enough power so that it can be injected into the power line medium. A transformer (BALUN balanced unbalanced) with high pass characteristics can handle coupling to the mains. The high pass filter will ensure that the main power and most of its harmonics are kept away from the modem. Fig. 5.2 indicates the signal coupling at the transmitter side.

The final stage of the transmitter receives the analog signal that has to be transmitted from the D / A converter. The coupler has to transport this signal without loss and at the same time, it has to ensure isolation with the mains voltage. 


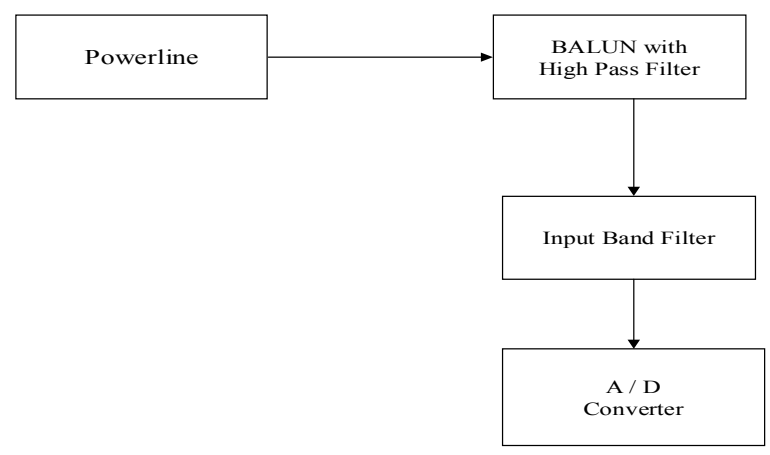

Figure 5.3: Receiver side coupling

Coupling at the transmission side is quite strong, but the coupling at the receiver side is loose. Loose coupling has the advantage of reducing interference effects on the signal. Fig. 5.3 shows the signal coupling at the receiver side. The transformer provides for a safe separation of the signal from the mains.

The methods suggested above work well for frequencies less than $150 \mathrm{kHz}$, but for frequencies above $150 \mathrm{kHz}$, additional circuitry has to be implemented in order to allow the coupling of the signal through the transformers. Coupling is also important to prevent power line modems from being damaged by fast transients in the mains. Transients have sufficient energy to damage electric circuitry and hence proper protection coupling has to be maintained. Suppressor diodes can be used to protect transmitter and receiver modems from transients in the mains. The suppressor diodes are fast acting and have sufficient capacity to convert large amount of power into heat without being damaged. The only problem with such diodes is that these are not effective at very high frequencies and hence special protection circuits have to be designed to protect against transients at high frequencies.

\subsection{Characteristics of the Power Line Channel}

Power lines exist along the length and breadth of any country and can provide a reliable and fast means for communications due to their ubiquitous nature. They also 
offer last-mile conductivity and data rates on the order of Mbps and hence can provide a cost-effective solution for communication purposes. However, power lines were never really made for transmission and reception of data and hence provide a hostile environment to their use as a medium / channel. The disadvantages associated with transmission over power lines are high noise levels, high signal attenuation and distortion and the inability of high frequency signals to pass through distribution transformers.

Power lines can be broadly classified into transmission lines and distribution lines. Transmission lines are used to transport power from the generator to the distribution substations, where they branch out into individual homes, at this point the lines are called as distribution lines. Transmission lines offer a better medium of communications as compared to distribution lines because the former does not have any time-varying loads connected to it [34]. Hence it is easier to model transmission lines as compared to distribution lines, for communication purposes. The characteristics of power lines include:

1. Attenuation : Attenuation in power line networks depends significantly on network load and is partially selective, exhibiting extreme variance with time [29]. Signal attenuation is also dependent on the frequency of the signal $f_{o}$ and the transmitterreceiver seperation $d$. Signal attenuation increases with the frequency of the signal and the distance between the transmitter and the receiver. For frequencies below 400 $\mathrm{kHz}$, the variation with frequency is rather slow and stable and the attenuation is 3 to $25 \mathrm{~dB}$. On the other hand, at frequencies above $400 \mathrm{kHz}$, the attenuation varies very sharply with frequency [35]. An approximate attenuation factor is indicated in [36] and is represented here as

$$
\alpha\left(f_{o}\right)=a_{0}+a_{1} f_{o}^{k}
$$

where $\alpha$ is the attenuation factor, $a_{0}, k$, and $a_{1}$ are measured parameters, and $f_{o}$ is the frequency of operation. The attenuation $A$ can then be approximated as [36]

$$
A=e^{-\left(a_{o}+a_{1} f_{o}^{k}\right) d}
$$

2. Varying Impedances: The transmitter modem has to inject the data signal into the mains with sufficient power, which will enable the signal to reach the receiver. 
The required transmission power can be easily calculated when the input impedance of the mains is known. The smaller the impedance, the more power is required [33]. The impedance of the power outlet is determined primarily by appliances connected to the wiring and by the impedance of the distribution transformer [37]. Impedances vary according to the appliances connected, frequency of signal and the distribution transformer impedance. Statistically speaking, the impedance of the power line varies from 2 to $30 \mathrm{ohms}$ below $1 \mathrm{MHz}$ and between 20 and $200 \mathrm{ohms}$ for frequencies greater than $1 \mathrm{MHz}$ [38]. In [35], the following estimate was given for the average input impedance of a power line

$$
|Z|=0.005 f^{0.63} \Omega
$$

where $f$ is the frequency of the signal.

3. Impulsive Noise: Impulsive noise is generated by dimmers and motors and appears as a transient high-energy noise covering a wide frequency range (up to $500 \mathrm{KHz}$ ) [39]. In general dimmers using fast switching triacs produce greater noise levels than universal motors with brushes. Impulses are generally found with a repetition rate between 50 and $200 \mathrm{kHz}[33]$.

4. Harmonics: Switching power supplies in appliances cause harmonics (above $50 \mathrm{kHz}$ ) and hence produce continuous wave $(\mathrm{CW})$ jammers.

5. External influences: Varying levels of noise and interference are generated in transmission power lines due to broadcast signals from TV and radio stations and due to corona discharge from the sun.

6. Impedance modulation: Rectifier circuits cause amplitude modulation of the signal. Rectifiers can turn on once or twice during a $60 \mathrm{~Hz}$. cycle. When these turn on, they connect a voltage capacitor directly across the power circuit. This affects the impedance as seen by the receiver or the transmitter placed on the power line causing it to change at $60 \mathrm{~Hz}$ or $120 \mathrm{~Hz}$ cycle. The signal voltage is correspondingly affected 


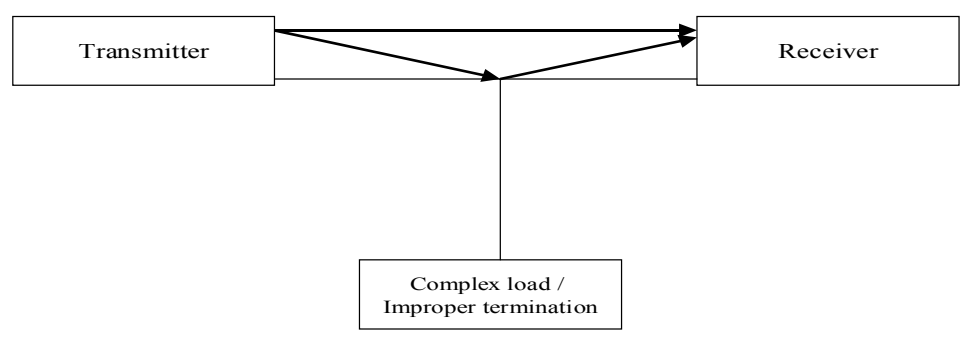

Figure 5.4: Multipath propagation

by this changing impedance and gets amplitude modulated.

7. Problem with complex loads: Complex loads form resonant circuits creating a notch filter effect at their resonant frequencies. Also improper load termination leads to reflections creating standing wave patterns (see fig. 5.4. This can lead to multipath components similar to those produced in wireless channels. The multipath impulse response can be described as

$$
h(t)=\sum_{i=1}^{N} c_{i} \delta\left(t-\tau_{i}\right)
$$

where $N$ is the number of multipath components of the signal, $c_{i}$ is the weighting factor associated with each signal component such that

$$
\left|c_{i}\right| \leq 1
$$

The reason that the weighting factor is less than one is the fact that multipath transmission occurs only at joints, where the load of a parallel connection of two or more cables leads to a resulting impedance being lower than the characteristic impedance of the feeding cable [36]. When there are more signal reflections, the value of the weighting function $c_{i}$ will be smaller. The delay $\tau_{i}$ can be calculated as

$$
\tau_{i}=\frac{d_{i} \sqrt{\epsilon_{r}}}{c_{o}}
$$


where $\epsilon_{r}$ is the dielectric constant of the insulating material, $d_{i}$ is the signal path length, and $c_{o}$ is the speed of light.

8. Time-varying channel: The noise is minimized during the zero crossing of the power signal. It is generally at the peak of a power signal when rectifiers and switching circuits switch in order to function accordingly. These switching actions have an adverse effect on data communications as these lead to transient noise in the power line. Thus, the channel is highly nonstationary [40].

9. Distribution transformer: When we consider power line communications, we also have to consider the fact that high frequency signals that are used for intra-building purposes cannot be used for transmitting beyond the distribution transformer, which supports them. This can be both advantageous as well as disadvantageous. Advantageous, because the distribution transformer would offer perfect isolation for LAN networks on the secondary side of the transformer. Disadvantageous, because bypass cables and isolation circuits will have to be used to interconnect networks which are on either side of the distribution transformer and this could affect data transfer rates.

\subsubsection{Attenuation}

Attenuation is directly proportional to the frequency of operation $f_{o}$ of the communication system. This is due to the fact that at higher frequencies, the skin depth of the conductor (power line cable) reduces, leading to an increase in resistance of the conductor material, which in turn reduces the signal power. Normally, aluminum is used as a conductor especially for high voltage lines. In the case of aluminum, the skin depth $a$ is given as

$$
a \approx \frac{8.768}{\sqrt{f_{o}}}
$$

Apart from the skin depth, atmospheric conditions can also lead to increased fluctuation of the signal. The attenuation in a power line $A$, without considering atmospheric 
effects, can be approximated as

$$
A=e^{-\alpha\left(f_{o}\right) d}
$$

where $\alpha$ is the attenuation constant of the conductor at the frequency of operation (per $\mathrm{km})$ and $d$ is the physical seperation between the transmitter and the receiver, in meters.

\subsubsection{Impulsive Noise}

Implusive noise due to switching circuits or universal motors can also cause substantial interference to the data signal. Techniques like SS and OFDM could be used to mitigate the effects of impulsive noise. Interference also exists in the form of corona discharge and broadcast signals. The impact of such interference is much more critical than that of impulsive noise interference because they are constantly present and that too at relatively high levels [41]. This type of interference can be modeled as additive white Gaussian noise. Impulsive noise in power lines can be broadly classified depending on the duration of the impulse. The classification is described by Table 5.2.

Table 5.2: Impulse noise classification

\begin{tabular}{|l||c||c||c|}
\hline Impulse Energy & High & Medium & Low \\
\hline Class & 1 & 2 & 3 \\
\hline Impulse duration & $65 \mu s$ & $20 \mu s$ & $5 \mu s$ \\
\hline Repetition rate & $120 s^{-1}$ & $200 s^{-1}$ & $400 s^{-1}$ \\
\hline
\end{tabular}

From the table it seems that impulsive noise of class 3 would do the most damage since it is highly repetitive, but due to the low pass nature of the electric supply networks, the class 3 impulse noise suffers higher attenuation. On the other hand, class 1 impulse noise have high energy and can cause the worse interference to the data signal.

\subsubsection{Impedance Mismatch}

Signal transitions between conductors having different impedances can lead to reflections that result in loss of signal power. When two lines with different characteristic impedances $Z_{L 1}$ and $Z_{L 2}$ are coupled and if each line is terminated with its characteristic 
impedance, then the resulting reflection factor $r$ at the coupling point is given by

$$
r=\frac{Z_{L 1}-Z_{L 2}}{Z_{L 1}+Z_{L 2}}
$$

For example, the characteristic impedance of a ground cable $Z_{L 1}$ is around $50 \Omega$ and the characteristic impedance of an overhead line cable $Z_{L 2}$ is around $470 \Omega$ [33], then $r$ can be calculated as

$$
\begin{aligned}
r & =\frac{50-470}{50+470} \\
& =-0.8
\end{aligned}
$$

The ratio of the tranmitted power $P_{t}$ to the injected power $P_{i}$ is given as

$$
\frac{P_{t}}{P_{i}}=1-|r|^{2}
$$

and the reflected power $P_{r}$ is given as

$$
P_{r}=|r|^{2} P_{i}
$$

This means that only about $36 \%$ of the signal power will pass through whereas about $64 \%$ os the signal power will travel back to the source.

High frequency balanced-unbalanced (BALUN) transformers with the appropriate number of turns can be used to create a signal transition free from reflections. In the example mentioned above, a transformer with a turns ratio of $3\left(\sqrt{\frac{470}{50}}\right)$ can be used to prevent signal reflections caused due to the impedance mismatch between the connecting cables.

\subsection{Power Line Channel Model}

The Power line channel model can be implemented for long distance transmission cables and for short distance residential power system wiring. It is easier to implement a model for long distance transmission lines as the power line channel can be modeled as an additive white Gaussian noise (AWGN) channel since transmission lines offer a less noisy channel as compared to residential power lines. Apart from the AWGN characteristics, signal attenuation would probably be the only main factor during modeling a transmission 


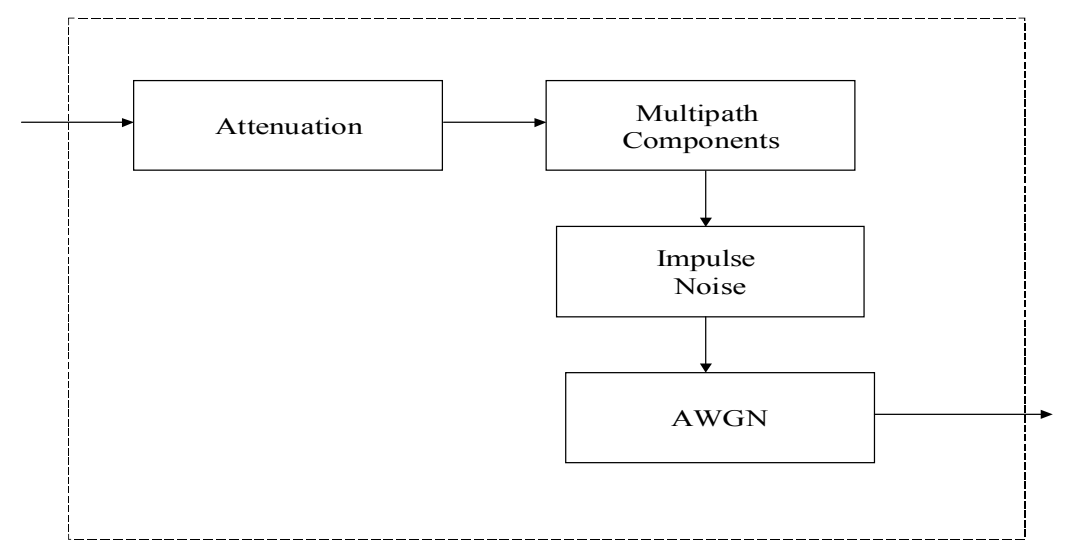

Figure 5.5: Power Line Channel Model

line channel.

Residential lines, on the other hand, offer a very hostile channel for data transportation [42]. A complete model for residential power line would include features like signal attenuation, background noise that can be modeled as AWGN, additive impulse noise, fading and multipath components of the signal. As stated earlier, signal attenuation is dependent on the physical seperation between the transmitter and the receiver $d$, and on the frequency of the signal $f_{o}$. The background noise, modeled as AWGN, is primarily because of interference from sources such as radio \& TV broadcasts, cosmic radiation, and thermal noise in the receiver circuitry. Noise in the form of impulsive and non-impulsive noise is an important factor, which should be included in the modeling of residential power line channel.

Fig. 5.5 represents a model of a power line channel that can be used to study and evaluate the residential power line channel. This model is partially derived from the echo model as presented in [33]. This model can be a useful tool for analysis of communication techniques like spread spectrum (SS) and orthogonal frequency division multiplexing (OFDM) that are being currently used for power line communications. An interesting technique for generating impulse noise is provided in [33]. The computer, for the discrete case, can generate AWGN random variables. Most of the noise explained in the previous section, 
including the impulse noise, can be characterized to a good extent by modeling them as AWGN by the application of the central limit theorem. The model also represents the multipath components of the signal that exist due to the presence of complex loads or improper terminations in the communication system, thereby leading to signal reflections.

The impulse response of the power line channel can be summarized as

$$
h(t)=A\left(d, f_{o}\right) \sum_{i=1}^{N} c_{i} \delta\left(t-\tau_{i}\right)
$$

The nature of the channel is such that narrowband signals will be highly attenuated (attenuation is on an average of $20 \mathrm{~dB}$ ) and those signals that remain will be highly distorted by the fading nature of the channel, such that it becomes highly impossible to recover the data that was being transmitted. To cope with such a channel, there is a need for a powerful transmission signal that will be able to overcome the frequency-selective fading and the attenuation, and still have sufficient energy, from which the information can be retrieved. This is where spread spectrum techniques are useful, primarily due to the wideband nature of the spread signals. Techniques like OFDM can also be used, as it mitigates the effects of frequency-selective fading and interference due to the presence of multipath components.

\subsection{Conclusion}

Power line communications offers exciting opportunities for future communication technologies. Utilities can benefit by investing in this technique, as it offers an economical alternative to other expensive communication technology being used presently. The channel model designed will provide a useful tool for the analysis of power line communications. 


\section{Chapter 6}

\section{Modulation and Coding for PLC}

\subsection{Introduction}

The power line communication channel, like the wireless channel, provides a very hostile medium for data communications. Methods like spread spectrum (SS) and orthogonal frequency division multiplexing (OFDM) have to be adopted to mitigate the effects of such a hostile channel and enable multiple users to access the channel at the same time.

Code division multiplex access (CDMA), which is based on SS technology, is used by the wireless industry to enable multiple users to access the entire allocated bandwidth at the same time [43]. CDMA allocates a unique spreading code to each individual user thereby enabling multiple access due to the uniqueness of the code. These spreading sequences also have very good cross correlation properties, thereby ensuring minimal interference from other users. On the other hand, OFDM involves the transportation of data bits on multiple frequencies that are orthogonal to each other. OFDM is a very effective technique for combatting the effects of a frequency selective fading channel and multipath propagation. Error correction codes like convolutional codes and Reed-Solomon codes can be incorporated into the system to improve BER performances.

\subsection{The Multi-Access Channel}

Three primary methods are used to enable multiple-access [44]: 


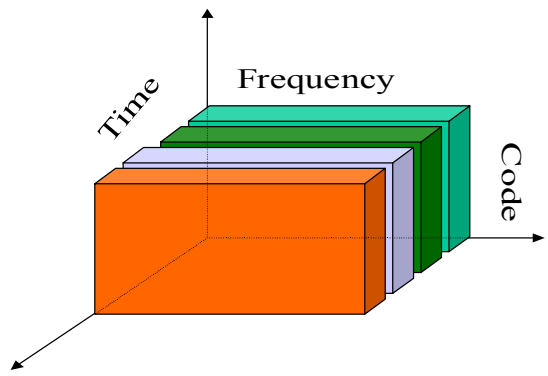

Figure 6.1: TDMA

- TDMA: In a time division multiplex access (TDMA) system, the radio spectrum is divided into time slots and in each slot, only one user is allowed to either transmit or receive. As shown in Fig. 6.1, each user is allocated a cyclically repeating time slot. Each TDMA frame comprises of $N$ such time slots, where each slot includes overhead such as a preamble, tail bits, and guard times between each user. The European GSM mobile system uses TDMA for mulitaccess. GSM uses $25 \mathrm{MHz}$ for each of the forward and reverse links, which is broken up into radio channels of $200 \mathrm{kHz}$ and each radio channel supports a total of 8 speech channels [45].

- FDMA: In a frequency division multiple access (FDMA) system, each user is allocated a unique frequency band from the available spectrum, as shown in Fig. 6.2. Since FDMA is a continuous-time transmission system as compared to TDMA, fewer overhead bits are needed for the purpose of synchronization and framing. This translates to higher efficiency for FDMA systems. On the other hand, FDMA systems are expensive due to the use of duplexers as both the transmitter and the receiver normally operate at the same time. Guard bands are allocated between users to minimize the effects of adjacent channel interference (ACI), which is caused due to the non-linearities of the electronic devices present in the FDMA system and due to the doppler effect whereby relative motion between the transmitter and the receiver results in a slight shift of the carrier frequency. The original US analog cellular system, known as the advanced mobile phone system (AMPS), is based on FDMA. 


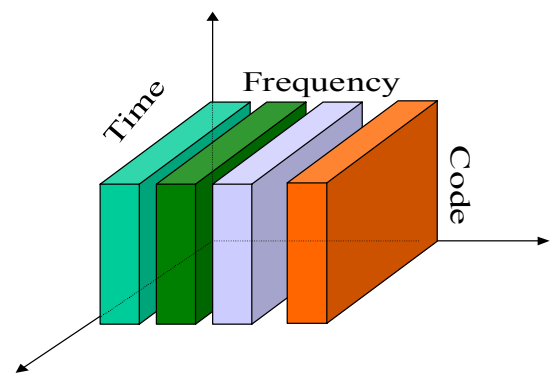

Figure 6.2: FDMA

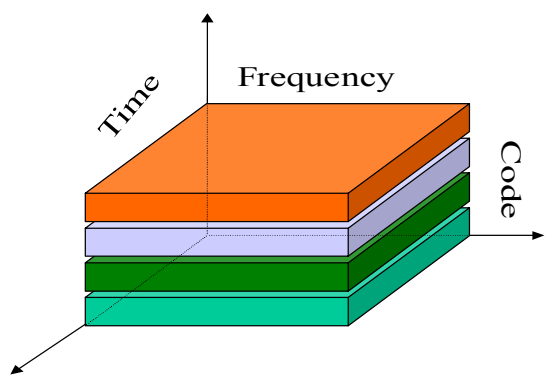

Figure 6.3: CDMA

- CDMA: In code division multiple access (CDMA) systems, the narrowband message signal is transformed into a wideband signal by one of two ways, direct sequence (DS) or frequency hopping $(\mathrm{FH})$. In a DS-SS system the narrowband message signal is multiplied by a binary wideband pseudorandom signal (or code). The result of this multiplication is a wideband signal with low power spectral density. As shown in Fig. 6.3 , each user is allocated a unique $\mathrm{PN}$ code which enables multiple users to transmit and receive at the same time and in the same frequency band. The PN codes have very low cross correlation which enables minimum interference between the users of the system. In FH-SS systems, using a frequency synthesizer, the carrier frequency of 
the modulated message signal changes continously in a predetermined pattern which is known to both the transmitter and the receiver. Pseudorandom codes fed into the memory of the frequency synthesizer determine the carrier frequency. Due to the limited advantages of FH-SS systems, DS-SS systems are more commonly used in practical applications ${ }^{1}$

\subsection{Spread Spectrum Systems}

In a DS-SS system, the narrowband message signal of the user is multiplied by a predetermined broadband signal called the spreading sequence or the PN code, as shown in Fig. 6.4. The PN code is normally implemented as a pseudo-random binary sequence clocked at a much higher rate than the user's message signal. In the DS-SS system, each data bit of the user is multipled by the PN code and then sent through the channel. At the receiver, the received signal is correlated with the corresponding $\mathrm{PN}$ code in order to retreive the user data. After the data is received, a threshold device is used to estimate the transmitted data.

DS-SS can be used to implement a CDMA system by assuring that any two users' PN codes have low cross correlation defined by [46]

$$
\rho_{j k}=\int_{0}^{T_{b}} s_{j}(t) s_{k}(t) d t \approx 0
$$

where $s_{j}$ and $s_{k}$ are the PN codes of users $j$ and $k$, and $T_{b}$ is the bit period. This ensures minimum interference among users that access the channel. It is this specific property of the spreading sequence which is primarily responsible for determining the soft capacity of a CDMA system.

The processing gain $G$ gives an indication of the bandwidth spreading of the user signal and is defined as the ratio of the bandwidth of the broadband signal to the bandwidth of the narrowband data signal, before the spreading [47]:

$$
G=\frac{T_{b}}{T_{c}}
$$

\footnotetext{
${ }^{1}$ http://www.oreilly.com
} 


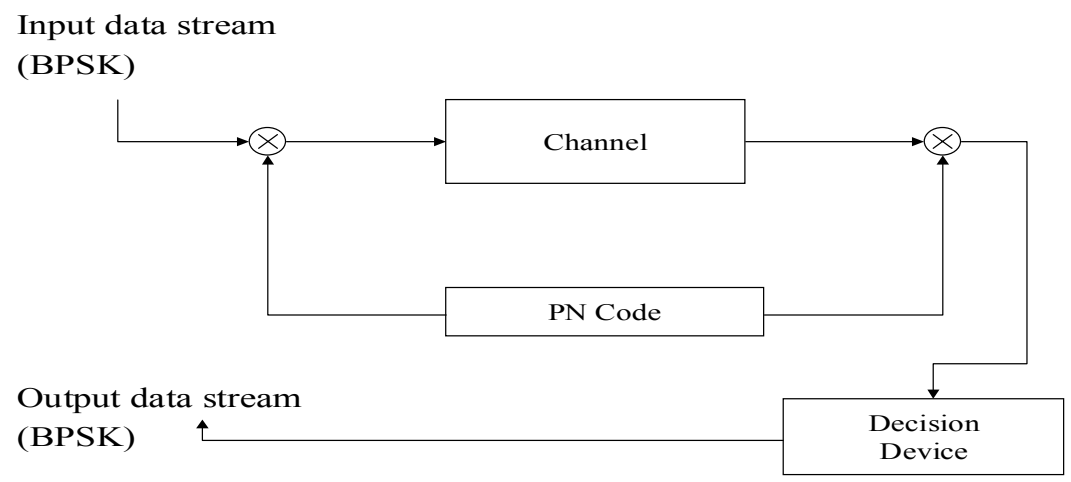

Figure 6.4: DS-SS block diagram

where $G$ is the processing gain, $T_{b}$ is the bit period of the message signal, and $T_{c}$ is the duration of a single element or "chip" of the PN code. Systems with higher processing gain can support more multiple-access users. Even single-user systems can benefit from a high value of $G$, as more "self-interference" caused by multipath reflections can be tolerated.

\subsubsection{Basic Synchronous CDMA Model}

As shown in Fig. 6.5, the synchronous CDMA model assumes that all the users in the system transmit at the same time such that their data symbols are perfectly aligned with one another [48]. This assumption is valid in the case of the downlink of a cellular system, that is from the base station to the individual mobiles. Synchronous CDMA concepts could also be applied to PLC as a method for combining multiple data messages into a single wideband signal. In a PLC system, the assumption of a synchronous CDMA model would be valid for data transmissions from the base unit or server to the individual PLC installations. At the receiver, matched filters are used, each matched to the signature waveform of a different user as indicated by Fig. 6.6

For an additive white Gaussian noise (AWGN) channel, the output of the $k^{\text {th }}$ user's 


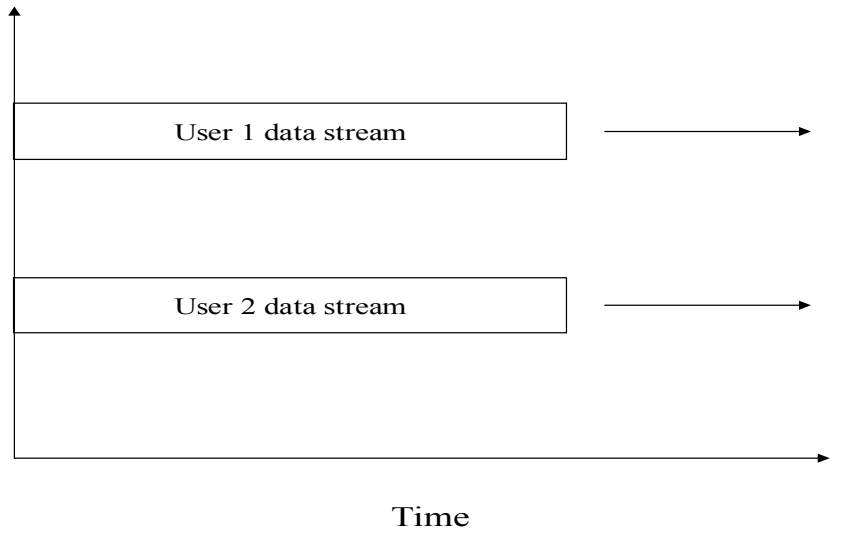

Figure 6.5: Synchronous CDMA

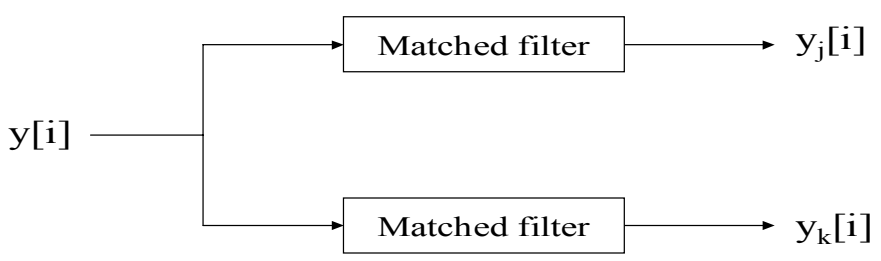

Figure 6.6: Synchronous CDMA - receiver end 
matched filter can be expressed as [49]

$$
y_{k}=A_{k} b_{k}+n_{k}+\sum_{j=1, j \neq k}^{K} A_{j} b_{j} \rho_{j k}
$$

where $b_{k} \in\{-1,+1\}$ is the bit transmitted by the $k^{t h}$ user and $A_{k}$ is the amplitude of the $k^{\text {th }}$ user, $n_{k}$ is the additive white Gaussian noise that models thermal noise plus other noise sources unrelated to the interfering signals and $K$ is the total number of users in the system.

It is convenient to express the above equation in vector form as

$$
\mathbf{y}=\mathbf{R A b}+\mathbf{n}
$$

where $\mathbf{R}$ is the normalized cross correlation matrix, $\mathbf{R}=\left\{\rho_{i j}\right\}$ with diagonal elements equal to one. Also, $\mathbf{y}=\left[y_{1}, \ldots, y_{K}\right]^{T}, \mathbf{b}=\left[b_{1}, \ldots, b_{K}\right]^{T}, \mathbf{A}=\operatorname{diag}\left\{A_{1}, \ldots, A_{K}\right\}$ and $\mathbf{n}$ is the zero mean gaussian random vector with covariance matrix equal to $\mathrm{E}\left[n n^{T}\right]=\sigma^{2} \mathbf{R}=\left(\frac{N_{o}}{2}\right) \mathbf{R}$ where $N_{o}$ is the two-sided noise spectral density.

For a quasi-static Rayleigh fading channel, the output of the $k^{\text {th }}$ user's matched filter can be expressed as

$$
y_{k}=\alpha_{k} A_{k} b_{k}+n_{k}+\sum_{j=1, j \neq k}^{K} \alpha_{j} A_{j} b_{j} \rho_{j k}
$$

where $\alpha_{k}$ and $\alpha_{j}$ are the fading channel coefficients. Expressing the above equation in matrix form,

$$
\mathbf{y}=\mathbf{R} \overline{\mathbf{A}} \mathbf{b}+\mathbf{n}
$$

where $\overline{\mathbf{A}}=\left[\alpha_{1} A_{1}, \ldots, \alpha_{K} A_{K}\right]$.

\subsubsection{Basic Asynchronous CDMA Model}

In the asynchronous CDMA model, the bit epochs of the users are not aligned with each other. This is the case in a cellular uplink, that is the link from the individual mobiles to the base station [48] [50]. Asynchronous CDMA could also be used in a PLC environment as a way to implement multiple-access. In a PLC system, the assumption of an 


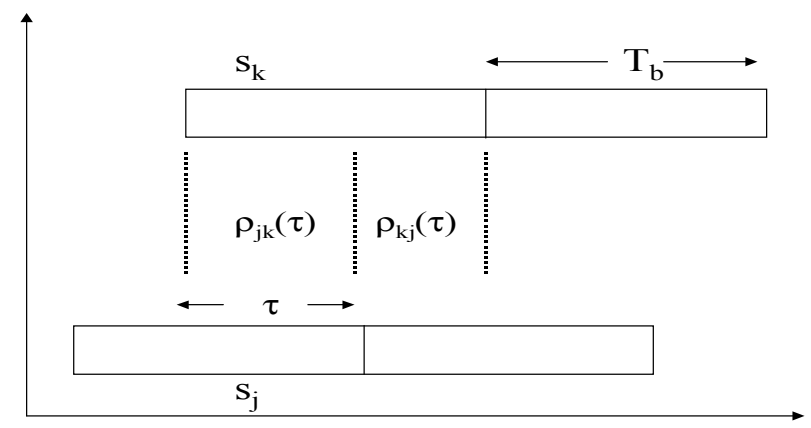

Figure 6.7: Asynchronous CDMA

asynchronous model would be valid for data transmissions from individual PLC installations to the base unit or server.

In simulating the asyncronous case, random time delays are introduced in order to model the misalignment of the bit epochs of the individual users. The random time delays are introduced such that $\tau \in\left[0, T_{b}\right]$.

In asynchronous CDMA, due to the lack of synchronism, the desired user may receive interference due to two bits from each interfering user and hence two types of cross correlations are defined between every pair of signature waveforms [48]. These correlations depend on the time offset $\tau$ between the two signals. From Fig. 6.7, where $k<l$, the correlations are defined as

$$
\rho_{j k}(\tau)=\int_{0}^{\tau} s_{k}(t) s_{j}\left(t+T_{b}-\tau\right) d t
$$

and

$$
\rho_{k j}(\tau)=\int_{\tau}^{T_{b}} s_{k}(t) s_{j}(t-\tau) d t
$$

For an AWGN channel, the signal at the output of the matched filter, $y_{k}$, can be expressed as 


$$
y_{k}^{(i)}=b_{k}^{(i)}+n_{k}+\sum_{j=1, j \neq k}^{K}\left(\rho_{j k} b_{j}^{(i)}+\rho_{k j} b_{j}^{(i+1)}\right)
$$

where $y_{k}^{(i)}$ is the $i^{t h}$ bit of the output of the $k^{t h}$ user, $b_{k}^{(i)}$ is the desired user's

current bit, $b_{j}^{(i)}$ is the $j^{\text {th }}$ interfering user's current bit, $b_{j}^{(i+1)}$ is the $j^{\text {th }}$ interfering user's next bit, and $n$ is the additive white Gaussian noise.

Similarly for a two user Rayleigh fading channel, the output of the matched filter can be expressed as

$$
y_{k}^{(i)}=\alpha_{k}^{(i)} b_{k}^{(i)}+n_{k}+\sum_{j=1, j \neq k}^{K}\left(\alpha_{j}^{(i)} \rho_{j k} b_{j}^{(i)}+\alpha_{j}^{(i+1)} \rho_{k j} b_{j}^{(i+1)}\right)
$$

where $\alpha_{k}^{(i)}$ and $\alpha_{j}^{(i)}$ are the fading channel coefficients during the $i^{\text {th }}$ signaling interval of the $k^{\text {th }}$ and $j^{\text {th }}$ user respectively.

\subsubsection{The Spreading Sequence}

The spreading sequence is an important aspect of any SS system design. Codes are generated using shift registers with feedback. Spreading techniques like Gold and Kasami sequences can ensure that the signature waveforms have very good auto correlation and cross correlation properties, that is [51]

$$
\frac{1}{N} \sum_{n=0}^{N-1} s_{j, n} s_{j, n+k} \approx 0 ; \text { for } k \neq 0
$$

and

$$
\frac{1}{N} \sum_{n=0}^{N-1} s_{j, n} s_{k, n+k} \approx 0 ; \text { for all } k \text { and } i \neq j
$$

respectively, where $s_{j, n}$ denotes the $n^{\text {th }}$ chip of the PN sequence of the $j^{\text {th }}$ user, and $s_{k, n+l}$ denotes the $(n+l)^{t h}$ chip of the $k^{t h}$ user.

Certain properties of the PN code are as follows [52]: 
1. Increasing the value of the processing gain $G$ reduces both multiuser-interference and self-interference proportionally.

2. Increasing $G$ reduces the synchronization error, thereby increasing the reliability of estimates of the signal's time of arrival.

3. Increasing $G$ proportionally reduces the performance degradation caused by a narrowband jammer. More specifically, when a system is operated in a jamming environment, the bit error probability for unspread BPSK signaling, in the absence of thermal noise, can be expressed as

$$
P_{e}=\rho Q\left(\sqrt{\frac{2 E_{b} \rho}{N_{j}}}\right)
$$

where $\rho$ is the pulse duty factor of the jammer, $E_{b}$ is the energy per bit, $N_{j}$ is the one-sided average jammer power spectral density and is given by

$$
N_{j}=\frac{J}{W}
$$

where $J$ is the average power of the jamming signal and $W$ is the one-sided transmission bandwidth. Spread spectrum can be used to reduce the jamming signal interference. If an SS signal of processing gain $G$ is used, the BER, in the presence of the jamming signal, reduces to

$$
\hat{P}_{e}=\rho Q\left(\sqrt{\frac{2 E_{b} \rho}{G N_{j}}}\right)
$$

\subsubsection{Simulation Results}

Simulations were performed to determine the performance of asynchronous and synchronous CDMA systems. For the purpose of simulations, randomly generated codes have been used. Fig. 6.8 and Fig. 6.9 indicate the BER performance of a two user BPSKmodulated CDMA system, in comparison to a single-user unspread BPSK system, both in 


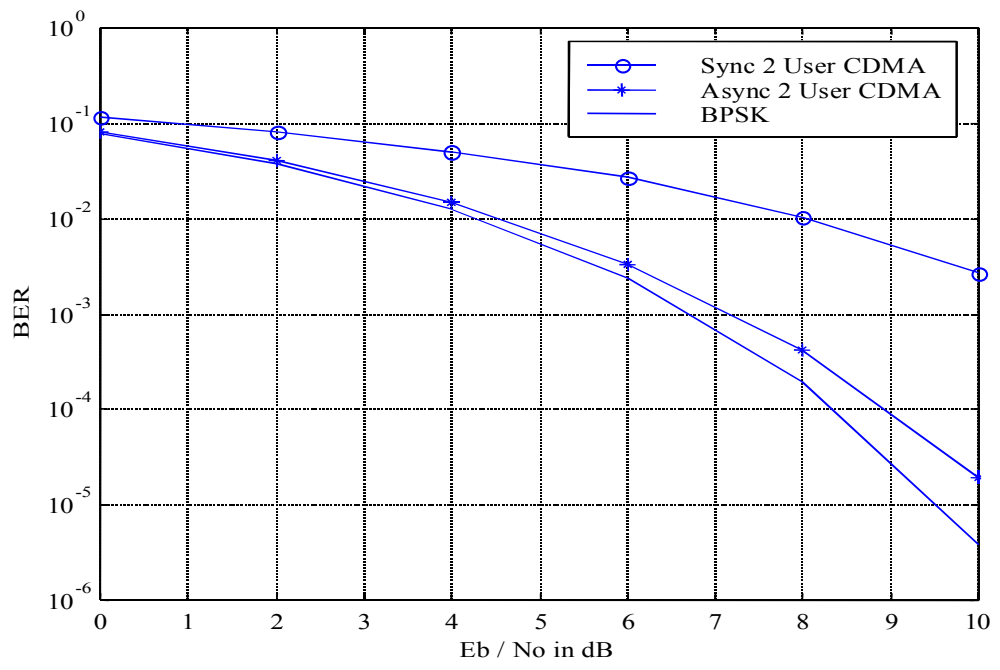

Figure 6.8: Performance of synchronous and asynchronous CDMA in an AWGN channel, with $\mathrm{G}=7$

an AWGN channel and a Rayleigh fading channel, respectively.

The randomly chosen spreading sequence had a processing gain $G$ of 7 . The fading channel is considered to be quasi-stationary. From the graph, it is clear that asynchronous CDMA performs better than synchronous CDMA inspite of the fact that there are more interfering bits in the latter case. This can be explaind by simple probability. In the case of synchronous CDMA, each desired bit had only one interfering bit and the probability that the interfering bit is out of phase with the desired bit is 0.5 since the binary signal is considered to be equiprobable. In the case of asynchronous CDMA, each desired bit has two interfering bits and the probability that both the interfering bits are out of phase with the desired bit is less than 0.5. Hence, constructive interference for an asynchronous CDMA system helps the system to achieve better BER performance as compared with synchronous CDMA systems. 


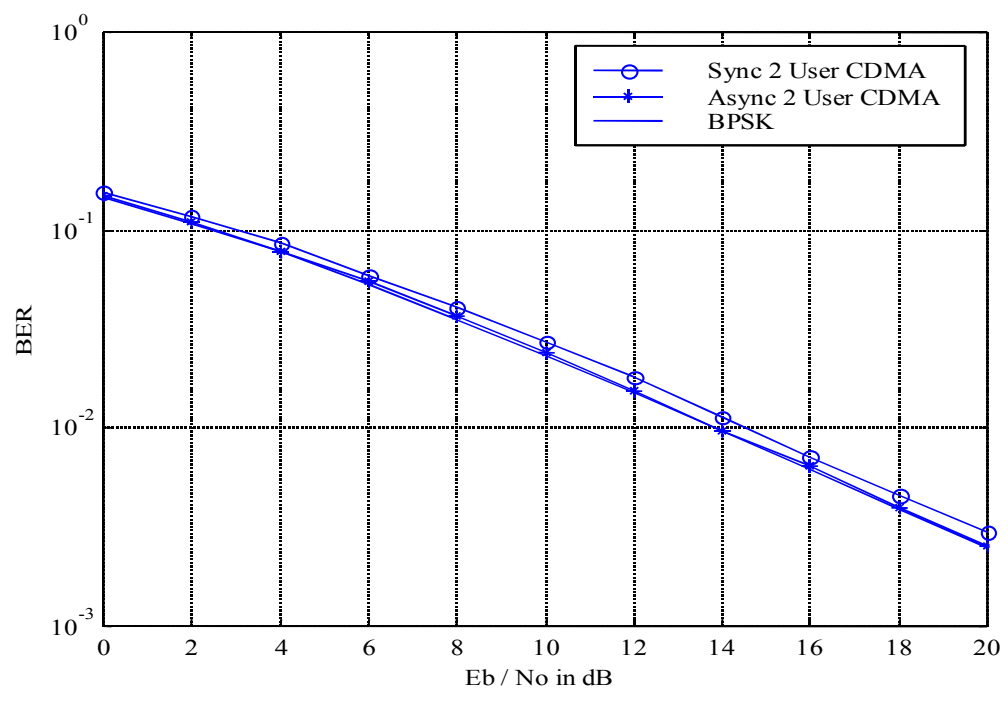

Figure 6.9: Performance of synchronous and asynchronous CDMA in a flat fading Rayleigh channel, with $\mathrm{G}=7$

\subsection{Rake Receivers}

\subsubsection{Introduction to RAKE Receivers}

Multipath propagation is the most important factor limiting mobile and portable radio communications [53]. In a mobile environment, the signal is reflected and scattered due to obstacles along the propagation path. The multipath components suffer from amplitude fluctuations and phase shifts and arrive at the receiver with different time delays [54]. Conventional receivers extract information from only a single component of the transmitted signal. Rake receivers, first introduced by Price and Green, combine the energy from several multipath components in order to extract the required information [55].

An $M$-finger rake receiver consists of $M$ correlation receivers that are tuned to $M$ distinct multipath components of the transmitted signal [56]. Assume that a transmitted signal suffers from multipath fading and that a two finger rake receiver is used to detect the multipath components. The first signal component arrives at $\tau_{1}=0$ and the second component arrives at $\tau_{2}$. The first finger of the rake receiver is correlated with the first 


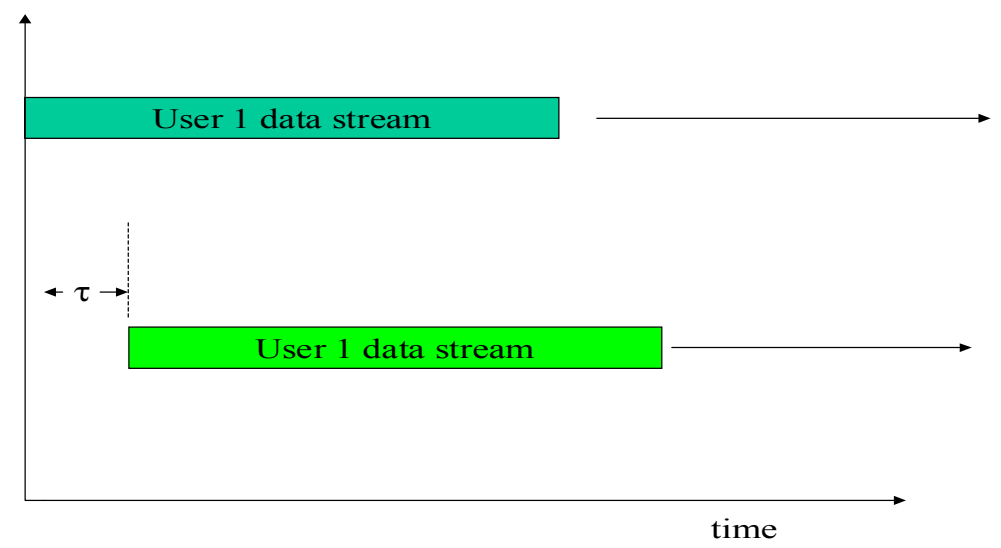

Figure 6.10: Multipath propagation, single user, 2 multipath components

arriving signal while the second finger is correlated with the second component. Fig. 6.11 indicates the model of a two finger rake receiver where $w_{1}$ and $w_{2}$ are the weights that are used to multiply the signal passing through the respective finger of the rake receiver.

In a mobile channel, rake receivers are present both at the base station and in the mobile unit of each individual user. In the present CDMA systems (developed by Qualcomm and called IS-95, or cdma ONE), the base station has a four-finger rake receiver and the mobile unit has a three-finger rake receiver. The number of fingers is chosen based on the multipath nature of the channel and economic considerations. Rake receivers can also be implemented for PLC, both at the base or server unit and at indvividual PLC installations.

\subsubsection{Simulation Results}

Simulations were performed for implementing the rake receiver and the following assumptions were made:

1. There are two distinct multipath components and therefore two fingers are adequate to provide the necessary information. 


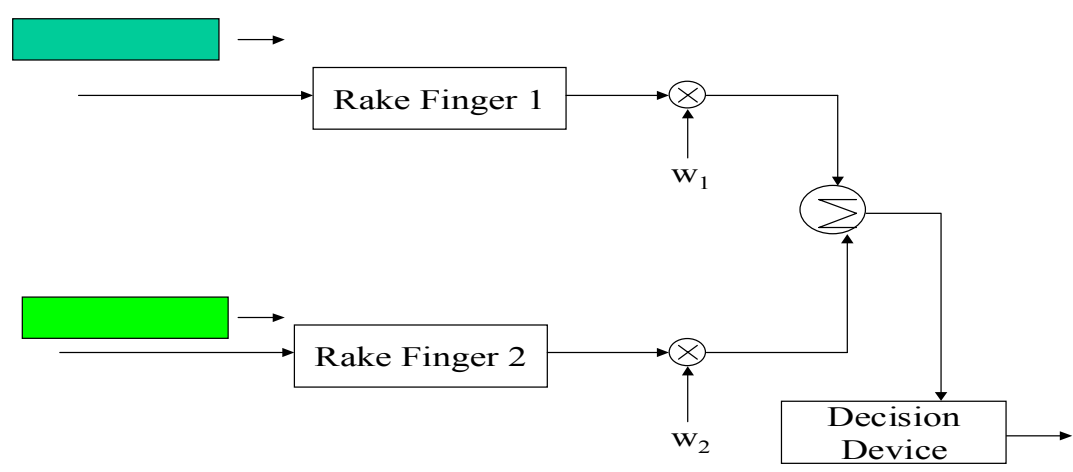

Figure 6.11: Rake receiver, 2 fingers

2. The fingers were tuned to the multipath components exactly.

3. The multipath components do not suffer from any phase shifts, although this is not necessarily the case in a practical mobile or PLC channel.

4. The channel is assumed to be quasi-static and Rayleigh fading.

5. The multipath components of each signal are not independent from each other, but rather interfere with each other.

The detected components are combined to ensure that maximum information is extracted from the multipath components. The decision estimates from each finger are weighted by a weighting function before they are combined. For the two finger rake receiver, the decision static was calculated in the following manner:

1. Let $z_{i, k}^{(1)}$ be the output of the first finger's correlator corresponding to the $i^{t h}$ bit of the $k^{\text {th }}$ user. 
2. Let $z_{i, k}^{(2)}$ be the output of the second finger's correlator corresponding to the $i^{\text {th }}$ bit of the $k^{\text {th }}$ user.

3. The final bit estimate is provided by

$$
\hat{z}_{i, k}=z_{i, k}^{(1)}\left|z_{i, k}^{(1)}\right|+z_{i, k}^{(2)}\left|z_{i, k}^{(2)}\right|
$$

4. The decision device estimates the bit by

$$
\hat{y}_{i, k}=\operatorname{sgn}\left(\hat{z}_{i, k}\right)
$$

The model used to estimate the bit is equivalent to maximal ratio combining (MRC) of the signals. More confidence is placed on a stronger correlation peak that indicates the presence of the desired signal. A small correlation product is weighted less in order to reduce the probability of making a faulty decision. The theoretical bit error probability for MRC combining and BPSK signalling, $P_{b}$, is given by

$$
P_{b}=\left(\frac{1-\nu}{2}\right)^{L} \sum_{k=0}^{L-1}\left(\begin{array}{c}
L-1+k \\
k
\end{array}\right)\left(\frac{1+\nu}{2}\right)^{k}
$$

where

$$
\nu=\sqrt{\frac{S N R}{1+S N R}}
$$

Fig. 6.12 indicates the BER performance of a rake receiver with two fingers, for a single-user system, in comparison to the theoretical bound given by Eqn. (6.18). The processing gain used in this simulation is 7 . The channel is assumed to be quasi-static and frequency-selective Rayleigh fading. The single user in the system is assumed to have two multipath components. The graphs clearly indicate that rake receivers help improve performance even in the presence of multipath components of the signal (self-interference). The plot also indicates that more the number of finger, better will be the performance of the spread spectrum system, in a frequency-selective channel.

Rake receivers are a useful tool for extracting information from multipath components, over any given medium of communication. Forward error correcting codes like the 
convolutional code or the Reed-Solomon coding can be used to improve BER performance of the system tremendously. Trellis based Viterbi decoders, in case of convolutional codes, can be used at the receiver end to decode the received data that has been corrupted by the channel.

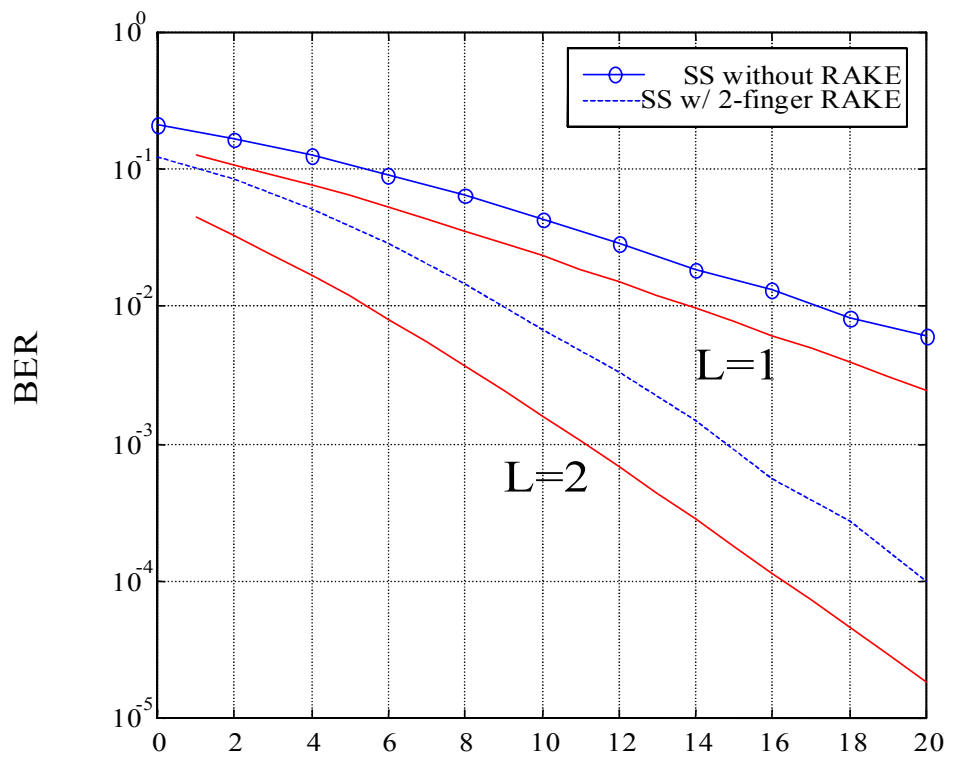

$\mathrm{Eb} / \mathrm{No}$ in $\mathrm{dB}$

Figure 6.12: Rake receiver performance in comparison with theoretical bounds for single user in Rayleigh fading channel, $\mathrm{G}=7$ 


\subsection{Convolutional Coding}

\subsubsection{Introduction to Convolutional Coding}

Channel coding involves adding redundant parity bits to the data bits before transmission through the communication channel. Convolutional coding is one type of channel coding. A convolutional code is generated by passing the user data signal through a sequence of shift registers which forms a linear finite-state machine. In general, the shift register consists of $K_{c}(k-$ bit) stages and $n$ function generators [57]. The input data to the encoder, which is binary data, is shifted into and along the shift register $k$ bits at a time.

The number of output bits for each $k$ bit input sequence is $n$ bits. The code rate $r$ is defined as the ratio of $k$ to $n$ [55],

$$
r=\frac{k}{n}
$$

The parameter $K_{c}$ is called the constraint length of the code. The performance of a convolutional code depends on the rate of the code and the constraint length. A large constraint length leads to a more robust code, but at the same time, increases the decoder complexity and decoding delay exponentially.

A convolutional code can be defined by its generator matrix. Fig. 6.13 indicates a

rate $r=\frac{1}{2}$ convolutional code with generator matrix $G=[101,111]$, which can be written in octal as $(5,7)$. The rate of this code is $\frac{1}{2}$, since there are two output bits present for every input bit and the constraint length is $K_{c}=3$, since each output bit depends on up to three bits (the current input bit plus the two bits stored in the shift register).

Decoding of the convolutional code is performed by the Viterbi Decoder, which is the optimal solution to finding the maximum likelihood sequence of data bits that were transmitted [57]. A description of the Viterbi decoder can be found in detail in [52].

\subsubsection{Simulation Results}

The generator matrix used for the purpose of simulation is given by

$$
g=[1111001,1011011]
$$




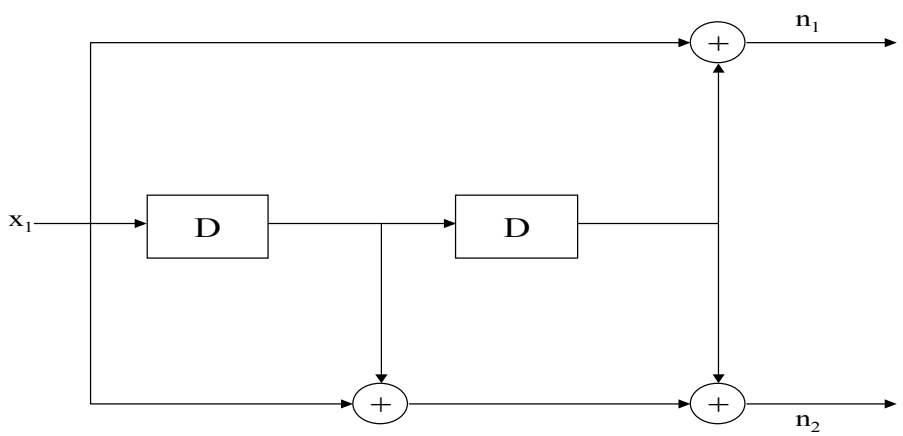

Figure 6.13: Convolutional encoder

The rate of this convolutional code is $r=\frac{1}{2}$ and the constraint length $K_{c}$ is 7 . Fig. 6.14 indicates the performance of a convolutionally coded 2 user BPSK-modulated CDMA system. Fig. 6.15 indicates the BER performance of the encoded data, for a rake receiver, and a synchronous model. Fig. 6.16 indicates the BER performance of the convolutionally encoded data, for a rake receiver, and an asynchronous model. The processing gain for both the simulations $G$ is 7 . The channel is assumed to be quasi-static, Rayleigh fading, and frequency-selective in nature. There are two multipath components for each user's signal. The BER performances of the coded data are compared with uncoded single-user unspread BPSK-modulated signal with no multipath components, which indicate the coding gain of the system using convolutional coding and Viterbi decoding. 


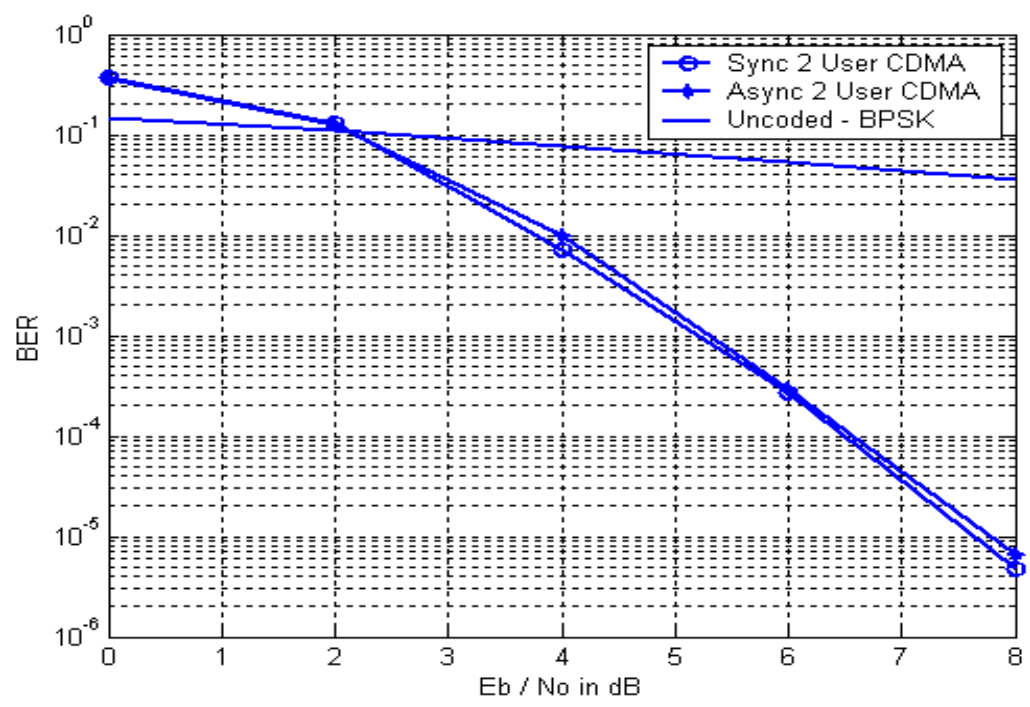

Figure 6.14: Performance of coded CDMA - convolutional code in Rayleigh fading channel, rate $=\frac{1}{2}, G=7$

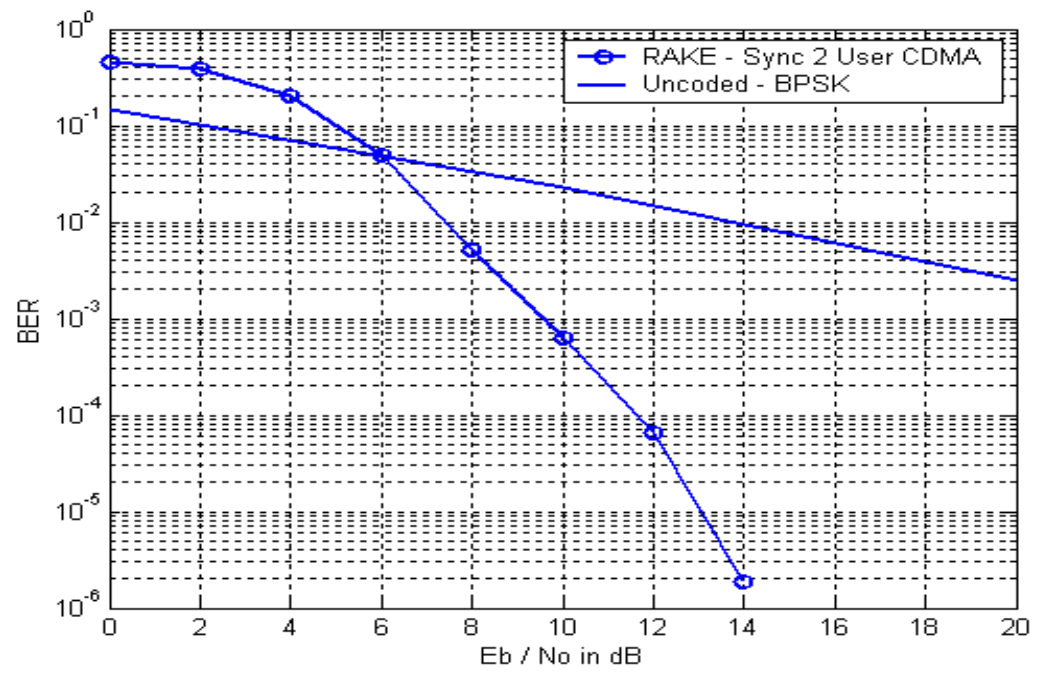

Figure 6.15: Rake receiver performance - synchronous model - convolutional code, in a Rayleigh fading channel, rate $=\frac{1}{2}, G=7$ 


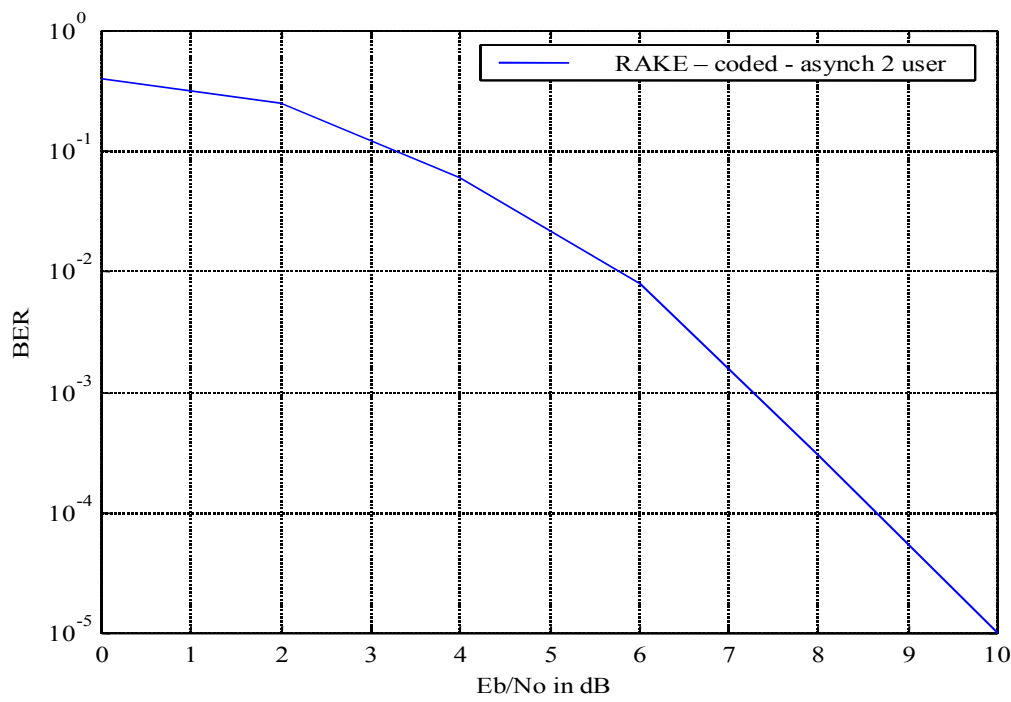

Figure 6.16: Rake receiver performance - asynchronous model - convolutional code, in a Rayleigh fading channel, rate $=\frac{1}{2}, G=7$

\subsection{OFDM}

\subsubsection{Introduction to OFDM}

Orthogonal frequency division multiplexing (OFDM) is an effective technique for mitigating the effects of multipath propagation. OFDM reduces the effects of inter-symbol interference (ISI) by making the data block period much larger than the delay spread of the channel [58]. Fig. 6.17 represents an OFDM system. OFDM converts blocks of data into OFDM symbols using an N-point inverse fast Fourier transform (IFFT) to modulate them onto the subcarriers which are orthogonal to each other [59].

The subcarrier frequencies are separated by multiples of the inverse of the signaling interval $\frac{1}{T}$ so that, with no signal distortion in transmission, the coherent detection of a signal element in any one subchannel of the parallel system gives no output for a received element in any other subchannel due to the orthogonality of the subcarriers. Guard bands are placed between subcarrier bands in case there is a loss of orthogonality during transmission through the channel [60]. 


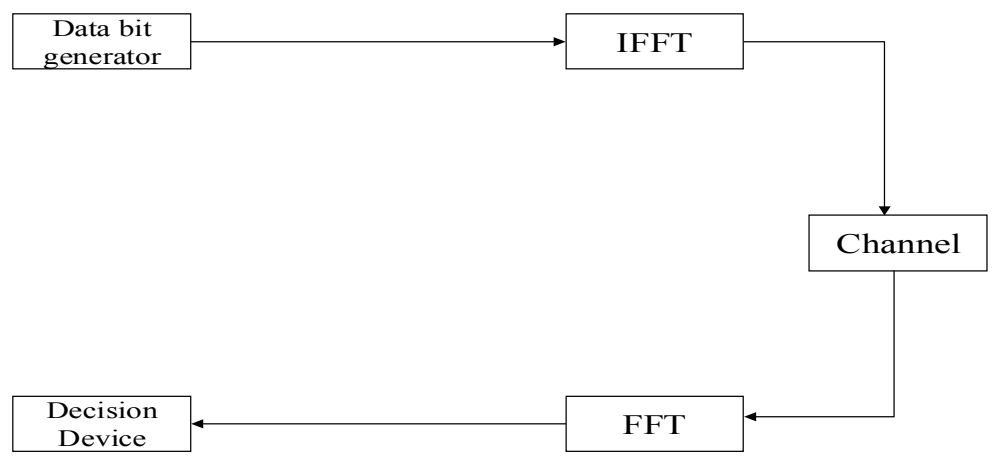

Figure 6.17: OFDM system

In an OFDM system, data is carried on narrowband subcarriers in the frequency domain. The data is transformed into the time-domain using an IFFT at the transmitter and transformed back to the frequency domain using a fast Fourier transform (FFT), at the receiver [61]. The total number of subcarriers defines the number of points of the FFT/IFFT, that is $N$ subcarriers would mean an $N$-point FFT/IFFT.

The discrete time representation of the signal after the IFFT is given as

$$
u_{n}=\frac{1}{\sqrt{N}} \sum_{k=-\frac{N}{2}}^{\frac{N}{2}-1} b_{k} e^{j 2 \pi \frac{k}{N} n}
$$

where $n \in\left[-\frac{N}{2}, \frac{N}{2}-1\right]$. At the receiver end, the data is recovered by performing a FFT on the received signal

$$
y_{k}=\frac{1}{\sqrt{N}} \sum_{n=-\frac{N}{2}}^{\frac{N}{2}-1} u_{n} e^{-j 2 \pi \frac{n}{N} k}
$$

where $k \in\left[-\frac{N}{2}, \frac{N}{2}-1\right]$.

In order for the orthogonality of OFDM to be preserved, the following must be true: 
1. The receiver and the transmitter must be perfectly synchronized. This means they both must assume exactly the same modulation frequency and the same time-scale for transmission (which usually is not the case).

2. The analog components used by both the transmitter and the receiver, must be of very high quality.

3. The subchannel must be flat faded.

In particular the last point is important, since OFDM is chosen to combat the multipath channel. The solution to this problem is that the OFDM symbols are artificially prolonged by periodically repeating the 'tail' of the symbol and preceding the symbol with it. At the receiver this guard interval is removed again.

\subsubsection{Simulation Results}

Simulations were performed to indicate the BER performances of an OFDM system in the presence of multipath components. Fig. 6.18 indicates the simulated performance of an OFDM system with 2 multipath components and 8 subcarriers, in comparison with BPSK signaling having no multipath components, over a frequency selective-channel channel. The graph indicates that OFDM performs well in a frequency-selective channel because of the narrowband nature of its symbols' orthogonal subcarriers. Fig. 6.19 indicates the coding gain of a simulated coded-OFDM with 2 multipath components, as compared to uncoded BPSK signaling without multipath components. The generator matrix used for the convolutional encoding of the data is the same as given by Eqn. (6.21). 


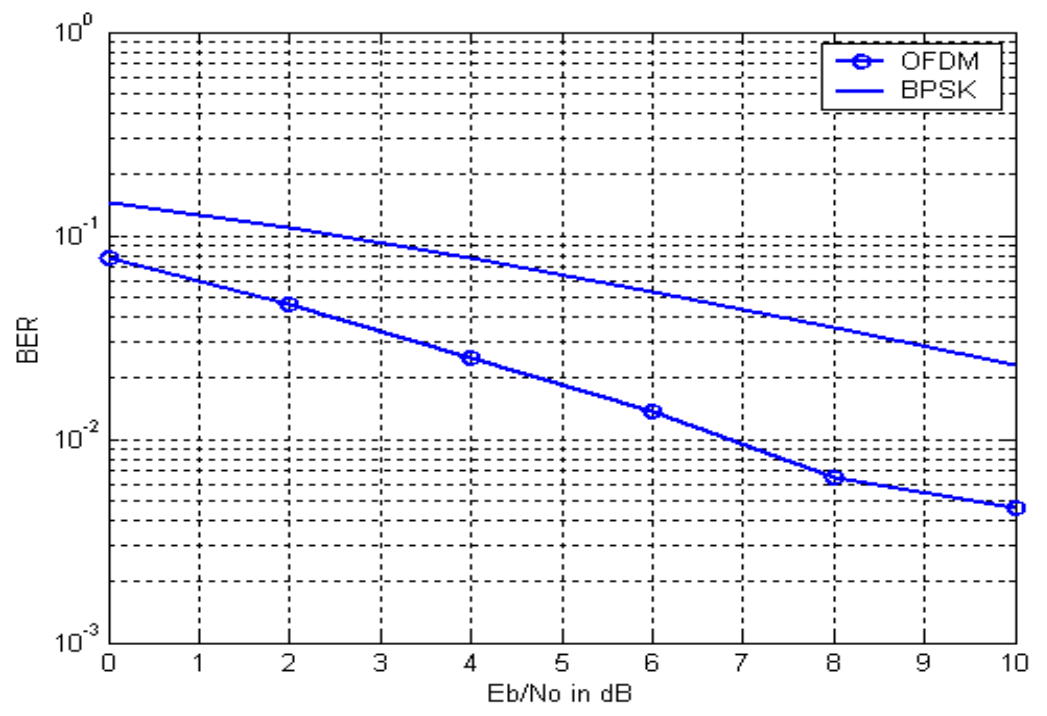

Figure 6.18: Comparison between uncoded-OFDM - 2 ray model - 8 subcarriers and singleuser BPSK signalling in Rayleigh fading channel

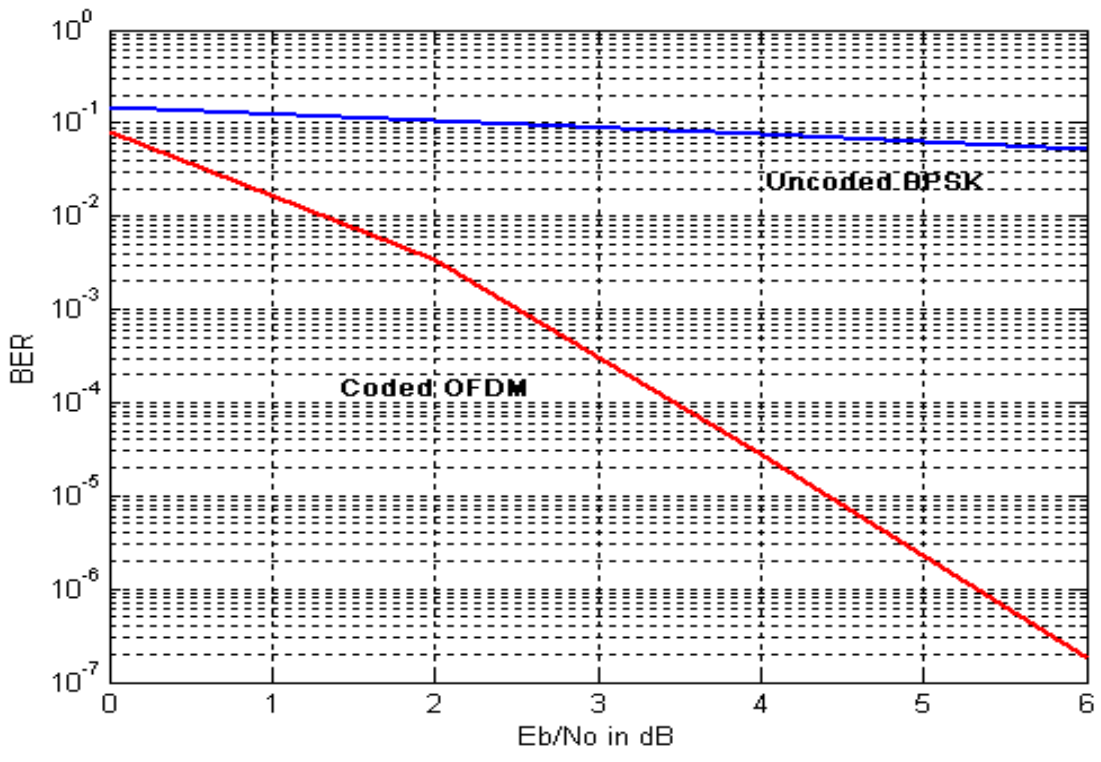

Figure 6.19: Convolutionally coded (rate $=\frac{1}{2}, G=7$ ) OFDM versus uncoded BPSK - 2 ray model in Rayleigh fading channel - 8 subcarriers 


\subsection{Conclusion}

This chapter introduced the concept of multiaccess techniques including TDMA, FDMA, and CDMA. DS-SS and OFDM are techniques which allow for reliable data transmission through hostile channels typical of the mobile and power line environments. Spread spectrum, which is the basis of CDMA, helps to mitigate the effects of narrowband interference and OFDM helps in mitigating the effects of multipath propagation. Channel coding of data can be used to drastically improve the BER performances of the communication system. Binary codes such as convolutional codes or non-binary block codes such as ReedSolomon codes can be used to improve performance at low SNR. The power line channel, in many ways, is like the wireless channel and techniques like SS and OFDM can be used for power line communications (PLC) as well. 


\section{Chapter 7}

\section{Power Line Communication System Design}

\section{$7.1 \quad$ Introduction}

In this chapter, we present an example system and protocol design suitable for power line communication systems. Such a design provides insight into the key issues of PLC, and an understanding of the implementation process. Multiple access in PLC can be implemented using either time division multiple access (TDMA) or carrier sense multiple access (CSMA) [42]. Each approach has its own advantages and disadvantages, which will be highlighted as the chapter progresses.

The discussion of an example protocol helps to explain what occurs in the network layers that are above the physical layer. The protocol design presented in this chapter is similar to the distributed network protocol (DNP 3.0) that is currently being used by utilities for communication between a remote terminal unit (RTU) and an intelligent electronic device (IED) in a supervisory control and data acquisition (SCADA) environment, and the CEBus protocol for home automation.

\subsection{PLC System Implementation}

Fig. 7.1 gives a block diagram of a system that can be used for PLC systems. The raw data is collected by the system and then processed by the data link layer into a 


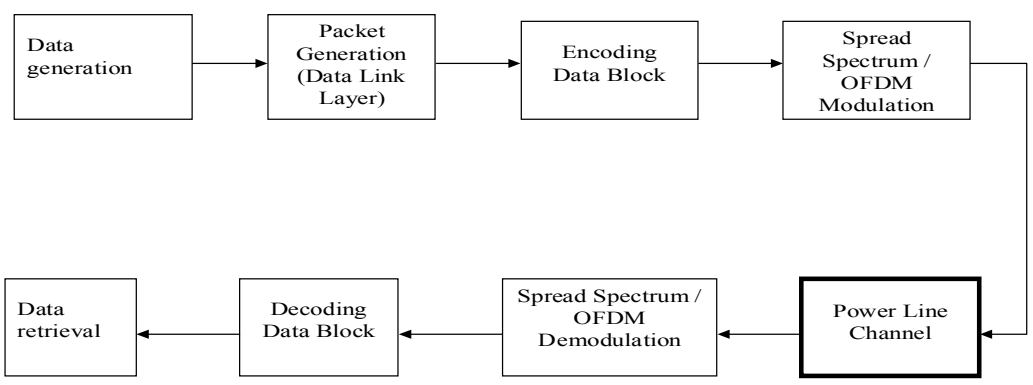

Figure 7.1: PLC system

packet that contains the source address and destination address, among other fields. The data link layer consists of two sublayers, namely the logical link and the medium access control (MAC) sublayers. The logical link sublayer assures a reliable point-to-point communication link through automatic repeat request (ARQ) while MAC sublayer supervises the channel in terms of who uses it and when (multiple-access). The MAC sublayer attaches the destination and source addresses to the protocol data unit (PDU), passed onto it by the logical link sublayer, and is also responsible for error detection of the packets. The logical link sublayer keeps a track of the sequencing of packets and is also responsible for retransmitting those packets that are detected to be in error by the MAC sublayer.

The PLC system can be designed and implemented using spread spectrum modulation or orthogonal frequency division multiplexing, that were discussed in detail, in Chapter 6. The packets can be FEC-encoded or uncoded, where a convolutional encoder could be used in the former case. In the case of convolutional encoding, Viterbi decoding can be used at the receiver end to decode the data. Reed-Solomon codes can also be used to FECencode the data. Finally, data retrieval can be performed at the receiver as indicated by Fig. 7.1. Regardless of whether or not FEC-encoding is used, the data must be encoded with an error detecting code, such as a cyclic redundancy check (CRC) code, which is used by the ARQ-protocol to determine when a retransmission is necessary. 


Source $\quad \longrightarrow$ Frame O

Figure 7.2: Stop-and-wait ARQ timing diagram

\subsection{Error-control}

Error control is an important aspect of any communication system [57]. It is important because data frames could be lost or damaged in transit. Automatic repeat request (ARQ) is a major part of the error control mechanism [62]. The source will transmit new packets after receiving a positive acknowledgment (ACK) signal from the destination, otherwise, it will retransmit the same packet if it receives a negative acknowledgment (NACK) from the destination, which indicates that the previous packet was not received correctly. There are three main categories of ARQ: Stop-and-wait, go-back-N, and selective-repeat $[63]$

\subsubsection{Stop-and-wait ARQ}

With stop-and-wait ARQ, the source transmits a data frame and then waits for a certain amount of time to receive a positive acknowledgment (ACK) or a negative acknoledgement (NACK) from the destination. No other data frame is allowed to be transmitted until the ACK / NACK is received. Fig. 7.2 shows the timing of stop-and-wait ARQ.

In the example shown in Fig. 7.2, the source transmits frame number 0. The destination, on receiving the correct data, replies by sending an ACK 1 indicating that it has received frame 0 and is waiting for the transmission of frame number 1 . In such an ARQ scheme, two types of errors are possible: The message received by the destination could be corrupted and hence it will not send the ACK signal. In this case, the source will wait for a certain amount of time and then retransmit the original frame. The destination could 
also send a NACK back to the source to alert it to the fact that the previous transmission attempt had failed. This way, the source does not need to wait until its internal timer expires to retransmit the packet, thereby improving overall throughput. The other error possibility is that the ACK signal might be lost in transit even though the destination has received the correct message. The source, not having received an ACK signal, will retransmit the original data frame after waiting for a stipulated amount of time. Hence, in this case, the destination now has two correct versions of the same data frame. Algorithms built into the protocol discard duplicate copies of the transmitted frame. Stop-and-wait ARQ is easy to implement but can be disadvantageous during high data volumes on the communication network, and when the network delay is long, because the source must sit idle while waiting for an ACK.

\subsubsection{Go-back-N ARQ}

A commonly used error control mechanism based on a sliding window is called go-back-N ARQ [63]. In go-back-N ARQ, the source sends a certain number of frames $\mathrm{N}$ without waiting for an acknowledgment from the destination. The number of unacknowledged frames transmitted depends on the sliding window size.

If there are no errors in the transmission, the destination sends a positive acknowledgment $(\mathrm{RR}=$ receive ready). If the destination detects an error in a certain frame, it will send a negative acknowledgment for that particular frame (REJ = reject). The destination will then discard that particular frame and all subsequent frames until the concerned frame in error is correctly received. Hence, when the source receives a REJ, it must not only retransmit the particular frame that was in error (for which the negative acknowledgment was received), but should also retransmit all the frames that were transmitted in the interim period. Fig. 7.3 indicates the timing of a go-back-N ARQ protocol.

In the go-back-N ARQ example shown in Fig. 7.3, the source transmits three frames $(0,1,2)$ according to the size of the sliding window $(\mathrm{N}=3)$ without waiting for an acknowledgment. The destination receives all the three frames correctly and sends RR 3 indicating that it is ready to receive the remaining frames. On receiving the $R R 3$, the source sends another three frames $(3,4,5)$. The destination detects an error in frame 4 and 


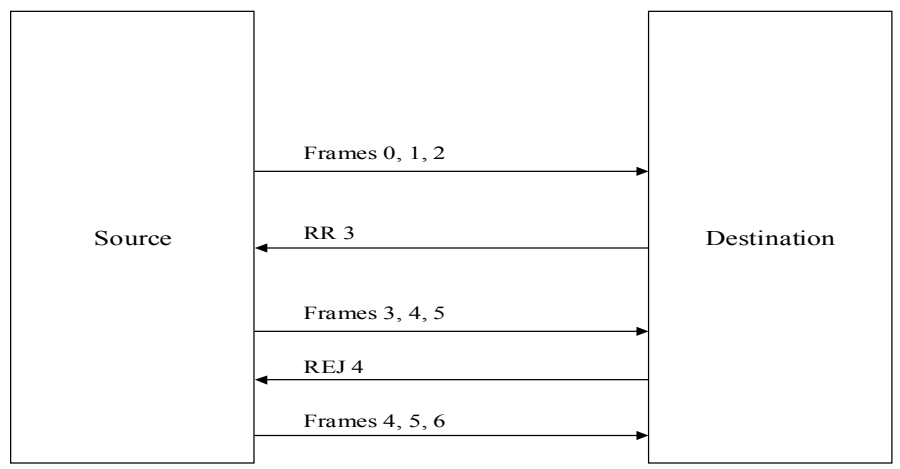

Figure 7.3: Go-back-N ARQ timing diagram

accordingly sends a negative acknowledgment for frame 4 (REJ 4). On receiving REJ 4, the source not only has to transmit frame 4 for which the negative acknowledgment was received but also frame 5 , which was sent during the interim period.

With a go-back-N ARQ protocol, there could be the possibility of a packet getting lost or the possibility of an RR or REJ signal getting lost, while in transit. There are three cases that could occur and are highlighted as follows [63]:

1. The source sends frame $K$, that is lost in transit, after which it sends frame $K+1$. The destination receives frame $\mathrm{K}+1$ out of order and sends a REJ $\mathrm{K}$ since it has not received frame $\mathrm{K}$. In such circumstances, the source has to retransmit frame $\mathrm{K}$ and all subsequent frames that were transmitted during the interim period.

2. The source sends frame $\mathrm{L}$ and does not have any more frames to send after that. Frame $\mathrm{L}$ is the last frame that needed to be transmitted. Now, if frame $\mathrm{L}$ is lost during transit, the source does not receive any positive acknowledgment from the destination for frame L. The source then signals the destination with a packet with a particular bit set to 1 . The destination receives this particular signal from the source which forces the destination to send an RR signal for the frame that it is expecting (RR L). The source then retransmits frame L. 


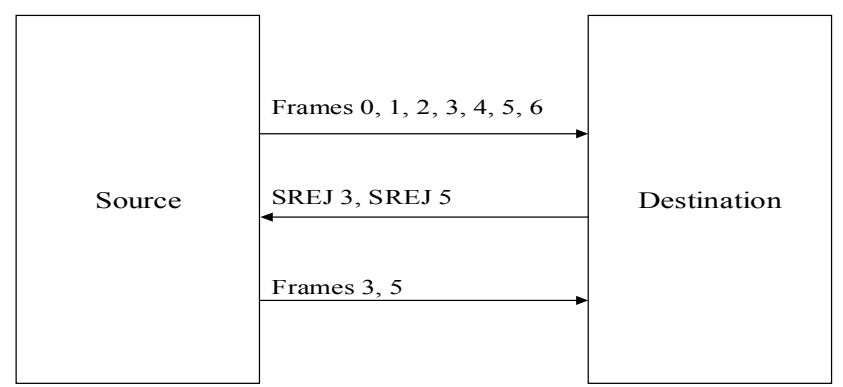

Figure 7.4: Selective-repeat ARQ timing diagram

3. In the case of lost RR or REJ, a similar procedure is adopted as was detailed for frame L transmission.

\subsubsection{Selective-repeat ARQ}

In this kind of an ARQ mechanism, only those frames are retransmitted for which a negative acknowledgment (SREJ) has been received. The source transmits $\mathrm{N}$ frames according to a window size without waiting for an acknowledgment. The destination detects errors in certain frames from the $\mathrm{N}$ frames that were transmitted and sends a SREJ for only those frames that were received in error. The source, on receiving the SREJ, retransmits only those frames for which the SREJ was received. Fig. 7.4 indicates a sample timing diagram of selective-repeat ARQ.

In the example shown by Fig. 7.4 , the destination sends a negative acknowledgment for frames 3 and 5 . On receiving the SREJ, the source only retransmits frames 3 and 5 that were in error.

Selective-repeat ARQ seems to be more efficient as compared to go-back-N ARQ because only those frames are retransmitted for which a negative acknowledgment has been received. Although the selective-repeat SRQ is more efficient, it requires large memory buffers for incoming data and complex logic to ensure proper sequencing of data. 


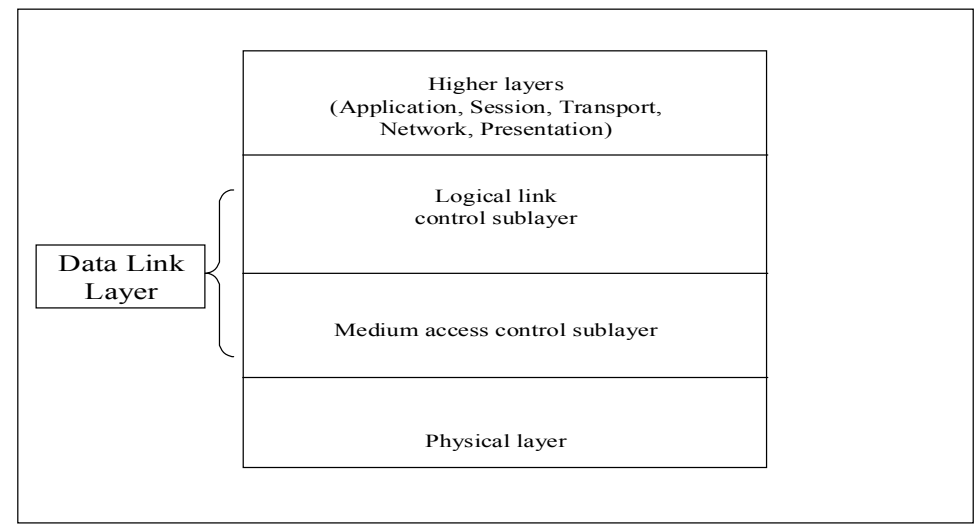

Figure 7.5: OSI reference model

\subsection{Power Line Communication System Design}

Familiarity with the power line channel characteristics and the ability to model the channel can provide for an approach to system implementation for such a link. System design and implementation would include the following:

\subsubsection{Protocol Design}

A three-layered protocol structure comprising the physical layer, medium access control (MAC) sublayer and logical link control (LLC) sublayer, as proposed in [42] can be adopted for such a system. The MAC and LLC sublayers form part of the data link layer in the open system interface (OSI) seven-layer model, as indicated in Fig. 7.5.

The LLC sublayer is primarily responsible for providing the destination service access point (DSAP), source service access point (SSAP) and higher layer data. The LLC sublayer also handles the ARQ protocol. The MAC sublayer is primarily responsible for adding the destination and source physical addresses, flags, priority settings, and error control code (CRC) to the stream of data supplied by the LLC sublayer (LLC-PDU). The MAC sublayer also provides for access to the physical layer by means of an access protocol. The access protocol considered for the PLC system design is loosely based on the CEBus standard adopted by North America as the protocol for home automation communication purpose. 


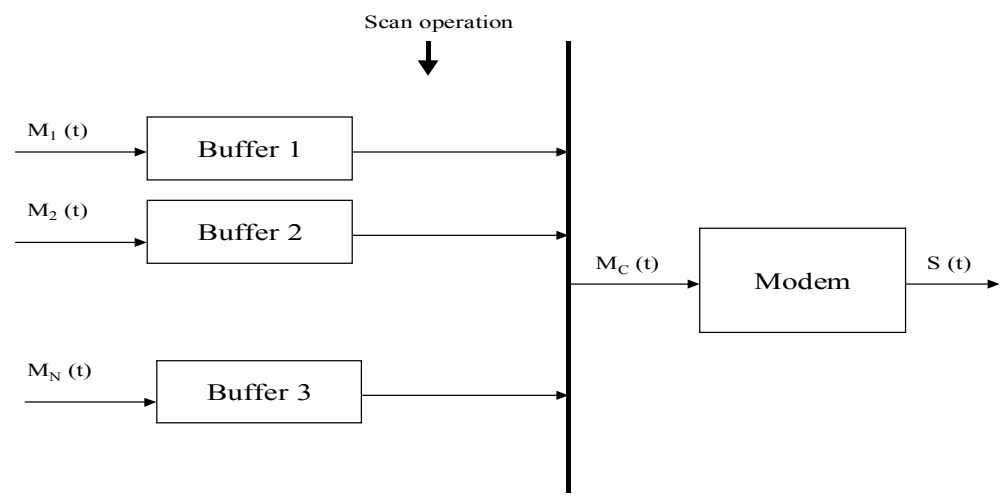

Figure 7.6: Block diagram of TDMA transmitter

\subsubsection{Access Protocol}

The channel can be accessed using schemes such as time division multiple access (TDMA) or carrier sense multiple access (CSMA). In a CSMA system, the transmitter will transmit data only if it senses an idle channel. Once the transmitter senses an idle channel, it might start transmitting immediately or it might wait further for a random amount of time (contention backoff window) before transmitting. In TDMA systems, each transmitter is allocated a certain amount of time in which it can transmit, on a periodic basis. Depending on the priority of the transmitter, more time can be allocated to those transmitters having higher priority. Similar priority settings are also possible in case of CSMA systems by assigning shorter backoff periods to higher priority transmitters. The two access protocols, CSMA and TDMA, are detailed as follows:

- TDMA: In TDMA, the data is organized into frames and each user is allocated time slots for communication purpose. Each frame is sent over multiple time slots [64].

All the users in the system transmit on the same frequency but at different time intervals. This mode of operation is generally called synchronous TDMA since the time slots are fixed and pre-assigned to individual users. Fig. 7.6 provides the block diagram of a TDMA transmission system. Each user is provided a time slot and the data that needs to be transmitted is stored in the buffer. Data from the buffer is 


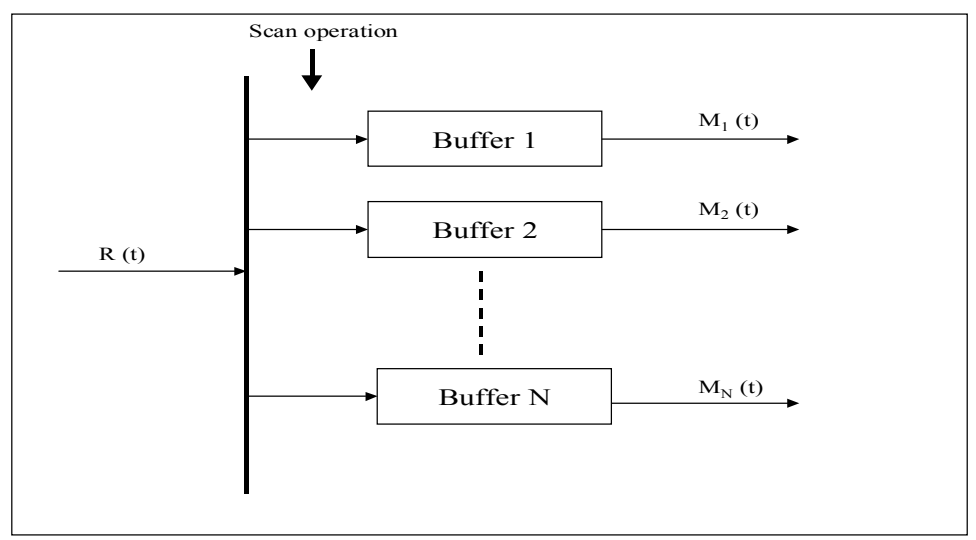

Figure 7.7: Block diagram of TDMA receiver

transmitted at individual time slots by the scanning operation.

Fig. 7.7 indicates a TDMA reception system. The scanning operation is performed according to the time slot allocated for each individual user and the scanned data is passed onto the user buffer. Guard time bands are allocated between user time slots to minimize interference between users. TDMA systems have high overhead and low efficiency because of synchronization requirements and guard bands in between time slots.

- CSMA: In CSMA, a station that needs to transmit first listens to the channel to determine if any transmission is in progress. If the station detects a busy channel, it waits for a predetermined time before listening to the channel again. If the station finds the channel to be idle, it starts transmitting immediately. If two or more stations begin to transmit at the same time, the resulting collision corrupts the data frames transmitted by the individual stations. If such a collision occurs, the channel remains unusable for the entire period of transmission of all the frames, thus affecting channel utilization. However, channel utilization can be improved by using CSMA/CD (collision detection), which is the access protocol for wired LANs, or by using CSMA/CA (collision avoidance), which is the access protocol for radio LANs. In a CSMA/CD 
system, if the source detects a collision ${ }^{1}$, it broadcasts a jamming signal to all the other stations informing them that a collision has occured and ceases to transmit. In a CSMA/CA system, if the source detects an idle channel, it will broadcast a signal to all the other stations informing them that it is about to begin its transmission. CSMA systems are easy to implement and hardware costs are low. Token ring access protocol can also be considered for PLC system design, but they are expensive to implement and serious problems could arise with lost tokens or misinterpretation of tokens on noisy channels.

\subsubsection{A Combined CSMA/ARQ Protocol}

The following algorithm can establish the PLC CSMA/ARQ system [65], as follows:

1. Transmit if the channel is sensed idle.

2. Wait for the Positive acknowledgment (ACK) / Negative acknowledgment (NACK) signal from the receiver, which will indicate that the transmission was successful or not.

3. If a NACK is received and if the channel is sensed idle, then retransmit after waiting for a random period of time (exponential backoff).

4. If an ACK is received, then transmit the next packet in sequence as soon as the channel is sensed idle.

This kind of a stop-and-wait ARQ/CSMA scheme ensures that the transmission will take place only on reception of the ACK or the NACK signal. Though this system might be a little slower than some other ARQ systems described previously, it will ensure reliable communications through a hostile and noisy channel such as the power line channel.

\footnotetext{
${ }^{1}$ Collision detection by the source occurs when the source detects a signal whose voltage exceeds the maximum signal voltage that the source can produce alone.
} 


\begin{tabular}{|l|l|l|l|l|l|l|}
\hline Flags & $\begin{array}{c}\text { Destination } \\
\text { Address }\end{array}$ & $\begin{array}{c}\text { Source } \\
\text { Address }\end{array}$ & Control & Coded Data & Flags & CRC \\
\hline
\end{tabular}

Figure 7.8: Packet structure for PLC

\subsubsection{Packet Structure}

The packet structure that can be implemented is loosely based on the CEBus standard adopted in North America. Fig. 7.8 defines the packet structure. The two flag fields are each 1 byte long. The destination and source addresses, and the control field are 4 bytes long. The data payload is of variable length. The CRC uses a 16-bit generator polynomial given by $X^{16}+X^{15}+X^{2}+1$, which is known as the CRC-16 version ${ }^{2}$.

The flags can be used to indicate whether the packet is the first packet or the last packet or part of a bigger packet that has been fragmented. The flags can also be used to indicate the start or the end of a sequence of data and also whether the data is fixed-length or variable-length. The destination and source address indicate the respective nodes that will act as the transmitter and the receiver. It can also be used to define the transaction of data between the central server and the nodes. The control fields can be used for channel contention control.

The data can either be FEC-encoded or uncoded. Coding the data will help in improving the BER performance of the communication system implemented. Convolutional or Reed-Solomon coding could be used at the transmitter and the Viterbi decoder or the Berlekamp-Massey algorithm can be used, respectively, at the receiver to decode the data. The control part of the packet will be responsible for controlling the flow of data. It can also be used to specify the length of the packet and could also be useful for adding security

\footnotetext{
${ }^{2}$ Intellon uses the CRC-32 version for its power packet structure.
} 
options.

\subsection{Throughput Considerations}

Throughput is defined as the number of bits received correctly to the total number of bits that were transmitted by the receiver. It is very important to understand the throughput efficiency of the stop-and-wait ARQ protocol, for the PLC system, in order to understand the channel efficiency in the presence of bit errors. For the stop-and-wait ARQ protocol, assuming error-free transmissions, the throughput efficiency $\eta$ is defined as

$$
\eta=\frac{t_{\text {frame }}}{2 t_{\text {prop }}+t_{\text {frame }}}
$$

where $t_{\text {frame }}$ is the time required to transmit a single frame and $t_{\text {prop }}$ is the propagation time from the source to the destination. However, in practical PLC systems, there will be packets in error. Hence, if $\bar{N}_{r}$ is the expected number of transmissions of a single frame before it is received correctly, then the throughput efficiency can be modified as

$$
\eta=\frac{1}{\bar{N}_{r}(1+2 a)}
$$

where the parameter $a$ can is defined such that

$$
\begin{aligned}
a & =\frac{t_{\text {prop }}}{t_{\text {frame }}} \\
& =\frac{R d_{i}}{v L}
\end{aligned}
$$

where $R$ is the bit rate of the communication system, in bps, $d_{i}$ is the distance between the source and the destination, in meters, $v$ is the velocity of light in the medium, in meters per second, and $L$ is the total number of bits in the frame. The value of $a$ for the example PLC system can be calculated by assuming the following parameters: The bit rate $R$ is 1 Mbps, the distance between the source and destination $d_{i}$ is 1000 meters, the velocity of light $v$ in the medium is 0.67 times the velocity of light in free space, and according to the simulations performed for the PLC system, there are 1024 bits in each frame. Hence the value of $a$ can then be easily calculated for the PLC system as 0.0049 . 
If $N_{r}$ is the number of transmissions, and $\bar{N}_{r}$ is the expected number of transmissions of a single frame, then

$$
\begin{aligned}
\bar{N}_{r} & =E\left[N_{r}\right] \\
& =\sum_{m=1}^{\infty} m P_{N_{r}}[m] \\
& =\sum_{m=1}^{\infty} m P^{m-1}(1-P) \\
& =\frac{1}{1-P}
\end{aligned}
$$

where $P$ is the probability that the frame is in error ${ }^{3}$. Hence the throughput efficiency $\eta$ can be written as

$$
\eta=\frac{1-P}{1+2 a}
$$

Since, there are 1024 bits in each frame,

$$
P=1-\left(1-P_{b}\right)^{1024}
$$

where $P_{b}$ is the bit error probability.

Hence, in terms of the bit error probability $P_{b}$, the throughput efficiency $\eta$ can be written as

$$
\eta=\frac{\left(1-P_{b}\right)^{1024}}{1+2 a}
$$

The value of $a$, as previously obtained, is 0.0049. Using Eqn. (7.11), a plot of throughput efficiency for different values of bit error probabilities can be obtained, which is given by 7.9. The plot indicates that the throughput efficiency of a CSMA system increases with decreasing BER and that a BER of at least $10^{-3}$ should be targetted to obtain acceptable performance (a throughput efficiency of around 0.5) in a PLC system implementation. The plot also acts as a useful tool in PLC system design. For a desired throughput efficiency, the corresponding BER can be obtained from the plot and the BER performance curves from the previous chapters could be used to obtain corresponding signal-to-noise ratios at the receiver.

\footnotetext{
${ }^{3}$ The relationship $\sum_{m=1}^{\infty} m P^{m-1}=\frac{1}{(1-P)^{2}}$ was used
} 


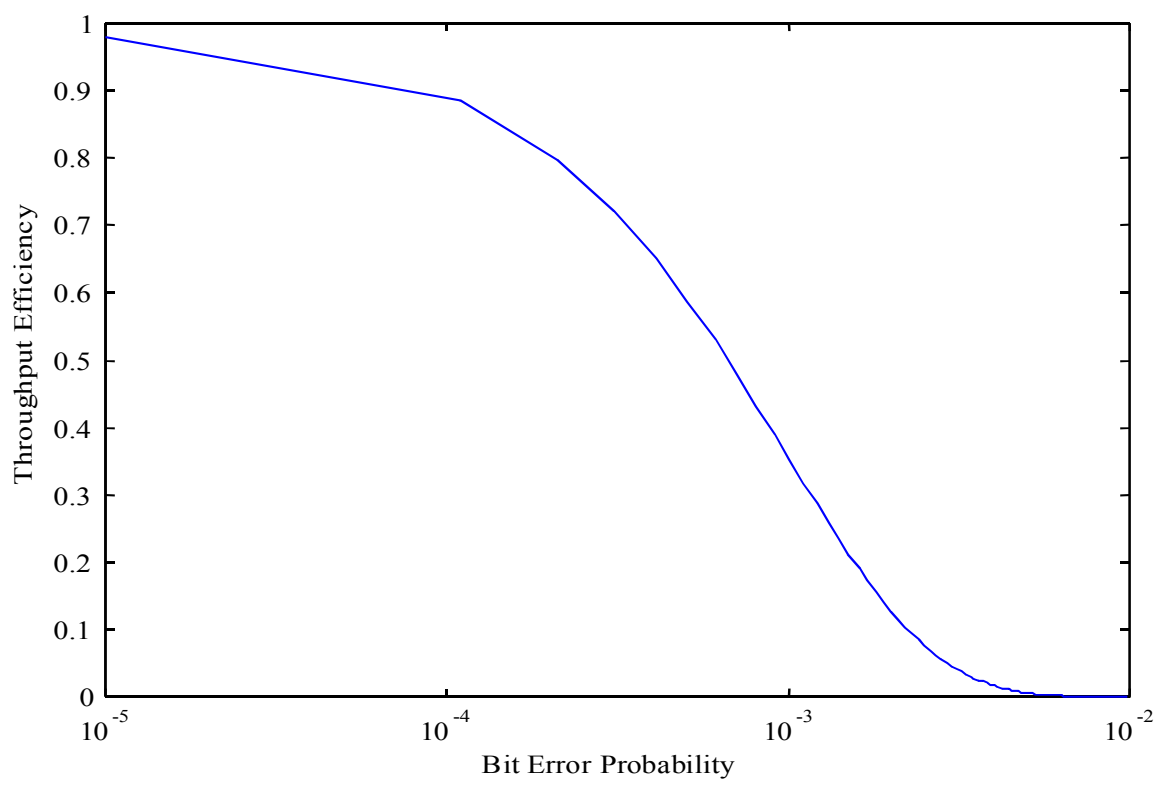

Figure 7.9: Throughput versus BER for the combined CSMA/ARQ protocol and for a packet size of 1024 bits

\subsection{Conclusion}

This chapter provided an insight into the actual implementation procedure that needs to be performed and a discussion of issues related to layers above the physical layer of a system. Specific issues like error control and medium access were discussed in detail. An example protocol was specified, and its throughput computed. 


\section{Chapter 8}

\section{PLC System Implementation and Simulations}

\subsection{Introduction}

Power line communications (PLC) is fast emerging as an alternative communication medium. Utilities can use PLC not only for the purpose of monitoring and tracking the power grid but also to provide broadband access to their customers. Many organizations and utilities around the world are actively involved in the research and development of equipment that can be used for power line communications. Power line channel models are used to a great extent in studying the characteristics of the power line medium. This chapter looks at how the digital communication techniques like spread spectrum (SS) and orthogonal frequency division multiplexing (OFDM), described in the previous chapters, can be effectively used to combat a hostile communication medium like a power line. The chapter concludes by simulating the various techniques with the help of the suggested power line channel model.

\subsection{History of PLC}

Power line communications dates back to the 1920's when the process of electrification had just started [33]. Utilities at that time primarily depended on power lines for data transmission because the telephone network was very unreliable at that time. PLC was also used in those days for remote monitoring due to the unavailability of telephone 
networks at remote locations. In those days, continuous wave modulation like linear modulation and frequency modulation were used as the primary modulation techniques.

In linear modulation, the message signal $m(t)$ is multiplied by the carrier signal $A_{c} \cos \left(\omega_{c} t\right)$ such that the amplitude of the carrier signal varies linearly with the amplitude of the message signal [64]. The output of the modulator $x(t)$ can be expressed as

$$
x(t)=A_{c} m(t) \cos \left(\omega_{c} t\right)
$$

The Fourier transform of the above equation leads to

$$
X(f)=\frac{1}{2} A_{c} M\left(f-f_{c}\right)+\frac{1}{2} A_{c} M\left(f+f_{c}\right)
$$

where $X(f)$ is the Fourier transform of $x(t)$ and $M(f)$ is the Fourier transform of $m(t)$. Eqn. (8.2) indicates that there are two signal components, one at $\left(f-f_{c}\right)$, and the other signal component at $\left(f+f_{c}\right)$,. The spectra of the 2 components do not overlap each other since the carrier frequency $f_{c}$ is much larger than the bandwidth of the message signal $W$. A Costas phase-locked loop can be used at the receiver end to coherently demodulate the transmitted signal.

Sometimes, in order to reduce the bandwidth from $2 W$ to $W$, only one of the sidebands (message sideband before modulation) is transmitted. This method is known as single side band (SSB) transmission. Filters with sharp cut-off have to be used in order to obtain the tranmitted signal. Vestigial sideband (VSB) transmission reduces the complexity of single side band due to the relaxation of the cut-off slope of the filters at both the transmitter and the receiver.

Amplitude modulation (AM), also called double sideband large carrier (DSB-LC), is a form of linear modulation wherein a DC bias $A$ is added to the message signal $m(t)$, such that the output of the modulator is given as

$$
x(t)=[A+m(t)] A_{c} \cos \left(\omega_{c} t\right)
$$

The advantage of providing a DC bias is the fact that simple envelope detectors can be used at the demodulator to recover the transmitted signal. The modulation index $\mu$ of an 
AM signal is defined as

$$
\mu=\frac{|\min (m(t))|}{A}
$$

Envelope detectors can be used to detect the signal only if $\mu \leq 1$.

In frequency modulation (FM) systems, the frequency deviation of the carrier signal is proportional to the message signal such that

$$
\frac{d \phi(t)}{d t}=K_{f} m(t)
$$

where $\phi(t)$ is the phase of the carrier signal and

$$
K_{f}=2 \pi f_{d}
$$

where $f_{d}$ is the frequency deviation constant. Hence the signal at the output of the frequency modulator output is given by

$$
x(t)=A_{c} \cos \left[\omega_{c} t+2 \pi f_{d} \int_{t_{0}}^{t} m(\alpha) d \alpha\right]
$$

A differentiator coupled with a bandpass filter and an envelope detector can be used to demodulate FM signals.

Apart from the use of AM and FM, ripple carrier signaling (RCS) was also used to communicate telemetric data over medium and low voltage power lines [33]. RCS systems operated at a carrier frequency very close to the power signal frequency and had extremely low data rates (on the order of a few bits per second) in order to maintain reliable communications in the presence of strong interference from the power signal. The main advantage of RCS signaling was the fact that since it was operating at low frequencies, the data signal could easily pass through transformers with very little attenuation and hence RCS systems were primarily used on data networks with a large number of transformers inherent to the system [33].

Presently, digital communication techniques like SS and OFDM are being used to mitigate the hostile effects of the power line channel and provide data rates on the order of few Mbps. These systems operate at frequencies far way from the power signal frequency and thus can operate at low power, as compared to RCS systems. 


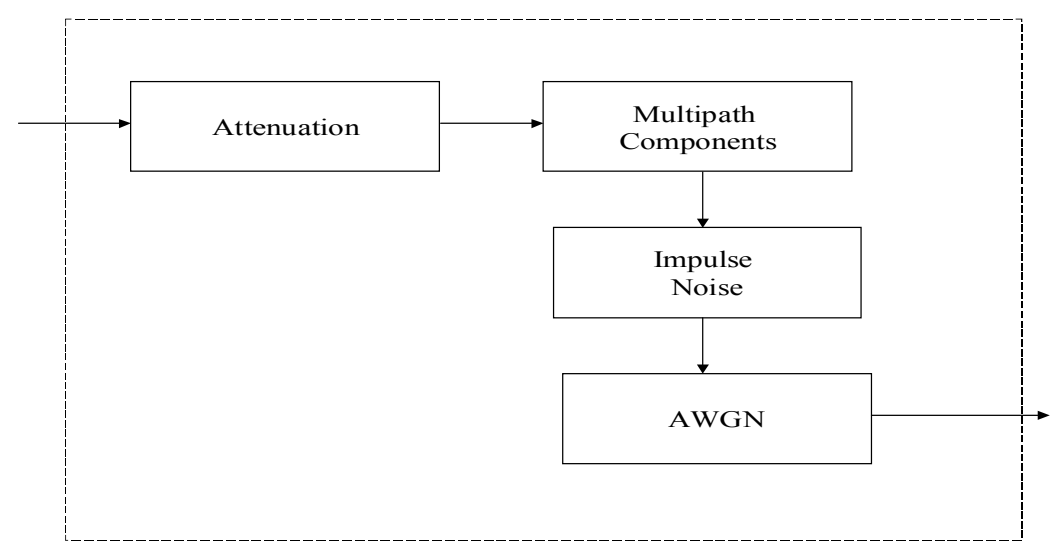

Figure 8.1: Power Line Channel Model

\subsection{The Power Line Channel}

A complete model for a power line channel would include features like signal attenuation, background noise that can be modeled as additive white Gaussian noise (AWGN), additive impulse noise, fading and multipath components of the signal. As stated earlier, signal attenuation is dependent on the physical length of the power line cable over which the information data has to be transported and on the frequency of the signal. The background noise, modeled as AWGN, is primarily because of interference from sources such as radio \& TV broadcasts, cosmic radiation, and thermal noise in the receiver circuitry. Noise in the form of impulsive and non-impulsive noise is an important factor, which should be included in the modeling of residential power line channel. Impulsive and non-impulsive noise occurs due to the presence of switching power systems in electronic equipments and due to the use of universal motors in residences respectively.

The impulse response of the power line channel $h(t)$ can be summarized as

$$
h(t)=A\left(d, f_{o}\right) \sum_{i=1}^{N} c_{i} \delta\left(t-\tau_{i}\right)
$$

where $N$ is the number of multipath components, $c_{i}$ is the fading channel coefficient associated with the $i^{t h}$ multipath component, $A$ is the signal attenuation of the signal which is dependent on the frequency of operation $f_{o}$ and the transmitter-receiver seperation $d[36]$. 


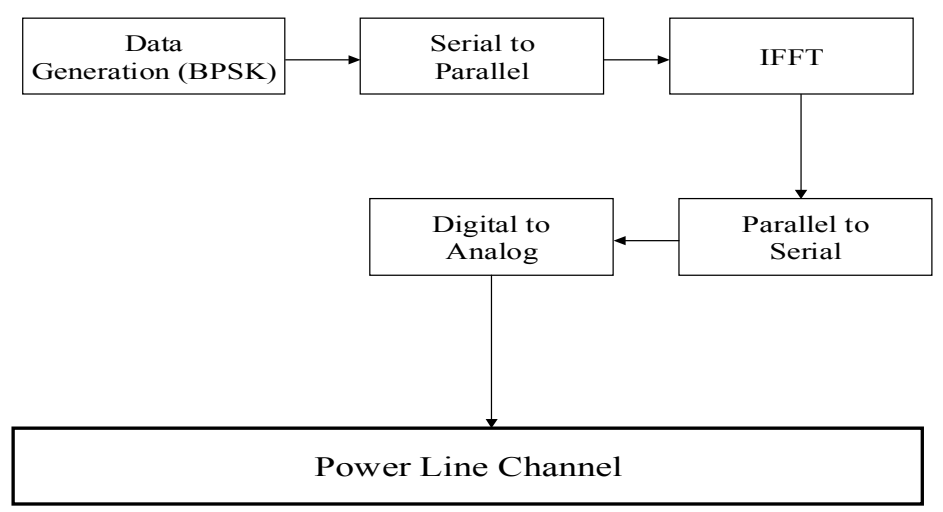

Figure 8.2: Basic OFDM transmitter

\subsection{PLC Simulations}

Digital modulation techniques like SS and OFDM are used in real-world PLC applications. Spread spectrum techniques provide the data signal with immunity from narrowband interference like harmonic and non harmonic impulse noise. OFDM, on the other hand, is a useful technique for mitigating the effects of a frequency-selective fading channel like the PLC channel. OFDM converts the high data rate signal into several parallel low data rate signal channels and transmits all the channels, on orthogonal frequencies, at the same time. This results in the conversion of a single frequency selective channel into multiple flat fading channels, which is useful for combating multipath propagation, since the symbol period will be greater than the channel coherence time. Fig. 8.2 represents the block diagram of an OFDM system and Fig. 8.3 represents the block diagram of an OFDM receiver.

Simulations were performed to test the performance of SS and OFDM. Both modulation techniques used BPSK signaling. For the SS simulation, a random PN code was generated for each frame of data, each frame containing 1024 bits of data. The impulse response of the PLC channel model used, is given by Eqn. (8.8). For the SS simulation, a processing gain of seven was used and for the OFDM simulation, a total of eight subcarriers were considered. The delays $\tau_{i}$ for the multipath components were assumed to be random but within the bit period $T_{b}$, and integral multiples of the chip period $T_{c}$. The weighting coefficients $c_{i}$ were assumed to be real and they were assumed to be 1 and 0.64 for the 


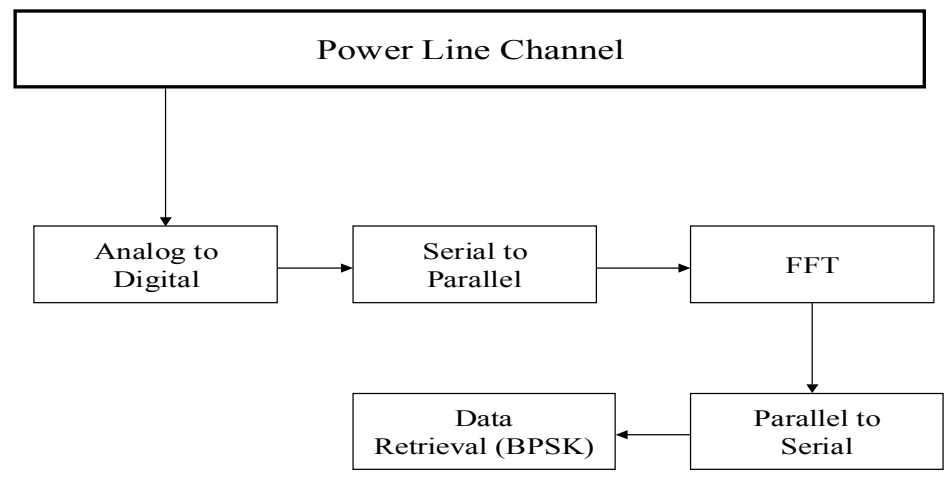

Figure 8.3: Basic OFDM receiver

respective multipath signal components. The value of 0.64 was obtained from [36], which contained various experimental values for the weighting coefficients. The multipath signals were normalized for the simulation since the signal to noise ratio (SNR) was defined using the average signal power at the receiver.

It was also assumed that only one user is able to access the channel at any given point of time, which is true in the case of network access protocols like CSMA or TDMA. Hence, apart from noise sources, only the multipath components of the desired user was considered as interference. Fig. 8.4 indicates the performance of SS and OFDM, uncoded, in a PLC channel. A rake receiver with two fingers was used to capture signal information from the multipath components, with the assumptions that the fingers are perfectly tuned to the delay of the signal multipath components and that only two multipath signal components are enough to extract most of the signal information. The graph clearly indicates that a rake receiver can help improve BER performances in the presence of multipath propagation. The BER graph also indicates that OFDM performs better than SS in a PLC channel, at least in this case.

\subsection{Comparison of DS-SS and OFDM}

In an OFDM system, since there are a large number of subcarriers, the combined signal has a very large peak-to-average power ratio (PAPR), and to maintain linearity over 


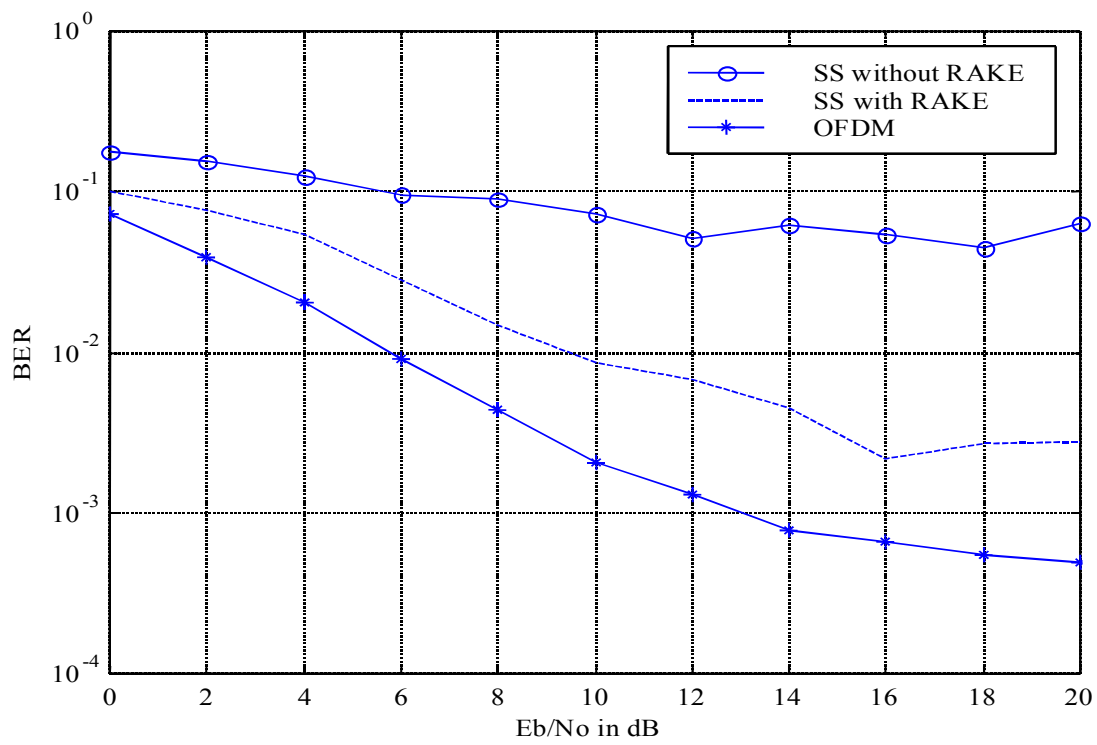

Figure 8.4: BER performance of uncoded SS without rake receiver, uncoded SS with 2-finger rake receiver, and uncoded OFDM in a PLC channel

the frequency range, the power amplifiers used have to be very dynamic in the particular frequency range. Because it is a single carrier system, DS-SS can use constant envelope modulation and hence does not have this problem.

OFDM systems allow for dynamic and discontiguous frequency allocation of the different subcarriers in an effort to mitigate the effects of a frequency selective channel or signal around interfering sources. In fact, this is an important concept being effectively used by chip makers like Intellon and Inari to combat the hostile nature of the power line channel. SS systems are much more limited in terms of dynamic frequency allocation due to its single carrier nature.

SS systems do not require the overhead of guard bands and cyclic prefixes as in the case of OFDM (which are used to avoid inter symbol interference (ISI)). ISI occurs due to the loss of orthogonality among the subcarriers while being transmitted through the channel. This overhead tend to reduce data throughput. However, practical OFDM applications have data rates around 10-15 Mbps and practical SS systems have data rates 
around $5 \mathrm{Mbps}$. This indicates that in spite of the overhead, OFDM provides for higher data transmission rates.

Frequency offsets between the transmitter and the receiver play an important role especially in the case of OFDM systems. Frequency offsets must be removed with automatic frequency control (AFC) or else the subcarriers will not continue to be orthogonal to each other. SS systems need to take care of timing offsets between the transmitter and the receiver, and this becomes increasingly crucial with increasing processing gains. The chip maker Intellon uses a processing gain of 2520 for their SS systems (2520 chips for every bit of data).

Multipath propagation is an important aspect of power line communications since it is a frequency selective channel by nature. OFDM systems have an advantage here because the OFDM symbols see a flat fading channel instead of a frequency selective channel, thereby mitigating the interference effects of multipath propagation. SS systems, on the other hand, suffer from multipath signal interference because of the frequency selective nature of the channel. Rake receivers could be used in conjunction with SS systems in order to extract information from the multipath signal components. However, the use of rake receivers increases the complexity of the SS system.

\subsection{Possible Applications of PLC}

Possible PLC applications can be divided into the following categories:

- Monitoring and control: This is the most important aspect of utility communications. Technical parameters are fed from different measuring instruments, located at remote terminal units (RTUs) and system protection terminals (SPT) into the central processing unit in order to monitor and control the grid. In wide area measurement systems (WAMS), measuring instruments like analog transducers and PMUs are strategically placed throughout a wide coverage area and these form part of the system protection terminals [7]. 
SPTs are able to run complete or parts of distributed control algorithms and can communicate directly with other SPTs, substation equipment and system protection centers (SPC), which are responsible for monitoring and control of the power grid.

- Data exchange: Wide area protection is not possible if utilities are not able to exchange information with each other. Exchange of parameters and data between utilities is important as local outages in the control area of one utility could cascade into a local outage in the control area of the other utility. The data exchange working group of the North American Electricity Reliability Council (NERC) decided to adopt the common information model (CIM) as the common protocol which would enable utilities to communicate with one another [66].

The CIM is based on the IEEE self defining protocol (SDP) and basically consists of source information, destination information, and the data concerned. The CIM is programmed using eXtensible markup language (XML) which can be run using standard web browser technology. NERC decided to go with XML as the language for CIM primarily because XML can be accessed using any standard web browser and is much more flexible as compared to HTML, in terms of determining the data type definitions (DTD). Apart from dynamic DTD, XML also is easy to program and understand. Fig. 8.5 represents a simple XML program to indicate the ease and flexibility of programming with XML.

- Substation communication: Apart from the above mentioned types of utility communications, substation communications also play an important role as it is the substations that decide on the actual working of instruments that control the grid, based on the parameters received from the various measuring instruments. In 1990, the electric power research institute (EPRI) decided on a protocol known as the utilities communication architecture (UCA) as the platform for intra-substation communication [67].

Fig. 8.6 represents the architecture of the UCA protocol [68]. TCP/IP was adopted 


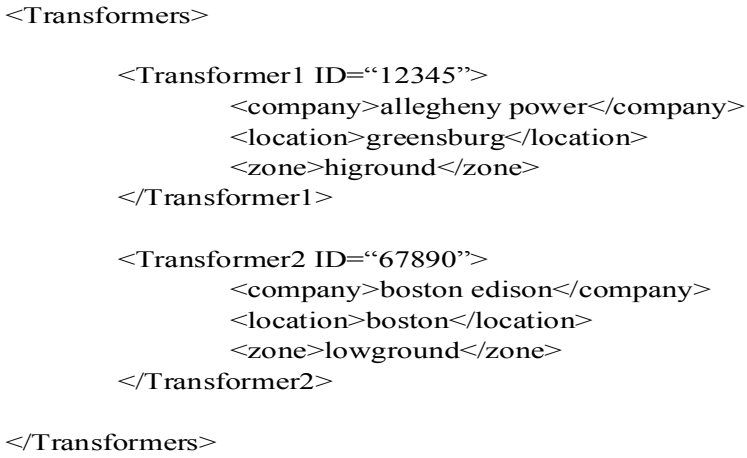

Figure 8.5: Sample XML program

as the network layer because of its common usage. Manufacturing messaging specification (MMS) provides services such as read, write, get file, get list of file, etc. to the relays and supports object oriented data definitions as well. Common Application Service Models (CASM) provides the mapping of the substation specific applications such as Select Before Operate, Exception Reporting, and File operations into the underlying MMS services. Generic Object Models for Substation \& Feeder equipment (GOMSFE) defines standard data models for equipment and functions found in the substation.

\subsection{PLC Case Studies}

Organizations are increasingly understanding the importance of PLC and some of the case studies reviewed are as follows:

1. Intellon is one of the leading companies involved in PLC and manufactures PLC products in collaboration with electronic giants like Motorola, IBM, Nortel, and Intel to name a few, as part of a coalition towards developing home energy management systems. Intellon power packet technology based on spread spectrum carrier has a data rate around $5 \mathrm{Mbps}$ and OFDM based technique has a data rate around $12 \mathrm{Mbps}$. 


\begin{tabular}{|c|}
\hline $\begin{array}{c}\text { Generic Object Models for Substation and } \\
\text { Feeder Equipment (GOMSFE) }\end{array}$ \\
\hline $\begin{array}{c}\text { Common Application Service } \\
\text { Models (CASM) }\end{array}$ \\
\hline $\begin{array}{c}\text { Manufacturing Messaging } \\
\text { Specification (MMS) }\end{array}$ \\
TCP / IP \\
Physical Layer
\end{tabular}

Figure 8.6: UCA architecture

2. Ambient Corporation and Southern Telecom, a subsidiary of Southern Company, have jointly entered into a comprehensive testing and development agreement which provides for joint development and testing of first generation products that will lead to the deployment of Ambients proprietary Power Line Communications (PLC) technology over Southern Telecoms affiliates test facilities and electrical power grids. The testing will include overhead and underground systems, as well as medium and low voltage levels ${ }^{1}$.

3. Ambient Corporation and Consolidated Edison Company of New York, one of the nations largest investor-owned utilities, jointly announced the conclusion of a comprehensive Research and Development so that Ambient can immediately begin to test and deploy its proprietary PLC technology over Consolidated Edisons electrical power lines and grid.

4. Electric grid control authorities in Guam (Guam Power Authority (GPA)), which comes under NERC - region nine, has installed a PLC communication system as a backup to its fiber-optic network system.

\footnotetext{
${ }^{1}$ www.ambient.com
} 
5. Amperion, Inc., is a premiere medium voltage powerline networking equipment company. Amperion develops networking hardware and software that enables the delivery of high-speed broadband data over the medium-voltage power line (MV-PLC) assets of the Utilities. Amperion's products will enable customers to use the existing power line infrastructures to provide high-speed data transport, which will enable Broadband service providers of any type to expand their offering and reach new markets in a competitive environment. First round investors in Amperion include, American Electric Power, one of America's largest distributors of electricity, Cisco Systems, Inc., the worldwide leader in networking for the Internet, and Redleaf Group, Inc., a leading technology operating company ${ }^{2}$.

6. Ameren has partnered with Main.net Communications to develop, market and sell a complete broadband communication solutions over power lines. Ameren has also partnered with Big River Telephone Company in Cape Girardeau, MO. Big River Telephone Company offers local, long distance and Internet connections to customers primarily in southeastern Missouri, southern Illinois and western Kentucky.

7. Potomac Electric and Power Company (PEPCO) has announced trial runs of power line communications with Current Technologies, a Maryland based company.

\subsection{Conclusion}

This chapter presented a detailed look at the characteristics of the power line channel. Simulations were performed which indicated the performance of spread spectrum and OFDM systems. The advantages of using a rake receiver were also observed. In the earlier days, PLC was used only as a communication medium for transfer of parametric data, at very low data rates and at high power. Present day modulation techniques like SS and OFDM are converting the extremely hostile power line channel into an optimum medium for both wide area measurement systems and broadband communications.

\footnotetext{
${ }^{2}$ www.amperion.com
} 


\section{Appendix A}

\section{List of Variables}

$N_{o}$ Two-sided noise spectral density.

$n$ Additive white Gaussian noise.

$N_{j}$ One-sided average jammer power spectral density.

$w_{1}, w_{2}$ Weighting coefficients of the rake receiver fingers.

$G$ Processing gain

$W$ Signal bandwidth

$b$ Message sequence of the user

$E_{b}$ Energy per bit

$s_{j}(t) \mathrm{PN}$ code sequence of user $\mathrm{j}$

$s_{k}(t)$ PN code sequence of user $\mathrm{k}$

$\tau$ Delay between bit epochs for asynchronous transmission

$\eta$ Throughput efficiency (channel utilization)

$P_{b}$ Bit error probability

$P$ Frame or Packet error probability

$N_{r}$ Number of transmissions of a single frame 
$\bar{N}_{r}$ Expected number of transmissions of a single frame

$T_{b}$ Bit period of the message signal

$T_{c}$ Chip period of the $\mathrm{PN}$ code sequence

$\rho$ Correlation coefficient

$y$ Output of the matched filter

$\alpha$ Channel coefficients

$K$ Number of users in the CDMA system

$K_{c}$ Constraint length of a convolutional code

$r$ Code rate

$k$ Number of input bits to the encoder

$u_{n}$ Output after IFFT operation on a block of data

$R$ Bit rate in bps

$d_{i}$ Physical distance between the transmitter and the receiver

$V$ Velocity of light in the particular communication medium

$L$ Number of bits per packet or frame

$a$ Ratio of propagation time to transmission time

$m$ Number of transmissions of a single frame

$A_{c}$ Amplitude of modulating carrier signal

$m(t)$ Message signal

$M(f)$ Fourier transform of message signal $m(t)$

$x(t)$ Modulated signal

$X(f)$ Fourier transform of modulated signal $x(t)$ 
$f_{c}$ Carrier frequency

$f_{d}$ Frequency deviation constant

$c_{i}$ Weighting coefficient of the $i^{t h}$ multipath component

$d_{i}$ Signal path length

$f_{o}$ Frequency of operation of the PLC system 


\section{Appendix B}

\section{List of Acronyms}

PLC Power Line Communications

SS Spread Spectrum

OFDM Orthogonal Frequency Division Multiplexing

PMU Phasor Measurement Unit

WAMS Wide Area Measurement Systems

WAP Wide Area Protection

ISO Independent System Operator

PX Power Exchange

CDMA Code Division Multiple Access

FDMA Frequency Division Multiple Access

TDMA Time Division Multiple Access

CSMA Carrier Sense Multiple Access

ARQ Automatic Repeat Request

SPC System Protection Center

SPT System Protection Terminal 
SCE Southern California Edison

DR Distributed Resources

UCA Utilities Communication Architecture

XML Extensible Markup Language

EPRI Electric Power Research Institute

PV Photo Voltaic Cells

FACTS Flexible AC Transmission Systems

AMR Automated Meter Reading

SCADA Supervisory Control and Data Acquisition

IED Intelligent Electronic Device

EMS Energy Management Systems

VSAT Very Small Aperture Terminal

BER Bit Error Rate

ROI Return on Investment

AWGN Additive White Gaussian Noise

OSI Open Systems Interconnect

DFT Discrete Fourier Transform

FFT Fast Fourier Transform

IFFT Inverse Fast Fourier Transform

GPS Global Positioning Satellite

SA Selective Availability

AM Amplitude Modulation 
FM Frequency Modulation

UPFC Universal Power Flow Controllers

VSC Variable Series Capacitor

BCD Binary Coded Decimal

UTC Universal Time Co-ordinates

CRC Cyclic Redundancy Check

SOC Second of Century

SMPCNT Sample Count

LED Light Emitting Diode

LASER Light Amplification by Stimulated Emission of Radiation

WDM Wavelength Division Multiplexing

DTE Data Terminal Equipment

DCE Data Communication Equipment

BPSK Binary Phase Shift Keying

QAM Quadrature Amplitude Modulation

SNR Signal to Noise Ratio

ISI Inter Symbol Interference

DSSS Direct Sequence Spread Spectrum

FHSS Frequency Hopping Spread Spectrum

ADC Analog to Digital Converter

RTU Remote Terminal Unit

DLL Data Link Layer 
MAC Medium Access Control

LLC Logical Link Control

CIM Common Information Model

SDP Self Defining Protocol

NERC North American Electricity Reliability Council 


\section{References}

[1] B. Barker, "The electricity technology roadmap," EPRI Journal, pp. 25-31, November/December 1998.

[2] T. Moore, "Emerging markets for distributed resources," EPRI Journal, pp. 9-17, March/April 1998.

[3] F. Albuyeh and Z. Alaywan, "California ISO formation and implementation," IEEE Computer Applications in Power, pp. 30-34, October 1999.

[4] A. Cohn, R. Goldstein, and D. Rastler, "Market potential for fuel cells," EPRI Journal, 1996.

[5] J. Douglas, "Power delivery in the 21st century," EPRI Journal, Summer 1999.

[6] L. Lamarre, "At home with communications," EPRI Journal, pp. 7-15, January/February 1997.

[7] C. Rehtanz and J. Bertsch, "A new wide area protection system," in 2001 IEEE Porto Power Tech Conference, vol. 4, (Porto, Portugal), pp. 186-191, September 2001.

[8] A. M. Wildberger, "Complex adaptive systems : concepts and power industry applications," IEEE Control Systems, pp. 77-88, December 1997.

[9] A. G. Phadke, "Synchronized phasor measurements in power systems," IEEE Computer Applications in Power, vol. 6, pp. 10-15, April 1993.

[10] IEEE Standards Board, IEEE Standard for Synchrophasors for Power Systems, December 1995. IEEE Std. 1344-1995.

[11] K. Cho, J. Shin, and S. Hyun, "Optimal placement of phasor measurement units with GPS receivers," in Proceedings of the Power Engineering Society Winter Meeting (IEEE, ed.), vol. 1, pp. 258-262, 2001.

[12] B. Bhargava, "Synchronized phasor measurement system project at Southern California Edison Co.," tech. rep., Southern California Edison Co., 1999.

[13] D. G. Hart, D. Novosel, M. Subramanian, and M. Ingram, "Real-time wide area measurements for adaptive protection and control," in NSF/DOE/EPRI Workshop on 
Future Research Directions for Complex Interactive Electric Networks, (Washington, D.C.), November 2000.

[14] V. Centeno, A. Phadke, A. Edris, J. Benton, M. Gaudi, and G. Michael, "An adaptive out-of-step relay," IEEE Transactions on Power Delivery, vol. 12, pp. 61-67, January 1997.

[15] A. G. Phadke, "Computer relaying - its impact on improved control and operation in power systems," IEEE Computer Applications in Power, vol. 1, pp. 5-10, October 1988.

[16] G. P. Agrawal, Fiber-Optic Communication Systems. John Wiley and Sons, Inc., 1992.

[17] J. C. Palais, Fiber Optics Communications. Prentice-Hall, Inc., 1984.

[18] J. Gowar, Optical Communication Systems. Prentice-Hall, Inc., 1984.

[19] S. V. Kartalopoulos, Introduction to DWDM Technology. IEEE Press Marketing, 2000.

[20] G. Keiser, Optical Fiber Communications. McGraw-Hill Book Company, 1984.

[21] Radio Subcommittee of IEEE Power System Communications Committee, "Ana$\log$ /Digital microwave considerations for electric/gas utilities," IEEE Transactions on Power Delivery, vol. 8, pp. 798-815, July 1993.

[22] D. Martin, Communication Satellites 1958 - 1995. The Aerospace Press, 1996.

[23] V. K. Bhargava, Digital Communications by Satellite. John Wiley and sons, Inc., 1981.

[24] L. Schiff and A. Chockalingam, "Signal design and operation of Globalstar versus IS-95 CDMA," Wireless Networks, vol. 6, February 2000.

[25] B. Miller, "Satellites free the mobile phone," IEEE Spectrum, vol. 35, pp. 26-35, March 1998.

[26] T. Moore, "Utility communications go into orbit," EPRI Journal, 1999.

[27] W. Tomasi, Electronic Communications Systems. Prentice-Hall, Inc., 2001.

[28] D. Radford, "Spread spectrum data leap through AC wiring," IEEE Spectrum, pp. 4853, November 1996.

[29] K. M. Dostert, "Frequency hopping spread spectrum modulation for digital communications over power lines," IEEE Journal in selected areas in Communications, vol. 8, pp. 700-710, May 1990.

[30] A. G. Phadke, J. S. Thorp, and K. J. Karimi, "State estimation with phasor measurements," IEEE Transactions on Power Systems, vol. 1, 1986.

[31] J. K. Yook, D. M. Tilbury, and N. R. Soparkar, "Design methodology for distributed control systems to optimize performance in the presence of time delays," in Proc. of the American Control Conference, (Chicago, Illinois), pp. 1959-1964, June 2000. 
[32] N. Biju, M. Valenti, and A. Feliachi, "Communication delays in wide area measurement systems," in Proceedings of SSST-02, vol. 1, pp. 1082-1086, IEEE, March 2002.

[33] K. Dostert, Power Line Communications. Prentice Hall, Inc., 1 ed., 2001.

[34] J. Wang and H. J. Trussell, "Adaptive harmonic noise cancellation with an application to distribution power line communications," IEEE Transactions on Communications, vol. 36, pp. 875-884, July 1988.

[35] M. Tanaka, "High frequency noise power spectrum, impedance and transmission loss of power line in japan on intrabuilding power line communications," IEEE Transactions on Consumer Electronics, vol. 34, pp. 321-326, May 1988.

[36] M. Zimmermann and K. Dostert, "A multipath model for the powerline channel," IEEE Transactions on Communications, vol. 50, pp. 553-559, April 2002.

[37] J. B. O'Neal, "The residential power circuit as a communication medium," IEEE Transactions on Consumer Electronics, vol. CE-32, pp. 567-577, August 1986.

[38] W. Liu, H. P. Widmer, J. Aldis, and T. Kaltenschnee, "Nature of power line medium and design aspects for broadband plc," tech. rep., Ascom Systec AG, 2000.

[39] M. H. L. Chan and R. W. Donaldson, "Amplitude, width and interval distributions for noise impulses on intrabuilding power line communication networks," IEEE Transactions on electromagnetic compatibility, vol. 31, pp. 320-323, August 1989.

[40] P. K. V. der Gracht and R. W. Donaldson, "Communication using pseudonoise modulation on electric power distribution circuits," IEEE Transactions on Communications, vol. 33, pp. 964-973, September 1985.

[41] O. G. Hooijen, "On the channel capacity of the residential power circuit used as a digital communications medium," IEEE Communications Letters, vol. 2, pp. 267-268, October 1998.

[42] J. Onunga and R.W.Donaldson, "Performance of CSMA with priority acknowledgements on intrabuilding power line local area networks," tech. rep., University of British Columbia, 1988.

[43] S. Moshavi, "Multi-user detection for DS-CDMA communications," IEEE Communications Magazine, pp. 124-136, October 1996.

[44] K. Wesolowski, Mobile Communication Systems. John Wiley and Sons, Ltd., 2002.

[45] T. S. Rappaport, Wireless Communications - Principles and Applications. PrenticeHall, 1999.

[46] A. Leon-Garcia, Probability and Random Processes for Electrical Engineering. Addison Wesley, 1994. 
[47] A. J. Viterbi, CDMA : Principles of spread spectrum communications. Addison-Wesley, 1999.

[48] S. Verdu, Multiuser Detection. Cambridge University Press, 1998.

[49] M. K. Varanasi and B. Aazhang, "Near-optimum detection in synchronous codedivision multiple-access systems," IEEE Transactions on Communications, vol. 39, pp. 725-736, May 1991.

[50] Z. Xie, R. T. Short, and C. K. Rushforth, "A family of suboptimum detectors for coherent multiuser communications," IEEE Journal on selected areas in communications, vol. 8, pp. 683-690, May 1990.

[51] A. Papoulis, Probability, Random Variables, and Stochastic Processes. McGraw-Hill, 1991.

[52] R. Ziemer, R. Peterson, and D. Borth, Introduction to Spread Spectrum Communications. Prentice-Hall, 1995.

[53] K. Kettunen, "Enhanced maximal ratio combining scheme for RAKE receivers in WCDMA mobile terminals," IEEE Electronic Letters, vol. 37, pp. 522-524, April 2001.

[54] M. . C. Jeruchim, P. Balaban, and K. S. Shanmugan, Simulation of Communication Systems. Plenum Press, 1992.

[55] J. G. Proakis, Digital Communications. New York: McGraw-Hill, 2001.

[56] W. Hachen, P. Loubaton, S. Marcos, and R. Samy, "Multiple access communications over the power line channel : a CDMA approach," tech. rep., France Telecom, 2001.

[57] S. Lin and D. Costello, Error Control Coding - Fundamentals and Applications. Prentice-Hall, 1983.

[58] M. Russell and G. L. Stuber, "Interchannel interference analysis of OFDM in a mobile environment," tech. rep., Georgia Institute of Technology, 1995.

[59] G. L. Stuber, Principles of Mobile Communication. Kluwer Academic Publishers, 1996.

[60] R. V. Nee and R. Prasad, OFDM for Wireless Multimedia Communications. Artech House, 2000.

[61] L. J. Cimini, "Analysis and simulation of a digital mobile channel using orthogonal frequency division multiplexing," IEEE Transactions in Communications, vol. COM33, pp. 665-675, July 1985.

[62] A.S.Tannenbaum, Computer Networks. Englewood Cliffs, N.J.: Prentice-Hall, 1981.

[63] W. Stallings, Data Computer Communications. 1997.

[64] L. Couch, Digital and Analog Communication Systems. Prentice-Hall, 2000. 
[65] J. O. Onunga and R. W. Donaldson, "Personal computer communications on intrabuilding power line LANs using CSMA with priority acknowledgements," IEEE Journal on selected areas in communications, vol. 7, pp. 180-191, February 1989.

[66] A. deVos, S. Widergren, and J. Zhu, "XML for CIM model exchange," tech. rep., GE-ALSTOM, 2002.

[67] M. Adamiak and W. Premerlani, "Data communications in a deregulated environment," IEEE Computer Applications in Power, pp. 36-39, July 1999.

[68] M. Adamiak and A. Kulshrestha, "Design and implementation of a UCA based substation control system," tech. rep., General Electric, 2002. 